WHOI-89-48

\title{
Calculation of 3-Dimensional Synthetic Seismograms on the Connection Machine
}

\author{
by \\ J.M. Allen and D.R. Burns \\ Woods Hole Oceanographic Institution \\ Woods Hole, Massachusetts 02543
}

October 1989

\section{Technical Report}

Funding provided by Office of Naval Research under

Contract Numbers: N00014-87-K-0007 and N00014-89-J-1012.

The Connection Machine operated by the Naval Research Laboratory Connection Machine Facility was used for part of this work. Another part of this work was conducted using the computational resources of the Northeast Parallel Architectures Center (NPAC) at Syracuse University, which is funded by and operates under contract to DARPA and the Air Force Systems Command, Rome Air Development Center (RADC), Griffiss Air Force Base, NY, under contract \#F306002-88-C-0031.

Reproduction in whole or in part is permitted for any purpose of the United States Government. This report should be cited as:

Woods Hole Oceanog. Inst. Tech. Rept., WHOI-89-48.

Approved for publication; distribution unlimited.

\section{Approved for Distribution:}

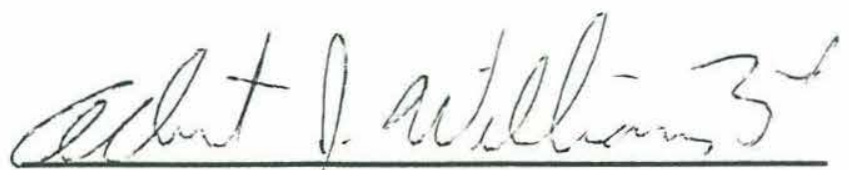

Albert J. Williams 3rd, Chairman

Department of Applied Ocean Physics and Engineering 


\section{Contents}

1 Introduction 1

1.1 The Connection Machine $\ldots \ldots \ldots \ldots \ldots \ldots \ldots \ldots \ldots$

1.2 Programming the Connection Machine . . . . . . . . . . . . . . 2

2 The Model $\quad 2$

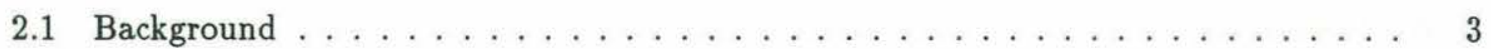

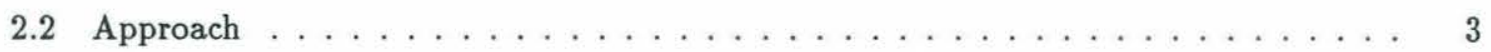

3 Running the synthetic seismogram program 5

3.1 Logging on via telnet . . . . . . . . . . . . . . . . . . 5

3.2 Defining the model input parameters . . . . . . . . . . . . . . . . 7

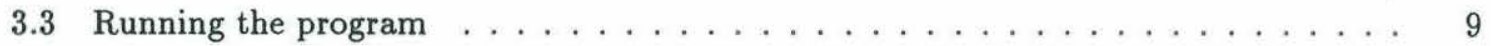

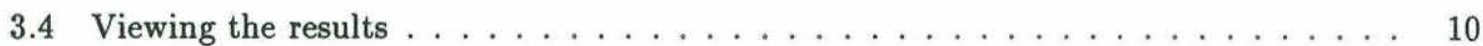

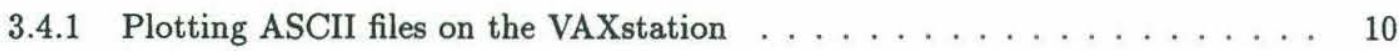

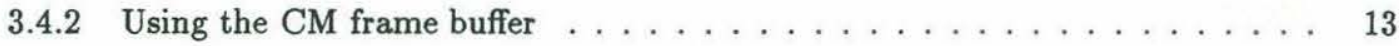

4 Guide to programming on the Connection Machine 16

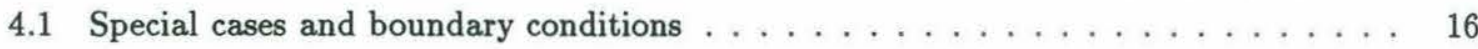

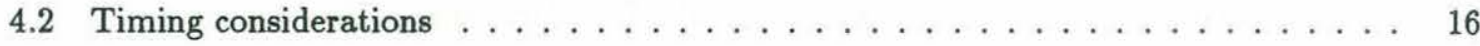

4.3 Converting serial FORTRAN to parallel $C^{*} \ldots \ldots \ldots \ldots \ldots \ldots \ldots$

4.3.1 Convert from FORTRAN to standard C . . . . . . . . . . . . . . 17

4.3.2 Determine portions of the code which could be run in parallel . . . . . . . . 17

4.3 .3 Convert from standard $\mathrm{C}$ to parallel $\mathrm{C}^{*} \ldots \ldots \ldots \ldots \ldots \ldots$

4.3 .4 Optimize the $\mathrm{C}^{*}$ code $\ldots \ldots \ldots \ldots \ldots \ldots \ldots \ldots \ldots$

4.4 File transfer via ftp . . . . . . . . . . . . . . . . 21

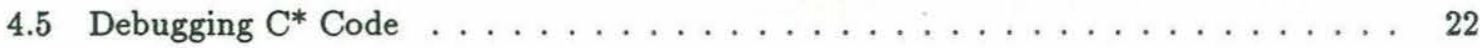

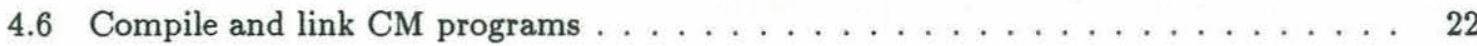

4.7 Running a $\mathrm{C}^{*}$ program on the Connection Machine . . . . . . . . . . . . . 22

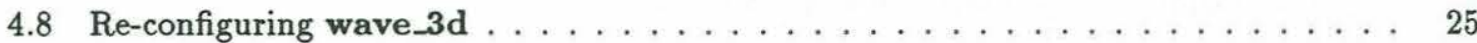


6 DataVault

$\begin{array}{lr}\text { A NRL cstar library } & 28\end{array}$

A.1 N-dimensional grid . . . . . . . . . . . . . . . . . . 28

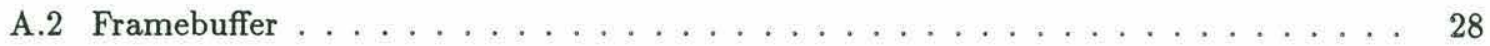

$\begin{array}{lr}\text { B cmusers group } & 28\end{array}$

$\begin{array}{ll}\text { C Structure diagram for wave_3d.cs } & 32\end{array}$

$\begin{array}{ll}\text { D Source Code } & 33\end{array}$

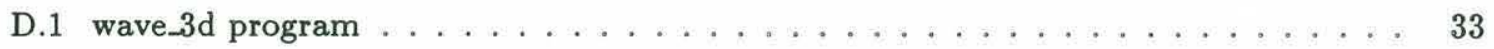

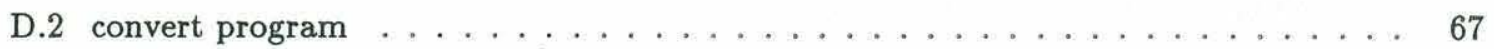

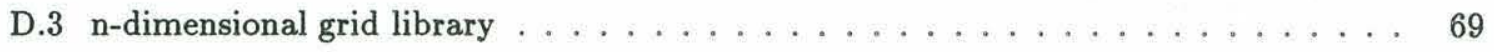

D.4 frame buffer library . . . . . . . . . . . . . . . . 75

$\begin{array}{ll}\text { References } & 79\end{array}$

\section{List of Figures}

1 Example of logging on and off the NRL and NPAC Connection Machines ..... 6

2 Sample input data file (xmodel.dat) $\ldots \ldots \ldots \ldots \ldots \ldots$

3 Example of cmattach command . . . . . . . . . . . . . . . . 11

4 Example of cmfinger and cmusers commands . . . . . . . . . . . . . 12

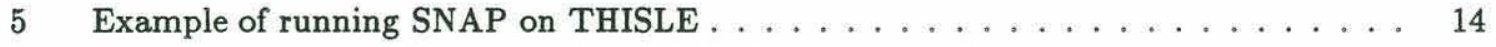

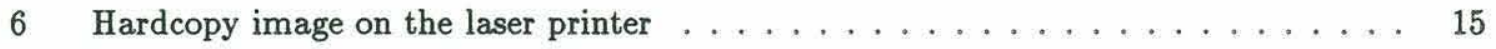

7 Declaration of domain for $3 \mathrm{D}$ synthetic seismograms . . . . . . . . . . . . 19

8 Example of serial FORTRAN vs parallel $C^{*} \ldots \ldots \ldots \ldots \ldots \ldots \ldots$

9 Example of using file transfer protocol $(\mathrm{ftp}) \ldots \ldots \ldots \ldots \ldots \ldots$

10 Compilation of wave $3 \mathrm{~d} \ldots \ldots \ldots \ldots \ldots \ldots \ldots \ldots . \ldots \ldots$

11 Segmentation of large model using DataVault . . . . . . . . . . . . . 27 


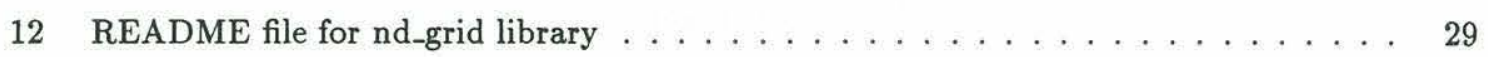

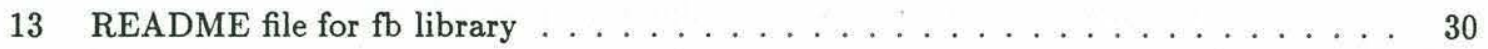




\begin{abstract}
A three dimensional, second order finite difference method was used to create synthetic seismograms for elastic wave propagation in heterogeneous media. These synthetic seismograms are used to model rough seafloor, the shallow crust, or complex structural and stratigraphic settings with strong lateral heterogeneities. The finite difference method is preferred because it allows models of any complexity to be generated and includes all multiple scattering, wave conversion and diffraction effects. The method uses a fully staggered grid as developed by Virieux (1986). Wavefront snapshots and time series output allow the scattering and focussing of different wave modes with direction to be visualized.

The extensive calculations required for realistic size models stretches the resources of serial computers like the VAX 8800 . On the Connection Machine, a massively parallel computer, the finite difference grid can be directly mapped onto the virtual processors, reducing the nested time and space loops in the serial code to a single time loop. As a result, the computation time is reduced dramatically.
\end{abstract}




\section{Introduction}

Most computers used today are based on the von Neuman (serial) architecture. Serial computers have a single central processing unit (CPU) and memory; data from memory is passed to the CPU one data element at a time and instructions are executed in sequence. These computers are termed single-instruction, single-datastream (SISD) architecture since one instruction at a time is executed on a single data item. Although the efficiency of SISD computers may be enhanced by hardware techniques such as pipelining and vector processors, the time required to complete computationally intensive programs is often prohibitive.

Parallel systems can be grouped into two broad categories: single-instruction, multiple-datastream (SIMD) and multiple-instruction, multiple-datastream (MIMD). SIMD computers are characterized by a large number of simple processors, each with its own local memory connected by a network communications system. MIMD computers typically have a smaller number of conventional processors with shared memory which execute portions of a program concurrently.

In an application such as synthetic seismograms, which requires both large volumes of data and extensive calculations, the time required to cycle all the data through one CPU places severe limitations on the size of models which can be run on a serial computer. This report documents the procedure used to implement a software system to compute 3-dimensional synthetic seismograms by the finite differences method on the Connection Machine, a SIMD computer. The advantage of parallel processing on a SIMD computer is that each node of the $3 \mathrm{D}$ model can be assigned to a single processor. Calculations are then performed simultaneously on all grid points. An operation which would normally be performed within a repetitive loop is replaced by a single operation on many processors acting in parallel.

Two dimensional synthetic seismogram models were previously coded in FORTRAN 77 to run on VAX 11/780, VAX 8800, Cyber 205 and Cray XMP-12 computers (Hunt and Stephen, 1986). A FORTRAN 77 program for a three-dimensional solution to the elastic wave equation by the method of finite differences, which runs on the VAX 8800 , was used as the basis for the parallel programs written in $\mathrm{C}^{*}$. The parallel program is called wave_3d.

\subsection{The Connection Machine}

The Connection Machine (CM) is a massively parallel computer developed by Thinking Machines, in Cambridge, MA. Massively parallel, or fine-grain computers have many simple processors, each with its own local memory. The Connection Machines used in this project are located at the Naval Research Laboratory (NRL) Connection Machine Facility and at the Northeast Parallel Architectures Center (NPAC) at Syracuse University. NRL has two CM-2 machines, one with $8 \mathrm{k}$ processors (Bambi) and one with $16 \mathrm{k}$ processors (Godzilla). NPAC also has two Connection Machines, a $32 \mathrm{k} \mathrm{CM}-1$ (CUBE) and a $32 \mathrm{k} \mathrm{CM-2} \mathrm{(SON-OF-CUBE).}$

The Connection Machine processors are divided into groups of $8 \mathrm{k}$ or $16 \mathrm{k}$ processors. A single Connection Machine can have one, two or four groups of processors. The 16k NRL CM-2 has two groups of $8 \mathrm{k}$ processors; the NPAC $32 \mathrm{k}$ CM-2 has 4 groups of $8 \mathrm{k}$ processors. Each group of processors is associated with a sequencer which interprets the instructions sent by a front-end computer. In order to use the Connection Machine system, the front-end must logically attach itself to one or more sequencers. 
Each processor on the Connection Machine model CM-2 has 64K bits (2048 words) of memory; each CM-1 processor has $4 \mathbf{k}$ bits of memory. The Connection Machine processors are connected by a network communications system so that any processor can communicate with any other processor via a routing device wired in an n-cube pattern (Hillis,1987). The CM can be configured in software for virtual to physical processor ratios that are a power of 2 , as long as there is enough memory per virtual processor to accomodate the required calculations and variables.

The memory requirements of the wave_3d program limits the maximum virtual processor ratio to 16:1. On the 16k NRL Connection Machine (Godzilla), the maximum number of virtual processors available for the 3D grid is 262,144 ( $64 \times 64 \times 64$ or 256k); on the 32k NPAC CM-2 Connection Machine (SON-OF-CUBE), the maximum number of grid points is $524,288(64 \times 64 \times 128$ or $512 \mathrm{k})$.

\subsection{Programming the Connection Machine}

The CM is connected by a high speed bus to a conventional serial computer which serves as the user-interface. Program development is accomplished on the front-end computer using the editors and compilers of the front-end processor. All of the Connection Machines used in this project support $\mathrm{C}^{*}, \mathrm{C} /$ Paris, ${ }^{*}$ Lisp and CM Fortran.

The NRL Connection Machines Bambi (8k) and Godzilla (16k) are connected to four front-end computers:

1. cmvax

(VAX 8800)

2. cmsun (SUN 4/280)

3. THINK75 (Symbolics 3675)

4. THINK40 (Symbolics 3640)

The NRL CMs are interfaced to the front-ends as follows:

$\begin{array}{ccccc}\text { Interface } & \text { cmvax } & \text { cmsun } & \text { THINK75 } & \text { THINK40 } \\ 0 & \text { Bambi } & \text { Bambi } & \text { Godzilla } & \text { Bambi } \\ 1 & \text { Godzilla } & \text { Godzilla } & & \end{array}$

The VAX front-end (cmvax) runs the ULTRIX operating system; the SUN front-end (cmsun) runs the UNIX operating system.

The two Connection Machines at NPAC (CUBE and SON-OF-CUBE) are connected to a VAX front-end (cmx) which runs ULTRIX 2.2, a 4.2BSD-based system.

The parallel program is downloaded onto the $\mathrm{CM}$ at runtime. The synthetic seismogram program, wave_3d, is written in $\mathrm{C}^{*}$, an extension of the $\mathrm{C}_{\text {programming language, developed to allow parallel }}$ execution. The wave_3d program contains both serial and parallel code.

\section{The Model}

The finite difference method is used to create synthetic seismograms for models containing lateral heterogeneity; this method is preferred because it allows models of any complexity to be generated and includes all multiple scattering, wave conversion, and diffraction effects. Most analytical modelling techniques uge assumptions, such as primary scattering (Born and Rytov approximations), 
which limit their applicability to weak heterogeneity situations. Modelling of a rough seafloor, the shallow crust, or complex structural and stratigraphic settings, however, must be able to handle strong lateral heterogeneities. In addition, the finite difference technique can be used for all ratios of scatterer size to wavelength.

\subsection{Background}

The finite difference method involves the spatial and temporal discretization of the wave equation on a regular grid which represents the model of interest. The method has been successfully applied to an extremely wide range of seismic wave propagation problems including earthquake seismology (Alterman and Karal, 1968; Frankel and Clayton, 1986; Toksoz et al., 1988), marine refraction (Stephen 1983; Dougherty and Stephen, 1988), reflection seismology (Kelly et al. 1976; Virieux, 1986; Fornberg, 1987), VSP (Stephen, 1984), and full waveform acoustic logging (Stephen et al., 1985). A number of formulations have been utilized for these applications, including second and fourth order formulations, and the pseudospectral method. Fornberg (1987) presents a comparizon of these different methods. The higher order schemes are more accurate, require fewer grid points per wavelength, and are more computationally demanding. The lower order schemes are more efficient computationally, but require more grid points per wavelength, and therefore more computer memory. The second order scheme has been fully validated against analytic methods (Stephen, 1983, 1988) and can handle complicated interfaces and boundaries. In addition, the use of fully staggered grids for displacements and stresses (Virieux, 1986; Dougherty and Stephen, 1988) improves the accuracy and stability of the formulation at no additional cost in computation or memory. The second order scheme has been successfully applied to 2-D heterogeneous media by Dougherty and Stephen (1988), and 3-D media by Etgen and Yomagida (1988). Because the primary interest is in modelling rough interface effects, even the pseudospectral method will need fine grid spacing to accurately represent such interfaces. Therefore, the memory requirement differences between the lower order methods and the pseudospectral method are not es great as for other types of applications.

\subsection{Approach}

The system of equations we wish to solve is given by:

$$
\begin{gathered}
\rho \frac{\partial^{2} u}{\partial t^{2}}=\frac{\partial \tau_{x x}}{\partial x}+\frac{\partial \tau_{x y}}{\partial y}+\frac{\partial \tau_{x z}}{\partial z} \\
\rho \frac{\partial^{2} v}{\partial t^{2}}=\frac{\partial \tau_{x y}}{\partial x}+\frac{\partial \tau_{y y}}{\partial y}+\frac{\partial \tau_{y z}}{\partial z} \\
\rho \frac{\partial^{2} W}{\partial t^{2}}=\frac{\partial \tau_{x z}}{\partial x}+\frac{\partial \tau_{y z}}{\partial y}+\frac{\partial \tau_{x z}}{\partial z} \\
\tau_{x x}=(\lambda+2 \mu) \frac{\partial u}{\partial x}+\lambda \frac{\partial v}{\partial y}+\lambda \frac{\partial w}{\partial z} \\
\tau_{y y}=\lambda \frac{\partial u}{\partial x}+(\lambda+2 \mu) \frac{\partial v}{\partial y}+\lambda \frac{\partial w}{\partial z} \\
\tau_{z z}=\lambda \frac{\partial u}{\partial x}+\lambda \frac{\partial v}{\partial y}+(\lambda+2 \mu) \frac{\partial w}{\partial z}
\end{gathered}
$$




$$
\begin{aligned}
& \tau_{x z}=\mu\left(\frac{\partial u}{\partial z}+\frac{\partial w}{\partial x}\right) \\
& \tau_{x y}=\mu\left(\frac{\partial u}{\partial y}+\frac{\partial v}{\partial x}\right) \\
& \tau_{y z}=\mu\left(\frac{\partial v}{\partial z}+\frac{\partial w}{\partial y}\right)
\end{aligned}
$$

Where $u, v, w$ represent the $x, y, z$ displacements, $\lambda$ and $\mu$ are the Lame parameters, $\rho$ is the density, $\tau_{i j}$ the stress terms, and $t$ represents time. For heterogeneous media, the Lame parameters and density all vary spatially in three dimensions. A unique staggered spatial grid can be constructed to solve this system with centered differences. This grid is a direct extension of the two dimensional grid given in Virieux (1986).

In order to avoid grid dispersion in the model, the highest frequency component to be modelled must be sampled by $10-30$ grid points per wavelength (Kelly et al., 1976; Stephen, 1983).

A dilatational or explosive point source is introduced into the grid using the method developed by Nicoletis (1981). This method computes a force distribution over a finite volume of the three dimensional grid to represent a dilatational source. The accuracy of this representation increases as the grid volume on which it is imposed is increased. The time dependency of the source takes the form of the derivative of the Gaussian distribution with any given center frequency (Kelly et al. 1976; Stephen et al., 1985).

Boundary conditions for this 3-dimensional staggered grid formulation sre fairly straight forward. The source is introduced inside the model, and the six planes which define the model limits are treated as absorbing boundaries using the telegraph equation in a zone around each of the planes (Levander, 1985; Dougherty and Stephen, 1988). Because the finite difference model is formulated for heterogeneous media, interfaces within the model do not require any specific boundary conditions to be coded. Interfaces of any complexity are handled implicitly (with the caveat that steeply dipping interfaces must be adequately sampled by the spatial grid).

The memory and computational requirements for numerical modelling in three dimensions are great. For the second order three-dimensional code which we have developed, twelve (12) real variables must be stored for each grid point in the heterogeneous section of the model (three displacement values at three time steps, plus two elestic constants and density). In order to reduce the memory requirements on the VAX, the model assumes a heterogeneous zone sandwiched between two homogeneous zones. As an example of the memory and computational requirements on a serial machine, a model containing $80 \times 80 \times 50$ grid points needs 12.5 Mbytes of central memory and the run time for 400 time steps was about 20 hours on a VAX 8800 . These requirements can be reduced by reducing the heterogeneous zone to as small a volume as needed for the models of interest or by segmenting the model into several runs.

Fortunately, computer hardware advances in parallel processing can solve this problem. On a massively parallel processing machine the finite difference grid can be directly mapped onto the processors, reducing the nested time and space loops in the serial code to a single time loop in the parallel code. As a result, the computation time is reduced dramatically. Other parallel machines are designed with fewer more powerful processors. On these types of machines, each processor might run the finite difference code for a segment of the model, and communicate displacement values at the end of each time step. Any parallel computer can greatly reduce computation time and, therefore, make three dimensional numerical modelling feasible. 


\section{Running the synthetic seismogram program}

This section describes the steps necessary to define the model, run the synthetic seismogram program and view the three-dimensional model created by the program wave-3d. Although some familiarity with the UNIX operating system is helpful, the following sections provide a complete description of the steps required.

To run program wave_3d:

1. Log on to the front-end via telnet (from the WHOI RED VAX)

2. Define the model parameters in the file xcmodel.dat

3. Run the wave_3d program on the Connection Machine

4. View the results by either:

(a) copying the ASCII output files to the VAX (via ftp) and using the SNAPSHOT program on the WHOI THISLE VAX Workstation, or

(b) running wave_3d with the frame buffer option set and viewing the results on the CM Graphic Display System (GDS) high resolution color monitor (note that there should be someone at NRL or NPAC who can physically watch the frame buffer monitor!)

\subsection{Logging on via telnet}

Access to the Connection Machine front-end computers at NRL and NPAC is via the ARPA Internet link using telnet (figure 1).

1. $\log$ on to the WHOI Red VAX

To access the Connection Machine at NRL:

RED \$ telnet 134.207.7.12 (for the VAX interface - cmvax)

RED $\$$ telnet 134.207.7.4 (for the SUN interface - cmsun)

To access the Connection Machine at NPAC:

RED_s telnet cmx.npac.syr.edu (for the VAX interface - cmx)

Note: when using telnet, you could get the following message:

RED $\$$ telnet 134.207.7.12

Trying... Attempt to connect to foreign host failed: Hetrork is unreachable RED $\$<<<$ at this point, just wait and try again later! $>>>$

2. log on to emvax, cmsun or cmx; after logging on, one of the following prompts will appear:

cmvax\% (if logged on to the VAX front-end at NRL)

cmsun\% (if logged on to the SUN front-end at NRL)

conx\% (if logged on to the VAX front-end at NPAC) 
Figure 1a. Logging on to the NRL SUN front-end (cmsun)

RED \$ telnet 134.207.7.4

Trying ... Open

Connected to 134.207.7.4.

Escape character is ' $]$ '.

SunOS URIX (cmsun)

login: allen

Passpord: $<<<$ type password here $>>>$

Last login: Thu hag 31 08:37:32 Irom RED. UHOI.EDU

Sunos Release 4.0 (CKSUH) \#64: Tue hng 22 12:45:06 EDT 1989

ERL Connection Machine Facility - Su 4/280

$<<<$ system messages here $>>>$

TERH $=($ vt100) $<<<$ hit return here $>>>$

allen . cmono\% lo

Connection closed to 134.207 .7 .4

RED_\$ $\ll<$ back to DCL prompt on RED VAX $\gg>$

Figure 1b. Logging on to the NRI VAX front-end (cmvax). RED \$ telnet 134.207.7.12

Trying... Open

Connected to 134.207.7.12.

Escape charaeter i.s' " J'.

convax login: allen

Passzord: $<<<$ type password here $>>$

Last login: Tue lug 8 11:31:48 Irom 128.128.16.78

U1trix-32 v3.0 (Rev 64) System \#7: Sat Jul 8 17:34:53 EDT 1989

vRL Connection Machine Facility - VLX Be00 « eyttem messages here $\gg>$

TEAH $=($ vt100 $)<<<$ hit return here $>>>$

allen. curar\% lo

Connection closed to 134.207 .7 .12

AED_\$ << beck to DCL prompt on $\mathrm{AED} V M X>>$

Figure 1c. Logging on to the NPAC VAX front-end $(\operatorname{cmx})$.

REDS telnet cmx.npac.syr.edu

Trying...

Open

Connected to cmx.npac. ayx.edn.

Eacape character is '-J'.

ULtrix-32 v3.0 (Rev 64) (Cmx.mpac.egr.edu)

login: jallen

Passeord: $<<<$ type password here $>>>$

Last login: The Sep 6 09:17:39 1rom RED.UBOI.EDO

V1trix-32 V3.0 (Bar 64) Syatem i1: Hon Jol 17 11:24:69 EDT 1989

« ejeteh meanages hare $\gg>$

Tue Sep 5 09:17:40 EDT 1089

$\cos \%$ Io

Connection cloged to cmx.hpac.agr.edu

RED_\$ << back to DGL prompt on RED VAX $\gg>$

Figure 1: Example of logging on and off the NRL and NPAC Connection Machines 


\subsection{Defining the model input parameters}

Input to program wave_3d is via the ASCII file xmodel.dat. The contents of xmodel.dat are as follows:

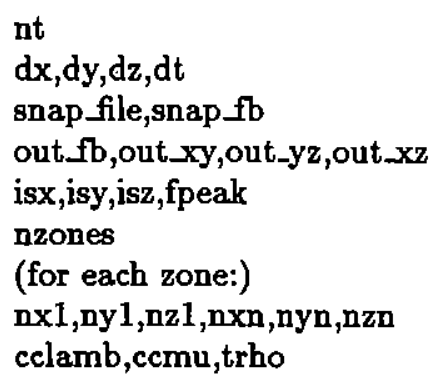

where:

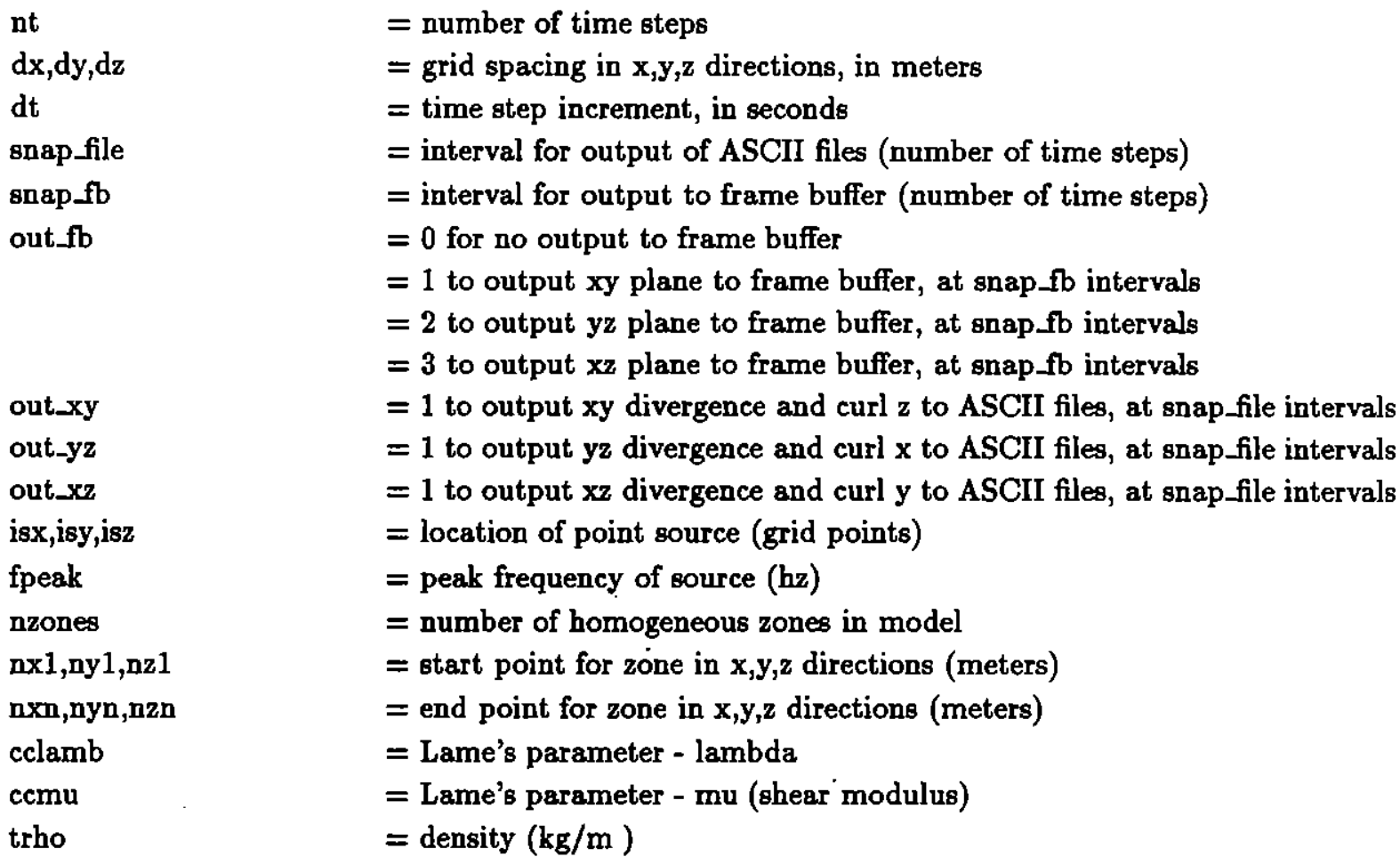

Figure 2a shows a sample input data file for the model illustrated in figure $2 b$. Note that when defining the boundaries of the model areas, the indices ( $\mathrm{nx} 1, \mathrm{ny1}, \mathrm{nz} 1, \mathrm{nxn}, \mathrm{nyn}, \mathrm{nzn})$ should range from 0 to 63 , rather than from 1 to 64 .

Use the vi editor on the front-end to edit this file or use the edt editor on the WHOI RED VAX and ftp the xmodel.dat file to the CM front-end (see section 3.4 for directions on transferring files via $\mathrm{ftp}$ ). 
Figure 2a. Sample file xmodel.dat for beterogeneous model.

This file describes the parameters for the model in figure $2 \mathrm{~b}$. The $3 \mathrm{D}$ grid is $64 \times 64 \times 64$; the point source is located at $(10,31,10)$; there will be no output to the frame buffer; ASCII files created will be: div_xy.dat, curl.z.dat, div_xz.dat, curl_y.dat, div_yz.dat, and curl_x.dat. The model will be calculated for 250 time steps and data will be output to the files at every 50 time steps.

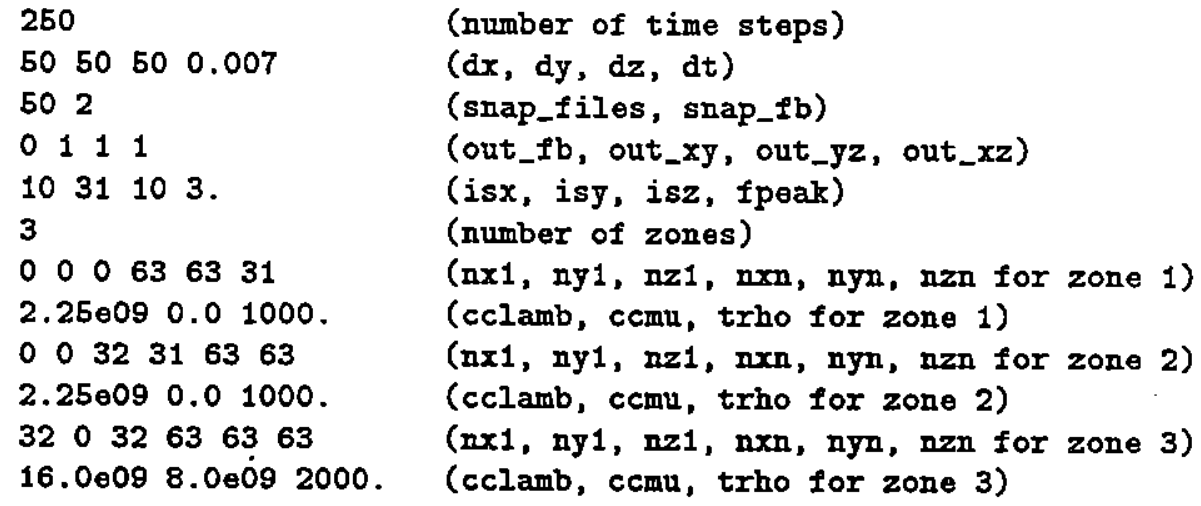

Figure 2b. Three dimensional model calculated in the examples presented in this technical report.

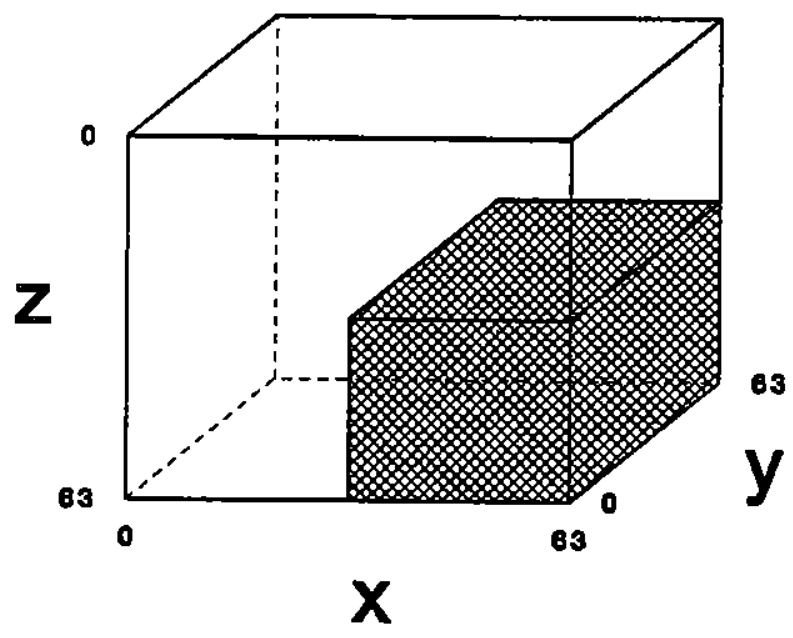

Figure 2: Sample input data file (xmodel.dat) 


\subsection{Running the program}

Program wave $3 d$ is configured for a model with dimensions $64 \times 64 \times 64$; this model requires $262144(256 \mathrm{k})$ virtual processors. The program has been optimized to run at a maximum virtual processor ratio of 16:1. This means that a minimum of $16 \mathrm{k}$ processors is necessary to run this model. When running the program at NRL, all 16k processors of the Godzilla Connection Machine must be allocated. On the 32k NPAC CM, only two of the four sequencers on SON-OF-CUBE are needed to run the program. The program can also be configured to run a model with dimensions $64 \times 128 \times 64$ on the NPAC 32k CM if all four sequencers on SON-OF-CUBE are used (see section 4.8).

To run the wave_3d program (figure 3):

1) Determine if the required sequencers on the $\mathrm{CM}$ are available using the cmfinger and cmuser commands (figure 4); you will not be allowed to attach to the CM if it is busy

2a) on the NRL CM, type the following command:

cmvax\% cmattach -b 0.95 -p 16k -v 256k -i1 wave_3d > wave_3d.out \&

$2 \mathrm{~b})$ on the NPAC CM, use this command:

cmx\% cmattach -b 0.95 -p 16k -v 256k -C S wave_3d > wave_3d.out \&

The file wave_3d.out will contain the runtime diagnostic messages and the timing information after program completion. If the CM is busy and you wish to attach and 'wait for resources', add the $-w$ flag to the command line:

cmvar\% cmattach -b 0.95 -p 16k -v 256k -w -il wave_3d > wave_3d.out \&

or

cmx\% cmattach -b 0.95 -p 16k -v 256k - w -C S wave_3d > wave_3d.out \&

This option will cause the cmattach program to wait (possibly forever) for the desired resources (namely both sequencers of Godzilla or two sequencers of SON-OF-CUBE) to be freed. Note that this is a fairly primitive implementation and simply causes the job to be resubmitted once every minute. If the sequencers are freed up during the minute that the job is waiting, they can be attached by anyone else. If you are in a hurry, it is safer, and usually faster, to use cmfinger to keep an eye on which sequencers are free and then resubmit the cmattach command. The c-shell has a history command that is useful for this:

cmx\% !cma (the most recent command beginning with cma will be executed)

The output file should have a unique name on the NRL CM. If a file with that name exists already, the following message will appear:

cmson\% cmattach -b 0.95 -p $16 k$-v 256k -i1 wave_3d > wave_3d.out \&

[1] 13999

vave.3d.out file existe

cmsan\%

Either choose a different name for the output file on the command line, or remove the current file and re-submit the attach command: 


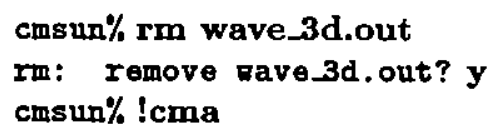

On the NPAC CM, the file will be overwritten if it already exists.

To have diagnostic messages appear on the terminal screen rather than in a file, simply omit the redirection (> vave.3d.out):

cmsun\% cmattach -b 0.95 -p $16 \mathrm{k}$-v $256 \mathrm{k}$-i1 wave_3d \&

or

cmx\% cmattach -b 0.95 -p $16 k-v 256 k-C$ S wave_3d \&

\subsection{Viewing the results}

In order to view the results of the 3-dimensional synthetic seismogram program, 2-dimensional 'slices' through the center of the model may be:

1. output as ASCH files and transferred to a VAXstation for plotting, or

2. output to the Connection Machine frame buffer during runtime.

\subsubsection{Plotting ASCII files on the VAXstation}

Program wave_3d writes two ASCII files (one containing divergence and the other containing curl) for each 2-dimensional plane selected, every time step interval. The filenames are determined by the 2-dimensional plane and the time step:

$$
\begin{aligned}
& \frac{\text { output option }}{\text { out_xy }} \frac{\text { files }}{\text { divxy_N.dat }} \text { xy plane at } z=z \text {-dimension/2-1 } \\
& \text { curlz_N.dat } \\
& \text { out_yz divyz_N.dat } y z \text { plane at } x=x \text {-dimension } / 2-1 \\
& \text { out_xz divxz_N.dat } x z \text { plane at } y=y \text {-dimension } / 2-1 \\
& \text { curly_N.dat }
\end{aligned}
$$

where $\mathrm{N}$ is the time step.

The procedure for plotting ASCII files of divergence and curl on the VAXstation is as follows:

1. Transfer the ASCII files from the CM front-end to the RED VAX using ftp:

RED_s ftp 134.207.7.12 (refer to section 4.4)

username: allen

passnord: <<type password here $>>$

* get divxz_200.dat (to transfer divxz_200.dat from cmvax to RED)

* get curly 200.dat (to transfer curly_200.dat from cmvax to RED)

* ex (to exit ftp) 

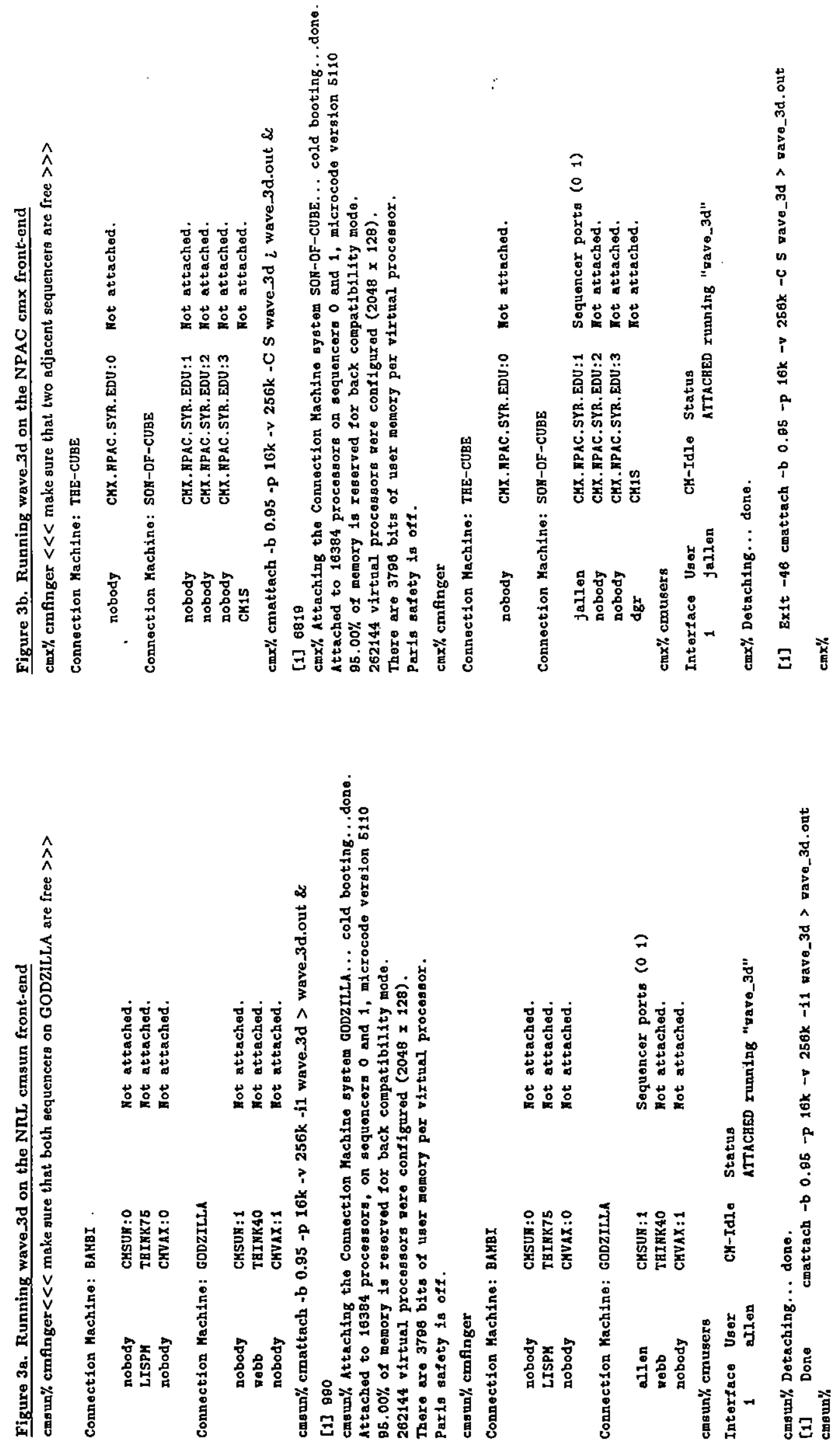
Figure 4a-Example of cmfinger and cmusers commands on the NRL cmvax front-end cmvax $\%$ cmfinger

Connection Machine: BAKBI

$\begin{array}{lll}\text { nobody } & \text { CKSUH:0 } & \text { Not attached. } \\ & \text { THIKK75 } & \text { Hot attached. } \\ \text { ahitcomb } & \text { CKVAZ:0 } & \text { Sequencer ports (O) }\end{array}$

Connection Machine: GODzILIA

$\begin{array}{lll}\text { ancona } & \text { CKSUN :1 } & \text { Sequencer ports (1) } \\ \text { abb } & \text { THIKK40 } & \text { Hot attached. } \\ \text { jenninge } & \text { CKVAX:1 } & \text { Hot attached. }\end{array}$

cmvax\% cmusers

$\begin{array}{clcl}\text { Interface } & \text { Uber } & \text { CH-Idle } & \text { Status } \\ 0 & \text { vhiteomb } & & \text { ATTACEED ronning "starlisp" } \\ 1 & \text { jennings } & : 03 & \text { LTTACEED running "cmattach" } \\ \text { cmvax\% } & & & \end{array}$

Figure $4 b$ - Example of cmfinger and cmusers commands on the NRL cmvax front-end cusur\% cmfinger

Connection Machine: BAkBI

$\begin{array}{lll}\text { tedrards } & \text { CHSUD:0 } & \text { Sequencer ports (1) } \\ & \text { THIKK7B } & \text { Not attached. } \\ \text { mandeIbe } & \text { CHVAX:0 } & \text { Sequencer porte (0) }\end{array}$

Connection Wachine: GODzILLA

$\begin{array}{lll}\text { Rlien } & \text { CHSOI: } 1 & \text { Sequencer porta }(01) \\ \text { nebb } & \text { THIRK40 } & \text { Iot attached. } \\ \text { nobody } & \text { CHVAX:1 } & \text { Iot attached. }\end{array}$

cmstu\% cmusers

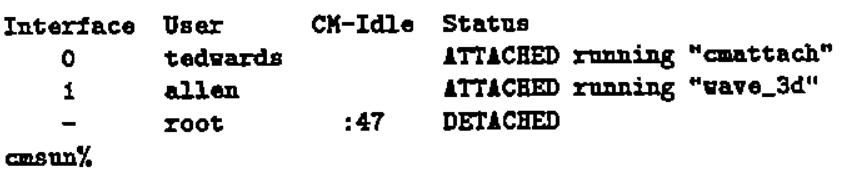

Figure 4c - Example of cmfinger and cmusers commands on the NPAC cmx front-end conx\% cmfinger

Connection Machine: THE-COBE

nobody CLI. IPAC.SY.EDU:0 Iot attached.

Connection Machine: SOF-OF-CUBE

nobody CKX.IPLC.SYR.EDU:1 Dot attuched.

root CLT.IPAC.SYR.EDO:2 Sequencer ports (3)

nobody CKX.IPAC.SYR.EDU:3 Iot attached.

CH1S Iot attached.

cmr\% cmusers

$\begin{array}{llll}\text { Interface } & \text { Joer } & \text { CH-Idle } & \text { Stutas } \\ 2 & \text { root } & 1: 52 & \text { ITfHCHED ranning " } 1 \text { " } \\ \text { cmr\% } & & \end{array}$

$\operatorname{com} \%$

Figure 4: Example of cmfinger and cmusers commands 
RED_\$

2. On the RED VAX, convert the ASCII output files to binary format using the convert program:

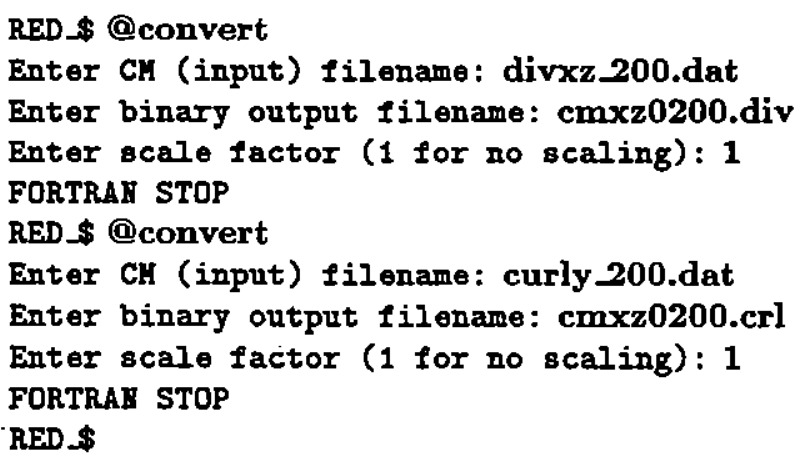

3. Log onto the VAX Workstation THISLE (THISLE is on the VAX cluster so the default data directory RNR2:[FIND.JMA1] can be accessed from either RED or THISLE).

4. On THISLE, run snap_fix to enlarge the images, if desired (this example will enlarge a $64 \times 64$ image to $256 \times 256$ ):

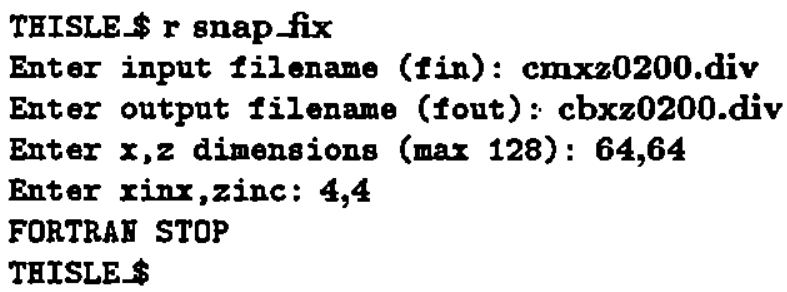

5. On THISLE, execute the command SNAP to send the image to the workstation screen and/or create an image file. The example in figure 5 creates an image file named cbxz0200.img.

6. To get a hardcopy of the image file(s) created by SNAP, log back onto the RED VAX and run the command file ing. Output is to the Imagen laser printer (figure 6):

RED \$ @img cbxz0200.img

\subsubsection{Using the CM frame buffer}

The CM graphics display aystem (GDS) consists of a frame buffer and a high-resolution color monitor (19 inch, $1280 \times 1024$ pixels), which combine to display images computed on the CM-2. The GDS can be programmed to display any 2-dimensional grid of virtual processors.

If the variable out_fb is set to 1,2 or 3 in the file xmodel.dat, images will appear on the default frame buffer at NRL or NPAC in 'real-time' (this is obviously only useful if there is someone at NRL or NPAC who is watching the monitor!).

At NRL, the four frame buffer locations are as follows: 


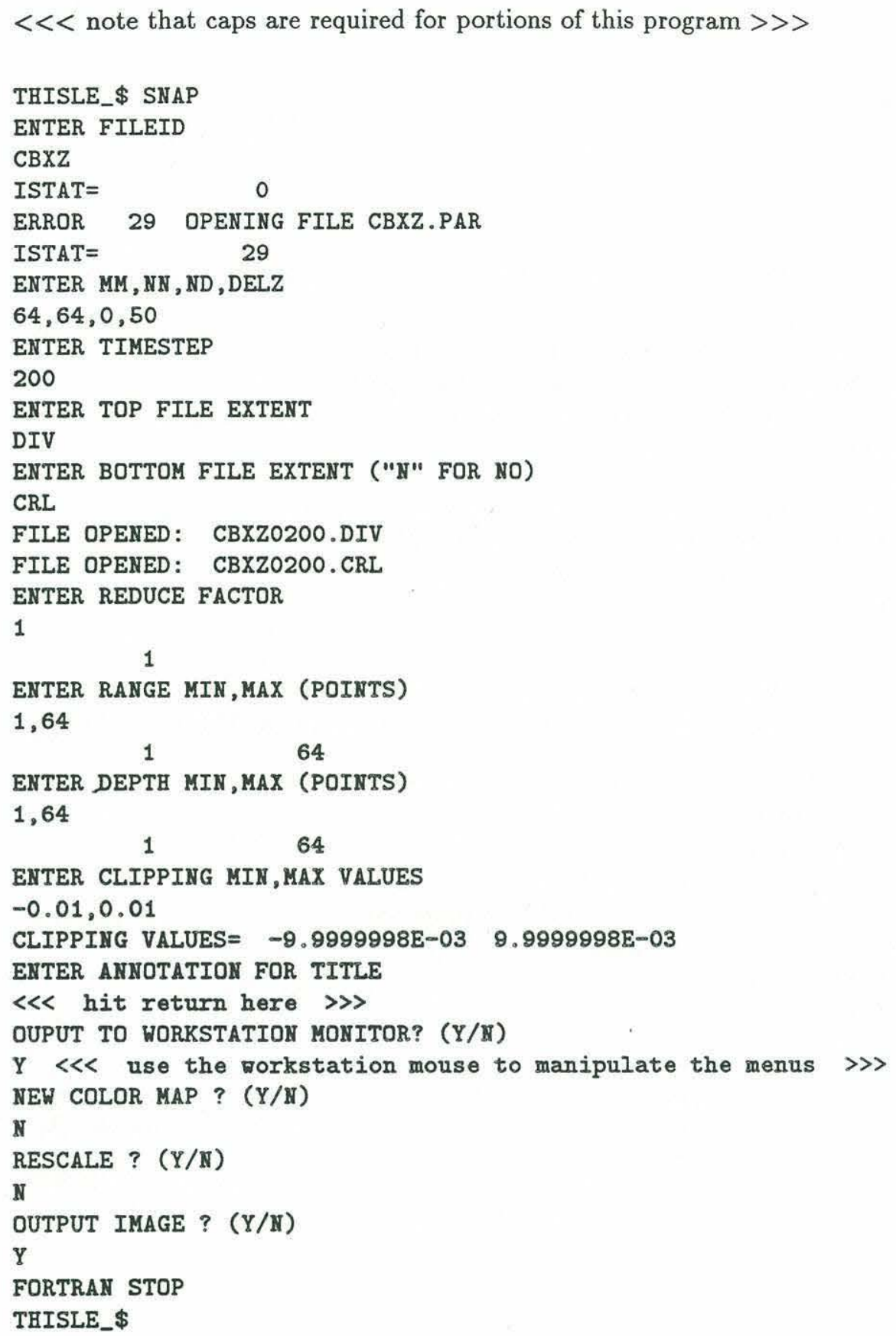

Figure 5: Example of running SNAP on THISLE 


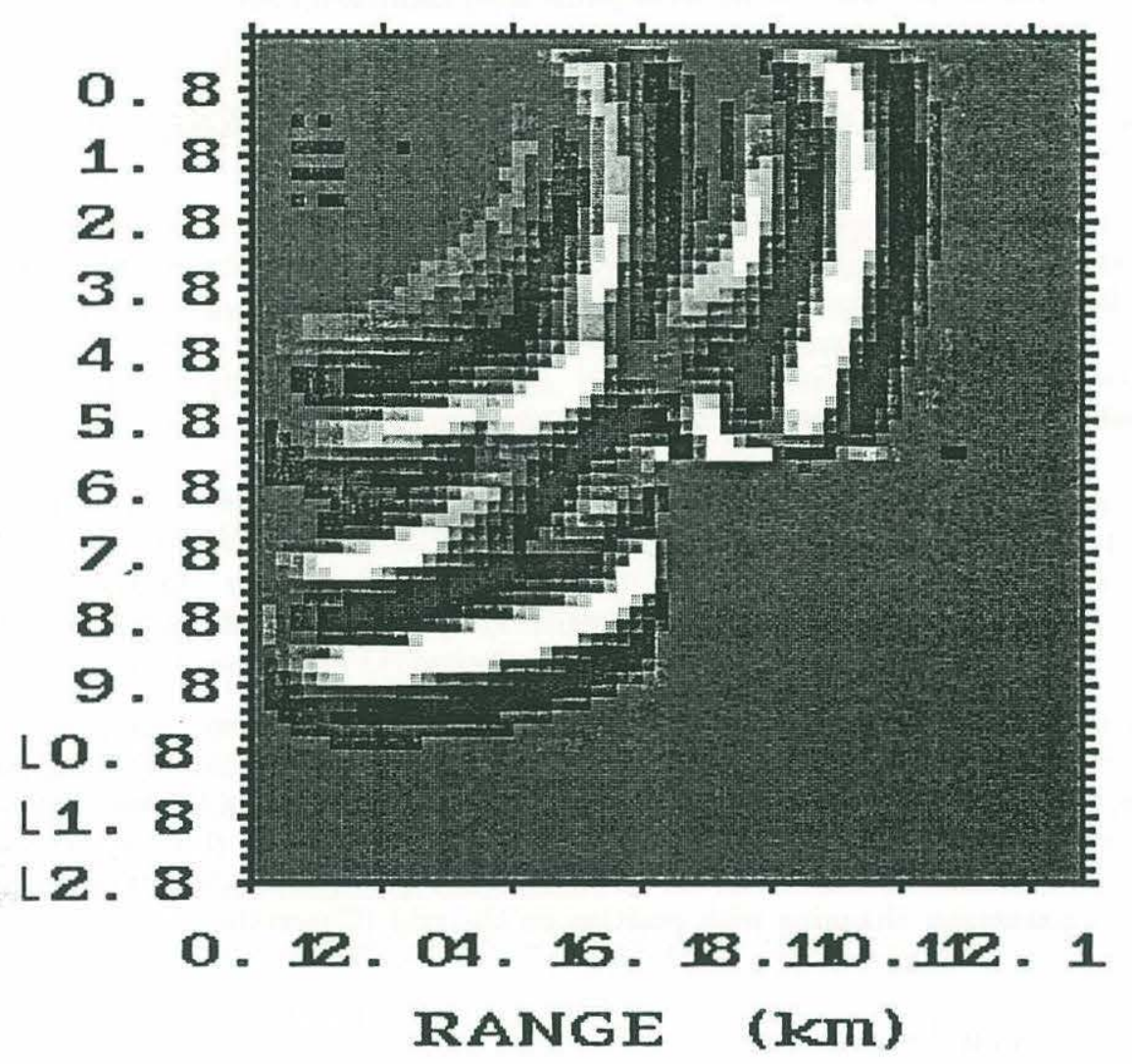

Section along the $\mathrm{x}-\mathrm{z}$ plane at time step 200 of 3-dimensional synthetic seismogram calculated with program wave_3d. The model calculated is shown in figure 2. Image displayed on microVAX workstation using program SNAPSHOT.

Figure 6: Hardcopy image on the laser printer 


$\begin{array}{lccll}\text { CM } & \text { interface } & \text { sequencer } & & \text { FB location } \\ \text { Bambi } & 0 & & 0 & \\ \text { Bambi } & 0 & 1 & \text { Ander room } \\ \text { Godzilla } & 1 & 0 & \text { Room with coffee maker/fridge } \\ \text { Godzilla } & 1 & 1 & \text { Shirron's office }\end{array}$

At NPAC, the frame buffers are attached to sequencers 0 and 2 of SON-OF-CUBE.

\section{Guide to programming on the Connection Machine}

The first step in writing a program to run on the Connection Machine is to learn to think in parallel terms. "Learning to write programs for parallel machines requires thinking in ways that are quite different from those demanded by sequential computers." (Hillis, 1987)

\subsection{Special cases and boundary conditions}

The large number of processors in the Connection Machine are controlled by the front-end computer, which sends a single instruction to all the processors simultaneously. For every instruction, each processor must either execute or not execute. Special cases and boundary conditions are one weakness of parallel processing. Algorithms which contain special cages should be redesigned, if possible.

For example, consider the calculation of boundary conditions. On a serial machine, the interior of the model would be calculated with one equation and the edges of the model with another. On the Connection Machine, however, it is most efficient for every processor to perform the same equation simultaneously. One solution is to add a damping term to the wave equation to eliminate unwanted reflections from the edges of the model. Then the same equation can be used for every processor with only the damping parameter changing with position on the grid (Charrette, 1987).

\subsection{Timing considerations}

Another power of programming on a massively parallel computer such as the Connection Machine is that increasing the size of the model (within the hardware limits of the computer) will not change the time required to perform the parallel calculations, although serial operations such as initialization will take longer. For example, wave_3d, the 3D synthetic seismogram program, has three main sections:

1. code to read model parameters and perform parallel initialization

2. code to perform parallel calculations within a serial time loop

3. serial code for output of results

It is difficult to make an accurate comparison of runtimes on the CM and runtimes on the VAX 8800. The timing facility on the CM gives real time and CM time (time spent in parallel mode) 
while the VAX timing facility gives real time and CPU time. Comparison of real times is not entirely valid because of multi-tasking on the computers. In this report, we are comparing real time on the Connection Machine with CPU time on the VAX; the performance of the Connection Machine is actually under-estimated.

A 3D model with dimensions $64 \times 128 \times 64$ and 200 time steps took 0.5 seconds (real time) for initialization and 1253.6 seconds (real time) total on the $32 \mathrm{k}$ Connection Machine at NPAC.

A 3D model with dimensions $80 \times 80 \times 50$ and 200 time steps took 400 minutes (CPU time) on the VAX 8800 . The following table summarizes the timing calculations:

\begin{tabular}{lll} 
Computer & Number of grid points & seconds / time step \\
\cline { 2 - 2 } CM-2 (32k) & $524288(64 \times 128 \times 64)$ & \\
VAX 8800 & $320000(80 \times 80 \times 50)$ & 120 (CPU time)
\end{tabular}

The Connection Machine is approximately twenty times faster than the VAX for a model with $60 \%$ more points. Although models computed on the Connection Machine are constrained by the number of virtual processors available, it may be possible to compute larger models using the DataVault, a high speed mass storage device attached to the Connection Machine (see section 6.0).

\subsection{Converting serial FORTRAN to parallel $\mathrm{C}^{*}$}

This section outlines some special features of $\mathrm{C}^{*}$ and some guidelines to assist a programmer in developing and modifying $\mathrm{C}^{*}$ code for the Connection Machine.

The original 3-dimensional synthetic seismogram program was written in FORTRAN and developed to run on the VAX, a serial computer. The conversion of the serial FORTRAN code to parallel $\mathrm{C}^{*}$ code was accomplished in four basic steps:

1. convert from FORTRAN to standard C

2. determine portions of the code which could be run in parallel

3. convert from standard $\mathrm{C}$ to parallel $\mathrm{C}^{*}$

4. optimize the $\mathrm{C}^{*}$ code

\subsubsection{Convert from FORTRAN to standard C}

One of the most common coding errors in converting from FORTRAN to $\mathrm{C}$ is in indexing. FORTRAN indexes from 1 to $\mathrm{N}$ while $\mathrm{C}$ indexes from 0 to $\mathrm{N}-1$. For example, a source located at the center of a $64 \times 64 \times 64$ model would have indices $(32,32,32)$ in FORTRAN while the source located at the center of the same model would have indices $(31,31,31)$ in $\mathrm{C}$.

\subsubsection{Determine portions of the code which could be run in parallel}

The $\mathrm{C}^{*}$ programming language was designed specifically for the Connection Machine. $\mathrm{C}^{*}$ is an extension of the $\mathrm{C}$ programming language that incorporates some of the object-oriented features of $\mathrm{C}++$ to enable parallel processing. 
The $\mathrm{C}^{*}$ language encourages the programmer to think in terms of groups of related quantities, similar to the struct concept in standard $\mathrm{C}$. In $\mathrm{C}^{*}$, the keyword domain extends the struct concept by allowing functions, as well as data objects, to be associated members of a group. Domains are based on the class concept from $\mathrm{C}++$. Once a domain is defined and member space is allocated, each instance of the domain can be thought of as having its own virtual processor. A program can contain both serial and parallel code; code is parallel only within a member function of a domain or within a selection statement which has activated a domain. Otherwise, the parallel code appears just like serial code.

Parallel variables are the default when declared within a member function and within a selection statement and can be explicitly declared with the poly keyword within serial code. Serial variables require the keyword mono if declared within a parallel context.

Many of the computations in the original synthetic seismogram program involved large, 3dimensional FORTRAN do loops where the same computation was performed for each node of the 3-dimensional model. These computations are relatively easy to think of in a parallel sense. Instead of processing each node of the model in sequence, all nodes are processed in parallel by placing the same code on each of the parallel processors. The first step is to allocate a virtual processor for each grid point using the nd_grid library routine make-grid (see Appendix A). A domain with 23 common variables and 20 parallel functions was defined for the 3D model (Figure 7). Once the parallel domain is defined, operations which require a 3-dimensional do loop in FORTRAN are reduced to a single operation in $\mathrm{C}^{*}$ (Figure 8 ).

\subsubsection{Convert from standard $C$ to parallel $C^{*}$}

The following $\mathrm{C}^{*}$ header files are available for parallel $\mathrm{C}^{*}$ programs:

$\begin{array}{ll}\text { <stdio.hs> } & \text { standard i/o library header file } \\ \text { <math.hs> } & \text { math routines header file } \\ \text { <nd_grid.hs> } & \text { header for n-dimensional NEWS routines } \\ \text { <fb.hs> } & \text { header for frame buffer routines } \\ \text { <cm/cmtimer.hs> } & \text { CM timer functions } \\ \text { <cm/cmfs.hs> } & \text { CM file structure (DataVault) functions } \\ \text { <cm/cmfile.hs> } & \text { DataVault functions }\end{array}$

\subsubsection{Optimize the $C^{*}$ code}

Each processor on the CM-2 has 64k bits of memory and this was found to be the major constraint on the maximum size model that could be run. Techniques used to decrease the amount of memory required by the program and maximize model size include:

1. re-compute values rather than store them in memory

2. break up large functions into smaller functions in order to reduce the amount of memory required

There is a 'bug' in the C* compiler which causes any floating point constant which is not implicitly appended with an $f$ to be double precision. This causes any calculations using that constant to be 


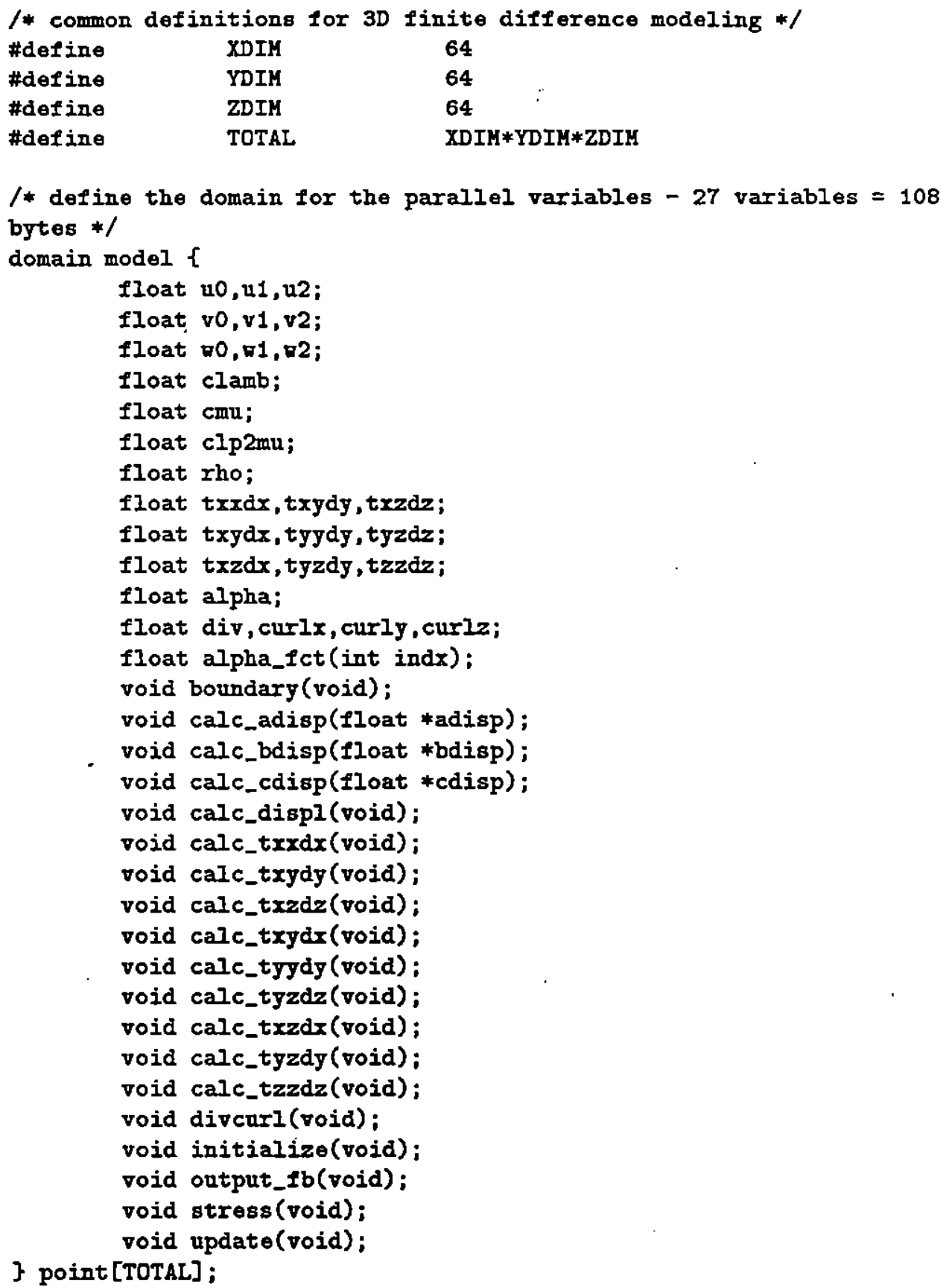

Figure 7: Declaration of domain for 3D synthetic seigmograms 


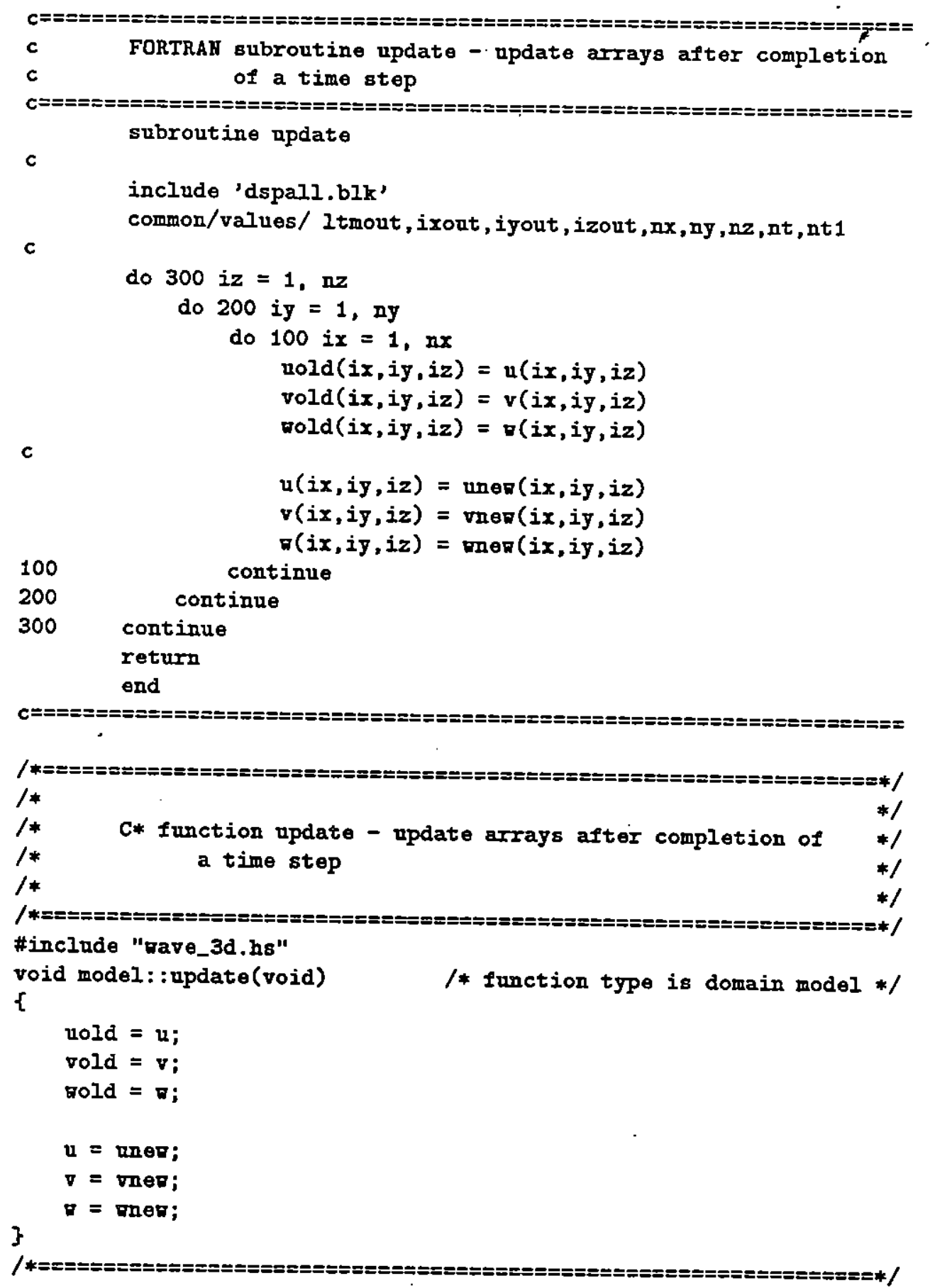

Figure 8: Example of serial FORTRAN vs parallel $C^{*}$ 
done in double precision, thus wasting valuable memory resources. To avoid this problem, append an $f$ to all floating point constants in the $\mathrm{C}^{*}$ program, for example:

\#define PI 3.141581 (instead of \#define PI 3.14159)

alpha $=\sin (((\mathrm{ir}-($ HI $-\mathrm{HTEL})) /($ HTEL $/ 2.01)) * \mathrm{PI} / 2.01) *(0.11) ;$

To detect lines containing values which would cause double precision computations, use the -w flag with the compile command:

cmx\% cs -O -w -o progname progname.cs -lnrlcstar \&

There is a timing facility in $\mathrm{C}^{*}$ for accumulating and reporting elapsed real time and $\mathrm{CM}$ time. To use the timing facility, include the header file:

\#include <cm/cmtimer.hs $>$

The timer functions used in the current program are:

CM_start_timer(1) begin accumulating timing information

CM_stop_timer(1) stop accumulating and print timing information

CM_reset_timer() reset the clock

These functions were used to time the initialization stage of the program and the total run-time. The timer functions can be implemented as $\mathrm{C}$ preprocessor macros as follows:

\#define TIKER_OI CH_start_timer(1)

\#define TIMER_RESET CM_reset_timer()

\#define TIMER_OFF CK_stop_timer(1)

\subsection{File transfer via ftp}

The internet connection to NRL is very slow. For this reason, most of the program coding and editing was done on the Red VAX at WHOI. Programs were then transferred to the CM front-end over the network using file transfer protocol (FTP), illustrated in Figure 9.

1. ftp to connect to the CM front-end

RED $\$$ ftp 134.207.7.12 $<<<$ to connect to the NRL VAX $>>>$

RED $\$$ ftp 134.207.7.4 $<<<$ to connect to the NRL SUN $>>>$

RED \$ ftp cmx.npac.syr.edu $<<<$ to connect to the NPAC VAX $>>>$

cmx\% ftp red.whoi.edu $<<<$ to connect to the WHOI RED VAX $>>>$

2. cd to the desired directory if files are in a directory other than HOME

3. use get to copy files FROM the CM front-end, use put to copy files TO the CM front-end 
4. ex to exit ftp from the WHOI VAX, (bye to exit from the UNIX front-ends)

\subsection{Debugging C* Code}

Debugging the parallel portions of a $\mathrm{C}^{*}$ program can be difficult. It is generally best to start with a simple model and print out intermediate values for selected processors. If a printf statement is used within a parallel context, it must be immediately followed with:

fflush(stdout);

This will ensure that all buffered data is sent to the output stream before parallel commands are executed.

Section 5.3.3 of the Connection Machine System C* User's Guide explains how to run C* programs with the $d b x$ debugger.

\subsection{Compile and link CM programs}

Source code to be run on the CM should have the file extent .cs and header files should have the extent .hs. Figure 10 illustrates compilation of wave_3d on the NRL and NPAC front-end computers.

To compile and link a $\mathrm{C}^{*}$ program to run on the NRL Connection Machine (the NRL C* library is installed as nrlcstar):

cmvax\% cs -O -w -O progname progname.cs -lnrlcstar \&

where:

$-\mathrm{O}$

$-\mathrm{w}$

-o progname

instead of a.out

progname.cs

-lnrlcstar

$-\mathrm{lcmfb}$

\& sets the flag for the optimizer

causes double precision calculations to be flagged

causes the executable file to be named progname

is the $\mathrm{C}^{*}$ source code file

links the program with the NRL C* library

links the program with the frame buffer library

the UNIX command for running in the background

To compile and link a C* program to run on the NPAC Connection Machine (the NRL C* library routines are local):

cmx\% cs - $\mathrm{O}-\mathrm{w}$-o progname progname.cs nd_grid.o fb.o \&

\subsection{Running a $\mathrm{C}^{*}$ program on the Connection Machine}

To run a program on the NRL Connection Machine: 


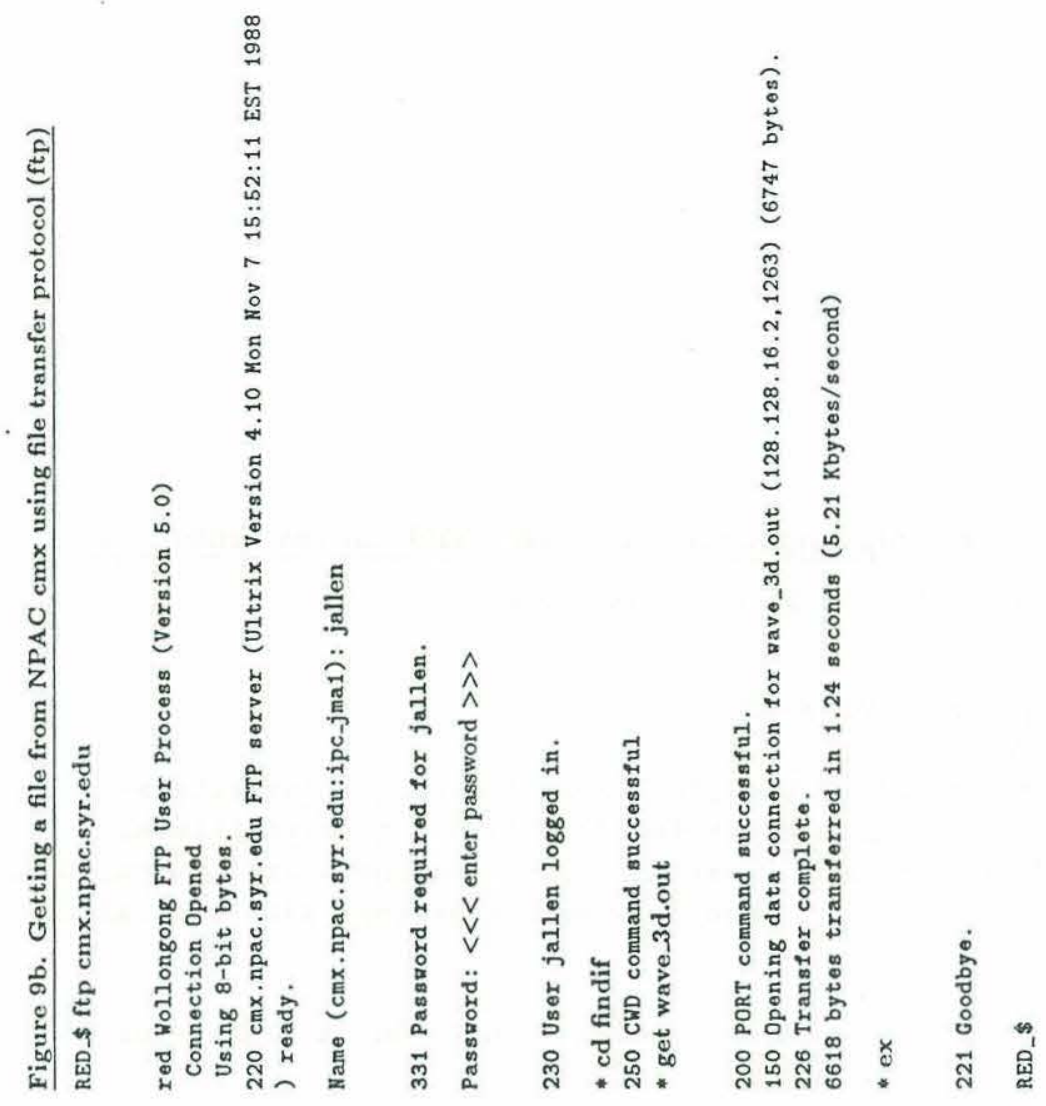

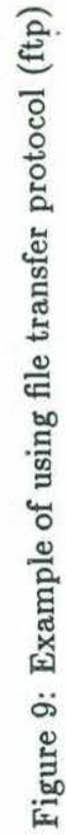
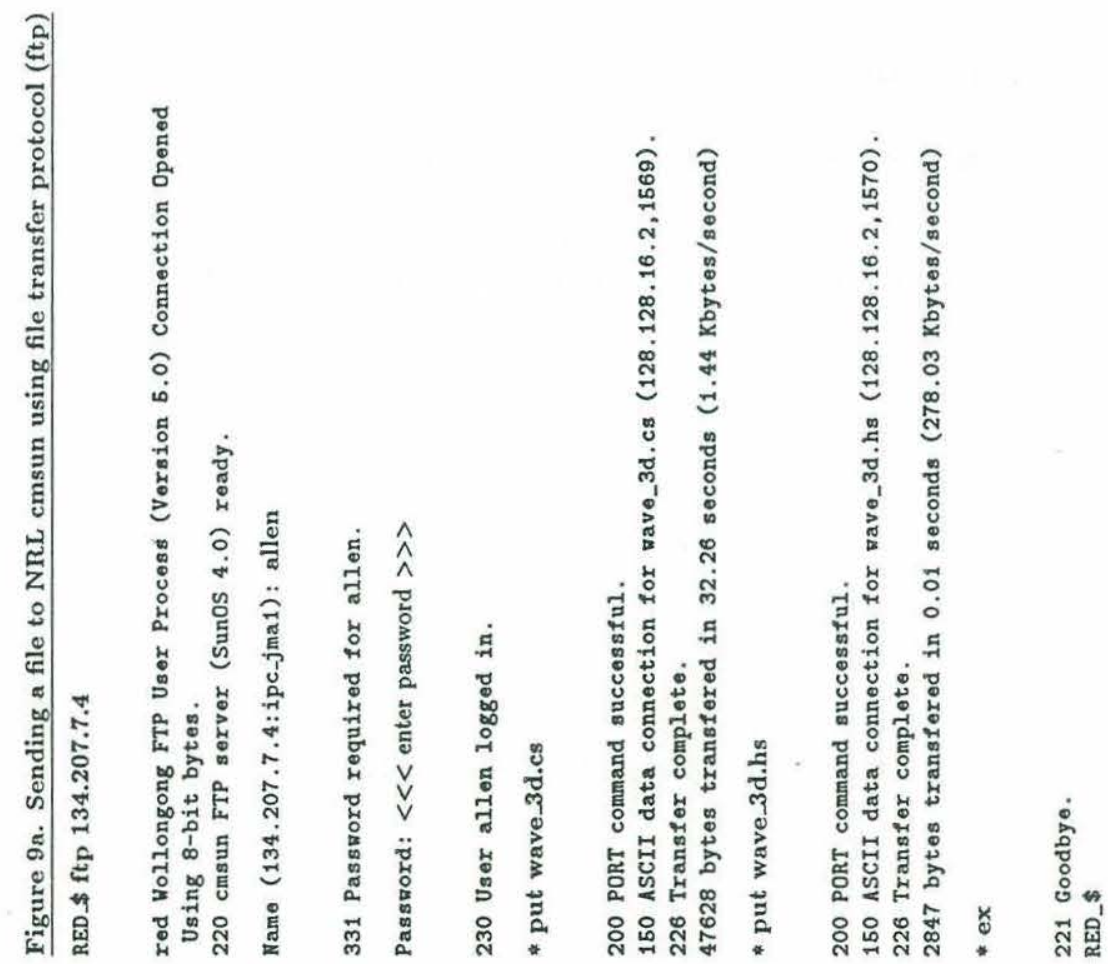
Figure 10a - Example of compiling wave_3d on the NRL cmsun front-end cmsun\% cs -O -w -o wave_3d wave_3d.cs -Inrlcstar \&

[1] 18467

wave_3d.cs : C*-compiling $\Rightarrow$ wave_3d..c

C* Compiler Version 5.0.21

"wave_3d.cs", line 98: warning: Underflow from floating-point literal 0.0.

"wave_3d.cs", line 98: warning: Underflow from floating-point literal 0.0.

"wave_3d.cs", line 100: warning: Underflow from floating-point literal 0.0.

"wave_3d.cs", line 977: varning: Underflow from floating-point literal 0.0.

wave_3d.cs: C-compiling wave_3d..c

Linking

[1] Done cs -0 - 0 -0 vave_3d wave_3d.cs - Inrlcstar

cmsun\%

Figure 10b - Example of compiling wave.3d on the NPAC cmx front-end

cmx\% cs -O -w -o wave_3d wave_3d.cs nd_grid.o fb.o \&

[1] 5996

cmx\% wave_3d.cs: C*-compiling $\Rightarrow$ vave_3d.,c

C* Compiler Version 5.0

wave_3d.cs: C-compiling wave_3d...c

Linking

[1] Done cs $-0-$ - -0 mave_3d wave_3d.cs nd_grid.o fb.o

$\operatorname{cm} \times \%$

Figure 10: Compilation of wave $\_3 \mathrm{~d}$ 
cmsun\% cmattach -b 0.95 -p nprocs - v nvprocs - $\mathrm{i}$ interface progname > output_file \&

To run a program on the NPAC Connection Machine:

cmx\% cmattach -b 0.95 -v nvprocs -p nprocs -C CM_name progname > outputffile \&

where:

$-\mathrm{b} 0.95$

-p nprocs

-v nvprocs

$-i$ interface

-C CM_name

progname

output_file

$\&$ causes $95 \%$ of the memory per virtual processor (vp) to be available (default is 0.75 )

attach nprocs CM processors; legal values for nprocs: $4 \mathrm{k}$ or $8 \mathrm{k}$ for BAMBI, (default is $4 \mathrm{k}$ ) $8 \mathrm{k}$ or $16 \mathrm{k}$ for GODZILLA (default $8 \mathrm{k}$ ) $8 \mathrm{k}, 16 \mathrm{k}$ or $32 \mathrm{k}$ for SON-OF-CUBE (default $8 \mathrm{k}$ )

configure the $\mathrm{CM}$ to have nvprocs virtual processors (must be at least the number of physical processors allocated)

attaches the CM via interface 0 (BAMBI) or interface 1 (GODZILLA) on NRL CM

attaches to the CM named 'CM_name' (use -C S for SON-OF-CUBE at NPAC)

your executable $\mathrm{C}^{*}$ program

file containing log of batch session

causes program to run in the background

The Connection Machine System C* User's Guide (section 4.3) includes instructions for executing $\mathrm{C}^{*}$ programs interactively.

\subsection{Re-configuring wave_3d}

The wave_3d program is currently configured for models with dimensions $64 \times 64 \times 64$, which is the largest model that will run on a $16 \mathrm{k}$ Connection Machine. The $32 \mathrm{k}$ Connection Machine at NPAC can accomodate a model with dimensions $64 \times 128 \times 64$. To change the model dimensions in the wave_3d program:

1. edit the header file wave_3d.hs and modify any or all of the following lines (note that model dimensions MUST be a power of 2!):

$\begin{array}{lll}\text { \#define } & \text { XDIM } & 64 \\ \text { \#define } & \text { YDIM } & 64 \\ \text { \#define } & \text { ZDIM } & 64\end{array}$

2. re-compile the wave_3d program (refer to section 4.6)

cmx\% cs - O -w -o wave_3d wave_3d.cs nd_grid.o fb.o \&

3. edit xmodel.dat to incorporate the new model parameters 
4. attach and run the program, making the appropriate changes to the cmattach parameters (refer to section 4.7)

cmx\% cmattach -b 0.95 -p $32 \mathrm{k}-\mathrm{v}$ 512k -C S wave_3d > wave_3d.out \&

\section{Frame Buffer}

The Connection Machine graphic display system consists of a framebuffer and a high resolution color monitor. The framebuffer contains a display memory capable of storing an image 2048 by 1024 pixels with three eight-bit color buffers and a four-bit overlay buffer. The color monitor has a resolution of 1280 by 1024 pixels. Since the display memory can store more pixels than the monitor can display, pan and zoom operations are supported.

The CM graphic display system allows each virtual processor to be mapped to a pixel on the color monitor. As values from the virtual processors are written to the display memory, the image on the monitor changes. For the 3D synthetic seismograms, a 2-dimensional plane is selected (for example, the $\mathrm{xz}$ plane) and 'slices' through the model are displayed at selected time steps in 'real-time'.

The framebuffer is normally accessed via C/PARIS (Connection Machine PARallel Instruction Set) calls. The wave_3d program uses the NRL $\mathrm{C}^{*}$ library $\mathrm{fb}$ which is a collection of functions used to display pixels on the framebuffer (see Appendix A). To use these functions in a $\mathrm{C}^{*}$ program, include the header file:

\#include "fb.cs"

and link with the library: -lnrlcstar (if using NRL Connection Machine) or $1 b . \circ$ (if using NPAC Connection Machine).

\section{DataVault}

The DataVault is a 5-Gigabyte mass storage system which provides a data parallel file system for the Connection Machine. Data can be transferred to and from the CM-2 in parallel at high speed (40 megabytes per second) or it can be read and written directly from the front-end without using the CM-2.

The amount of memory per processor and the number of physical processors on the Connection Machine constrains the maximum size of the $3 \mathrm{D}$ model that can be computed.

In order to calculate $3 \mathrm{D}$ synthetic seismograms for models with dimensions of $128 \times 128 \times 128$ (actual dimensions $124 \times 128 \times 124$ with overlap), the model can be divided into four segments with an overlap of 4 grid points (figure 11). Each segment can be copied to a temporary file on the DataVault at each time step. Preliminary calculations indicate that approximately 2 seconds per time step are required to write the 23 parallel domain variables to four temporary files on the DataVault. 


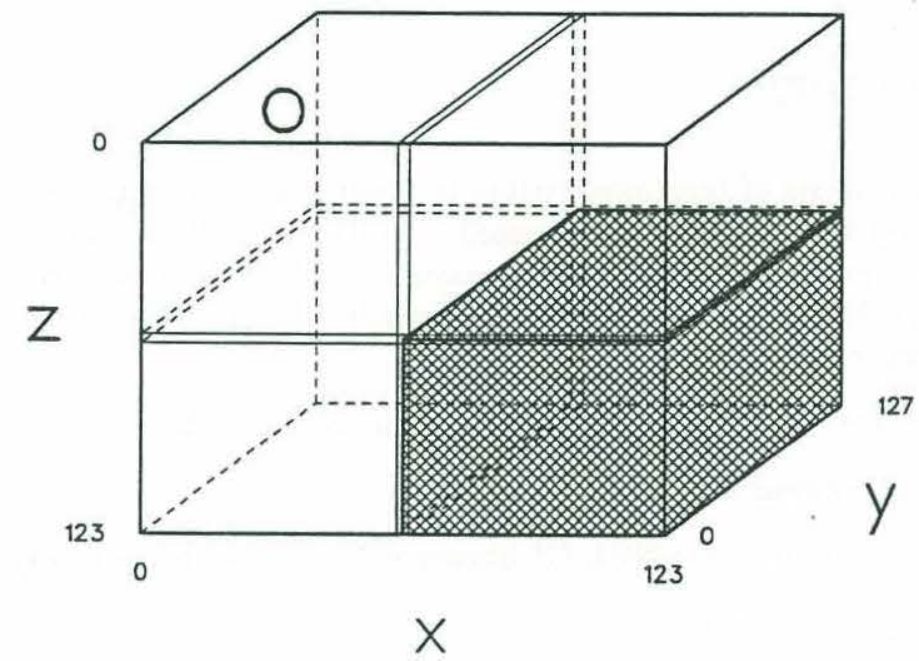

Three-dimensional model divided into four segments; each segment has dimensions $64 \times 128 \times 64$. The segments overlap four grid points in the $\mathrm{x}$ - and $\mathrm{z}$ directions, giving total model dimensions of $124 \times 128 \times 124$. At every time step, the variables calculated for each segment will be stored on the DataVault.

Figure 11: Segmentation of large model using DataVault 


\section{Appendices}

\section{A NRL cstar library}

The NRL cstar library consists of functions written to provide a simple interface to many of the Connection Machine features that can only be accessed directly via $\mathrm{C} / \mathrm{Paris}$. The library modules were written to solve specific problems encountered by users at NRL. A full description of the library can be found in the README file located in the nrl-cstar-lib directory on the NRL and NPAC front-end computers (cmvax, cmsun and $\mathrm{cmx}$ ).

The NRL C* library resides on the NRL front-end computers in the directory:

/cm-lights/library/nrl-cstar-lib

On the NPAC front-end computer, the NRL C* library is located in the directory:

/public/cm-lights/nrl-cstar-lib

\section{A.1 N-dimensional grid}

The finite differences method uses many 'nearest-neighbor' calculations. Although $\mathrm{C}^{*}$ supports a NEWS package to perform nearest neighbor calculations in two dimensions, this package currently does not support three-dimensional NEWS communications. The nd_grid module of the NRL C* library provides fast nearest neighbor communications on $\mathrm{N}$-dimensional grids (up to 8 dimensions).

For more information on the NRL C* n-dimensional grid library(figure 12):

cmx\% cd /public/cm-lights/nrl-cstar-lib/nd_grid

cmx\% more README

\section{A.2 Framebuffer}

The NRL C* library module fb is a simple interface to the high speed, high resolution CM graphics display system. This module consists of functions to initialize the framebuffer, set up a color table, and display pixels on the color monitor.

For more information on the NRL C* framebuffer library (figure 13):

cmx\% cd /public/cm-lights/nrl-cstar-lib/fb

cmx \% more README

\section{B cmusers group}

NRL supports a users' forum for exchanging questions, ideas and experiences among programmers using the Connection Machine Facility. Mail can be directed to cmusers and questions usually 

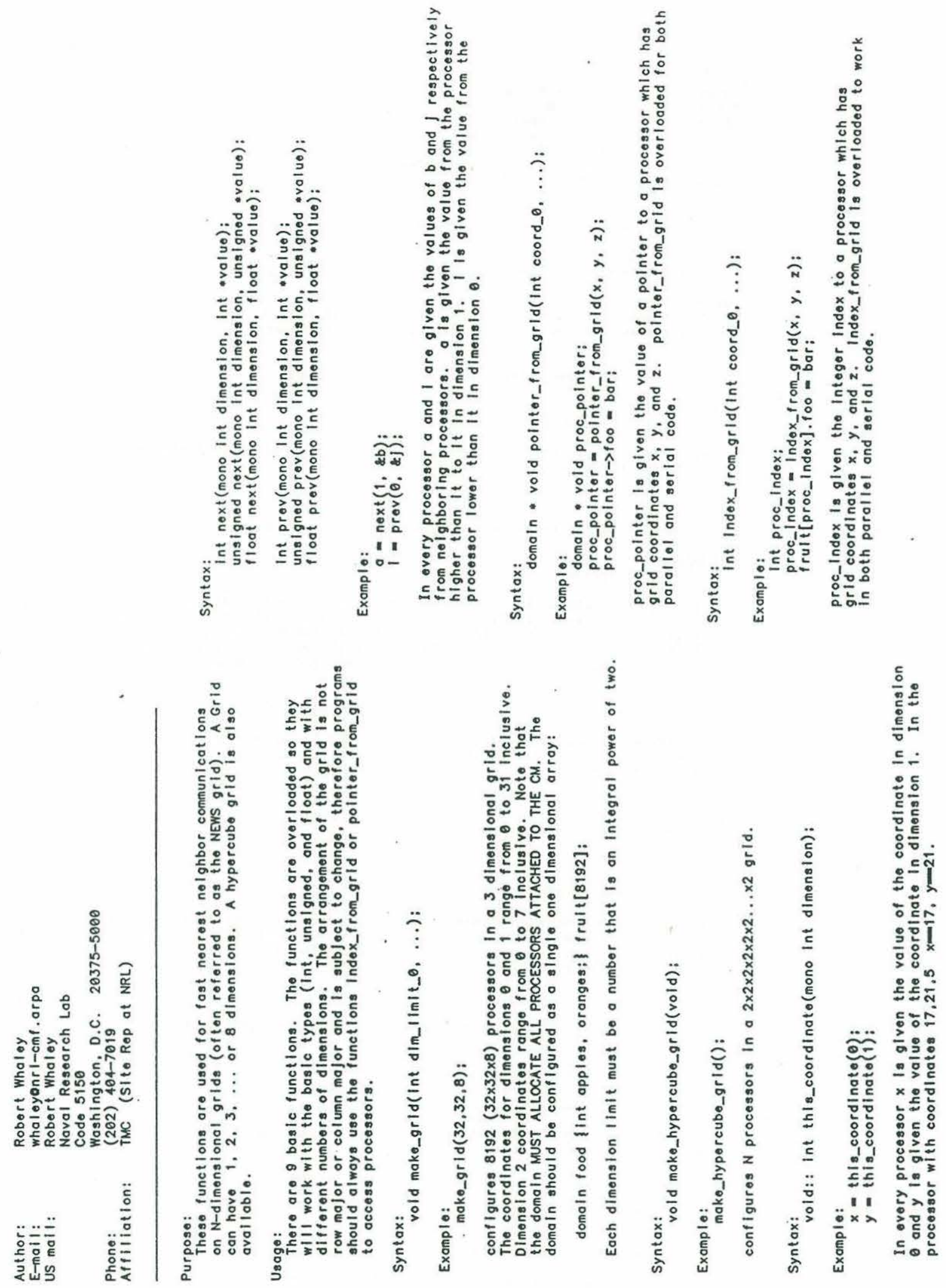


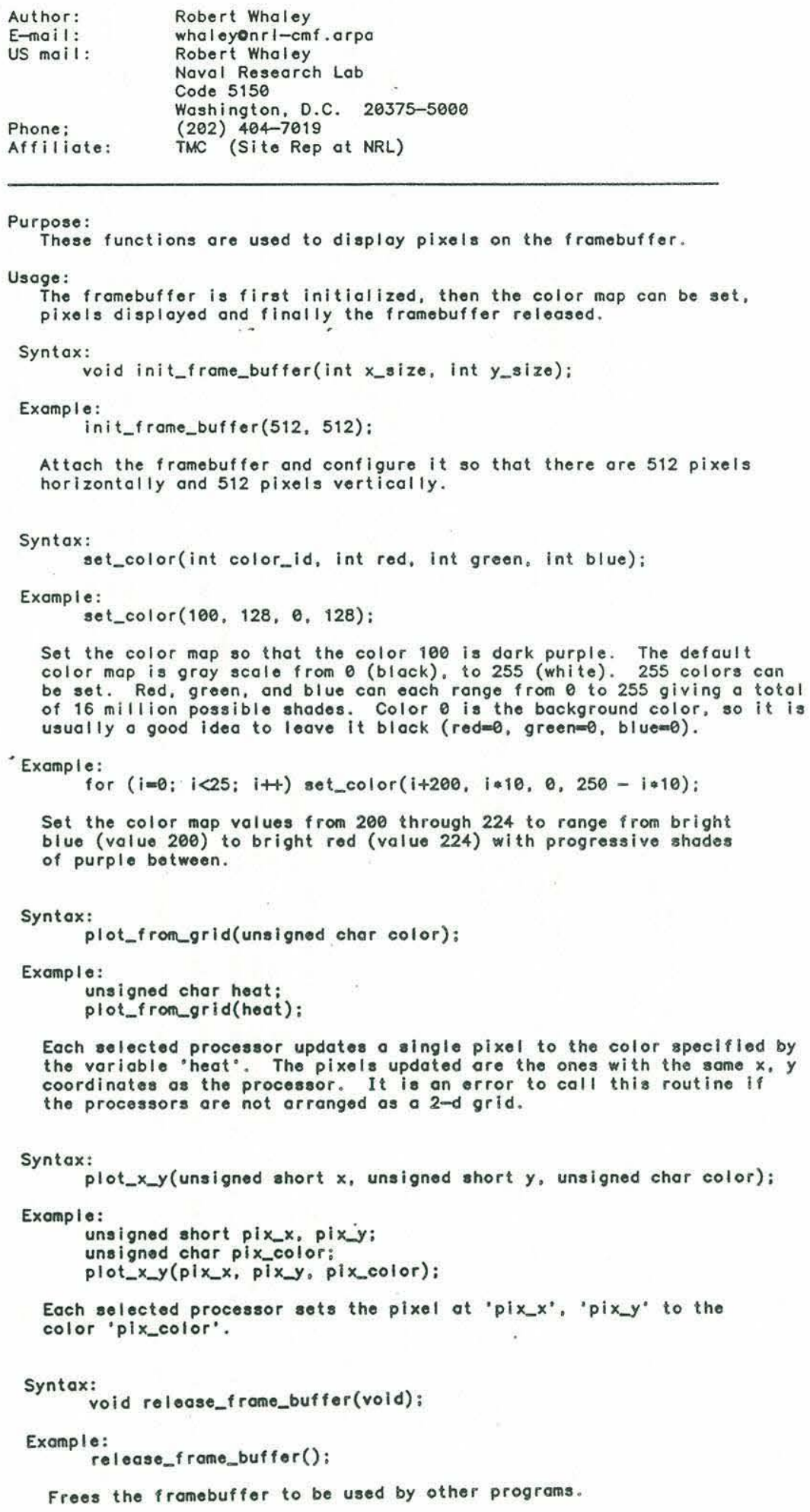

Frees the framebuffer to be used by other programs.

Figure 13: README file for fb library 
receive quick and thorough responses. Since all members of the cmusers group receive the mail, you can learn about problems/bugs with new compilers, upcoming classes and talks, innovative algorithms, etc. To join this forum, simply mail your request to be added to the mailing list to cmusers.

To get help on the mail facility:

\section{cmsun\% man mail}

NPAC supports an online consultant service. To get help, send mail to consult. 


\section{Structure diagram for wave_3d.cs}

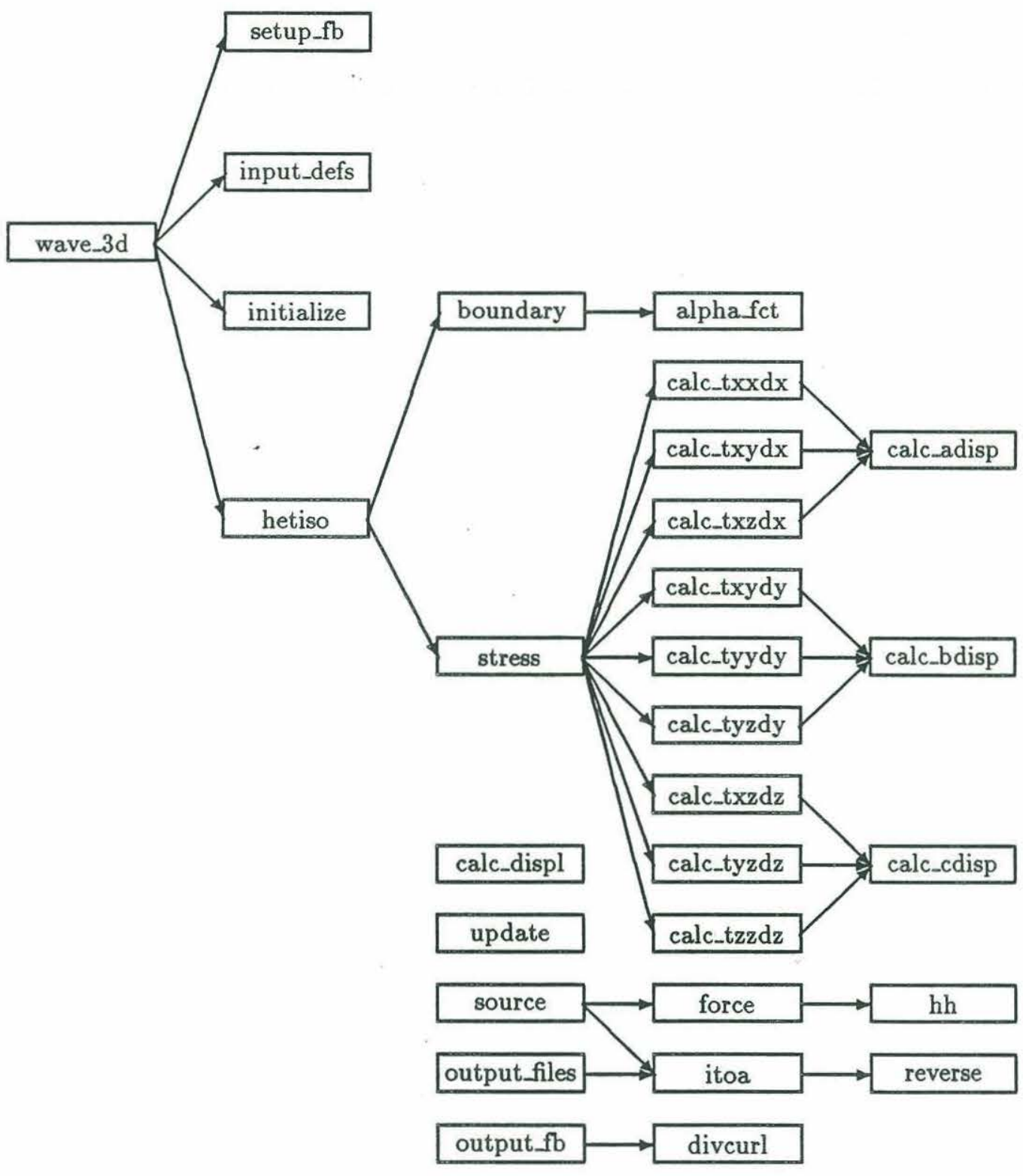




\section{Source Code}

\section{D.1 wave_3d program}

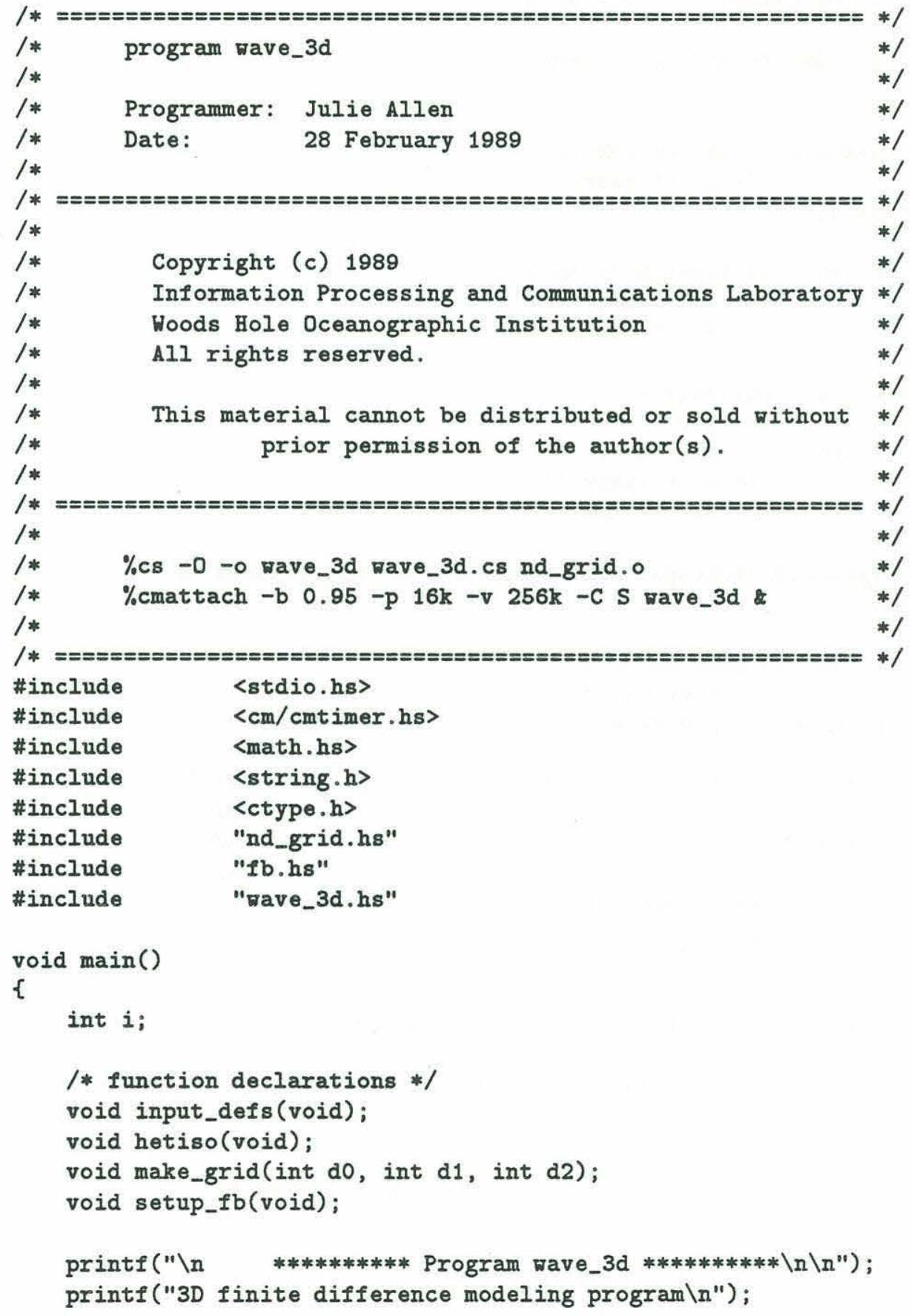




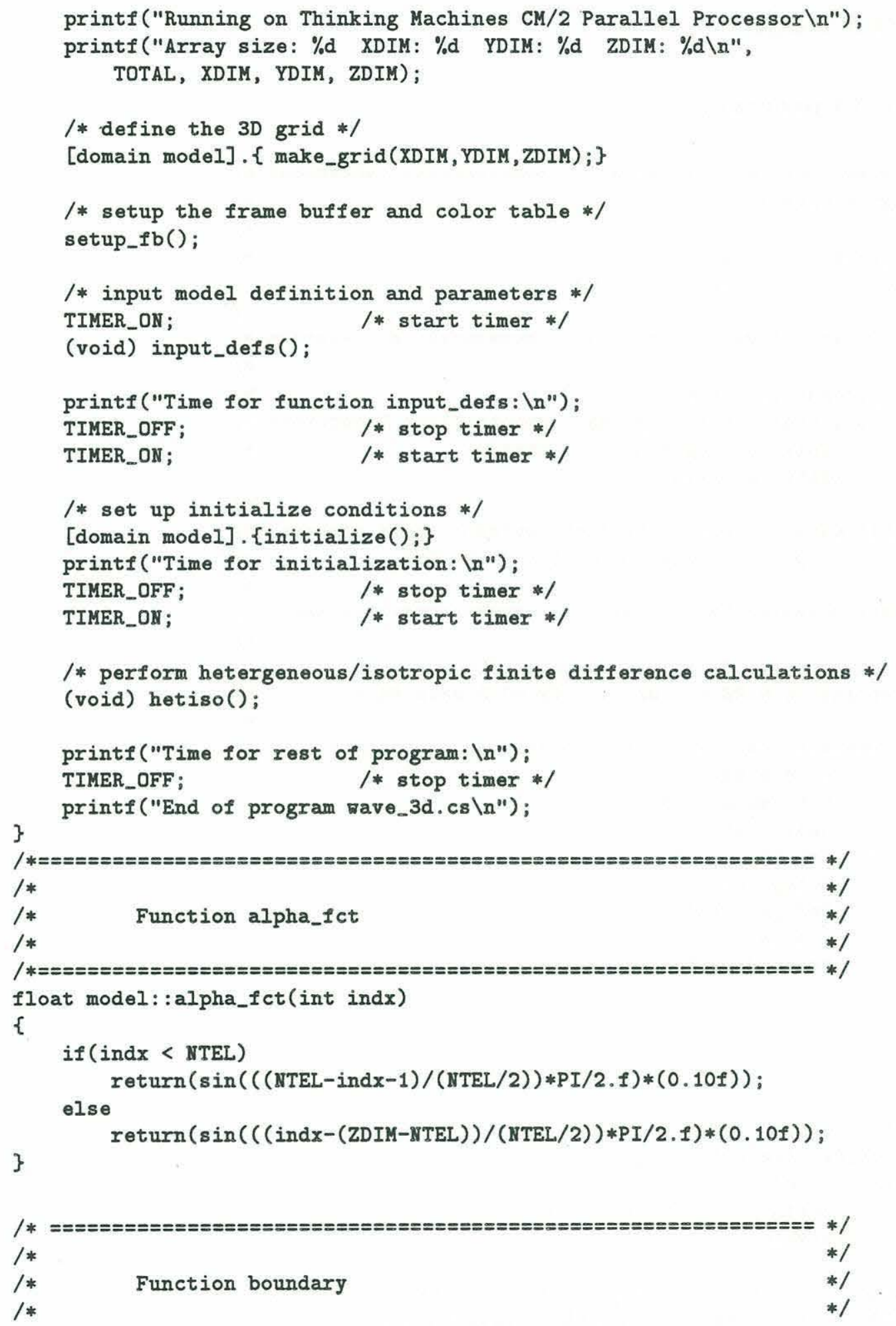




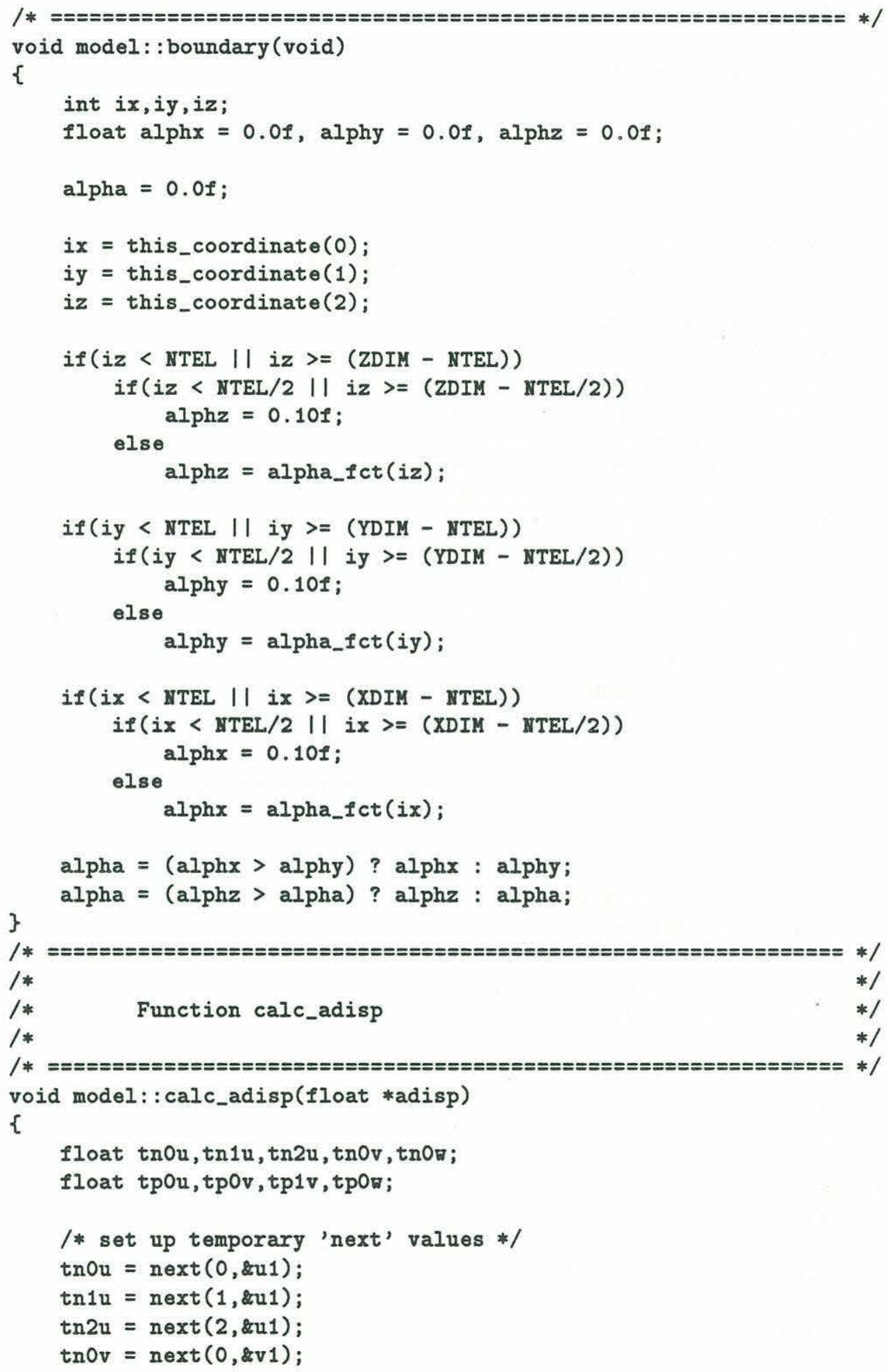




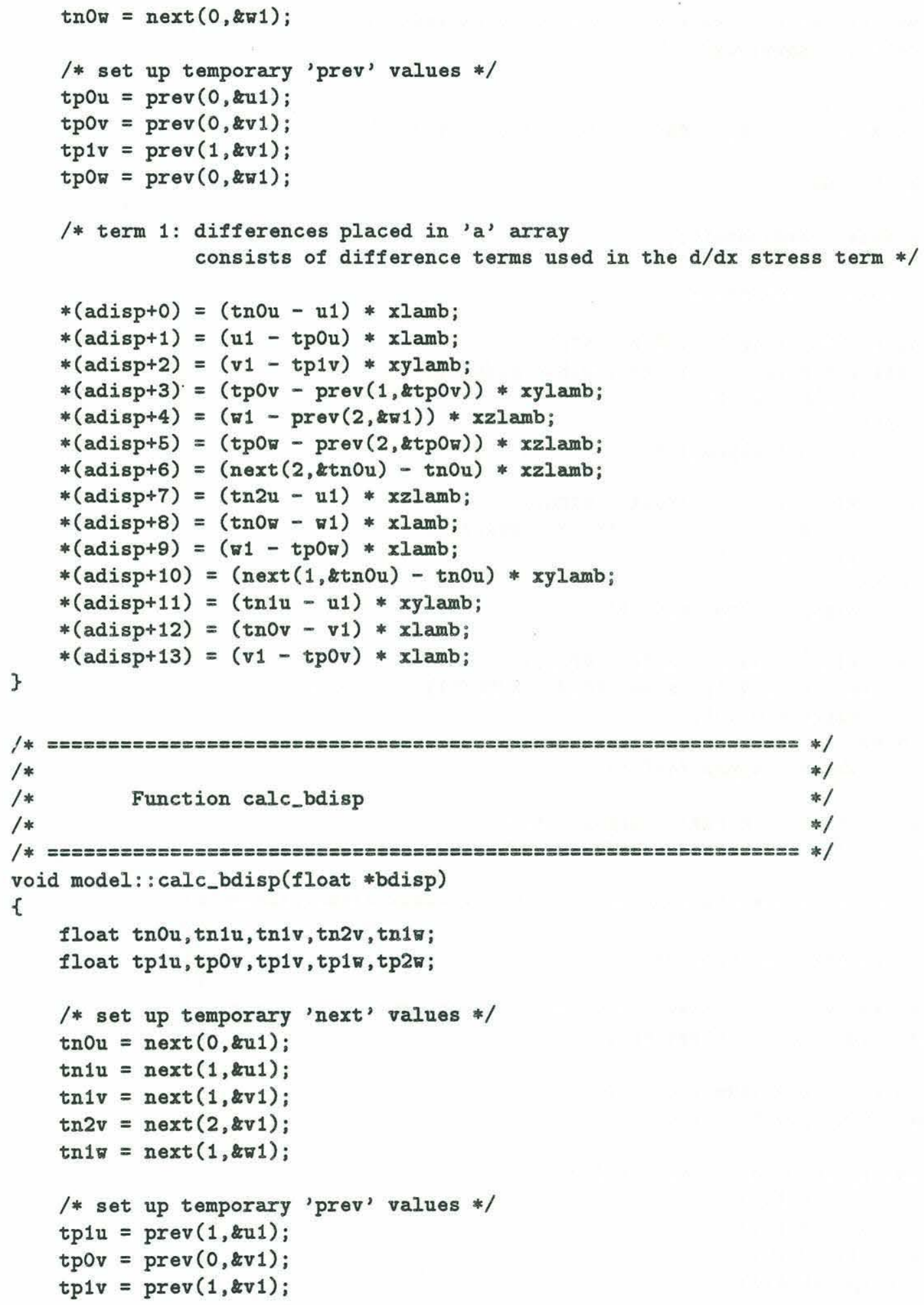




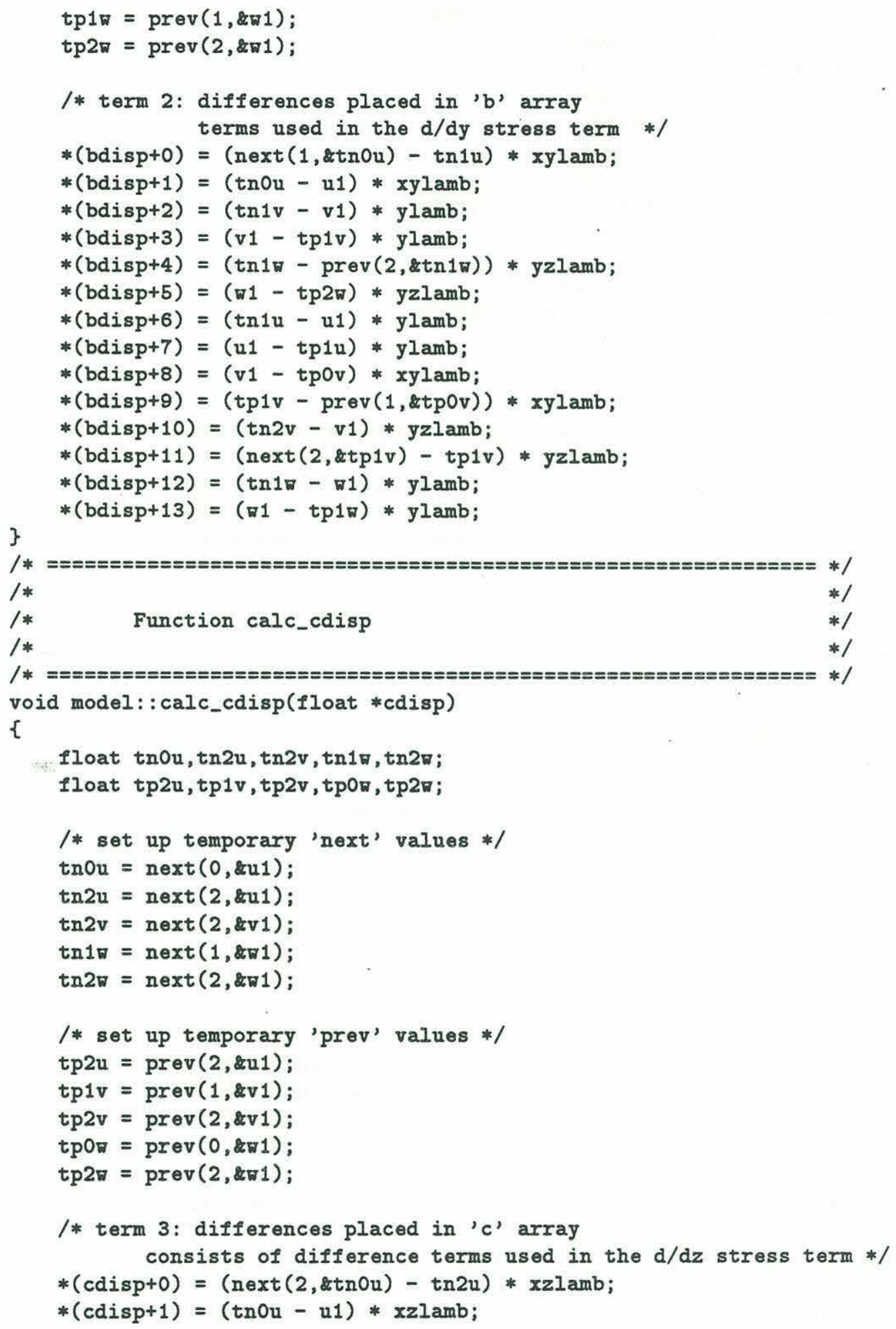




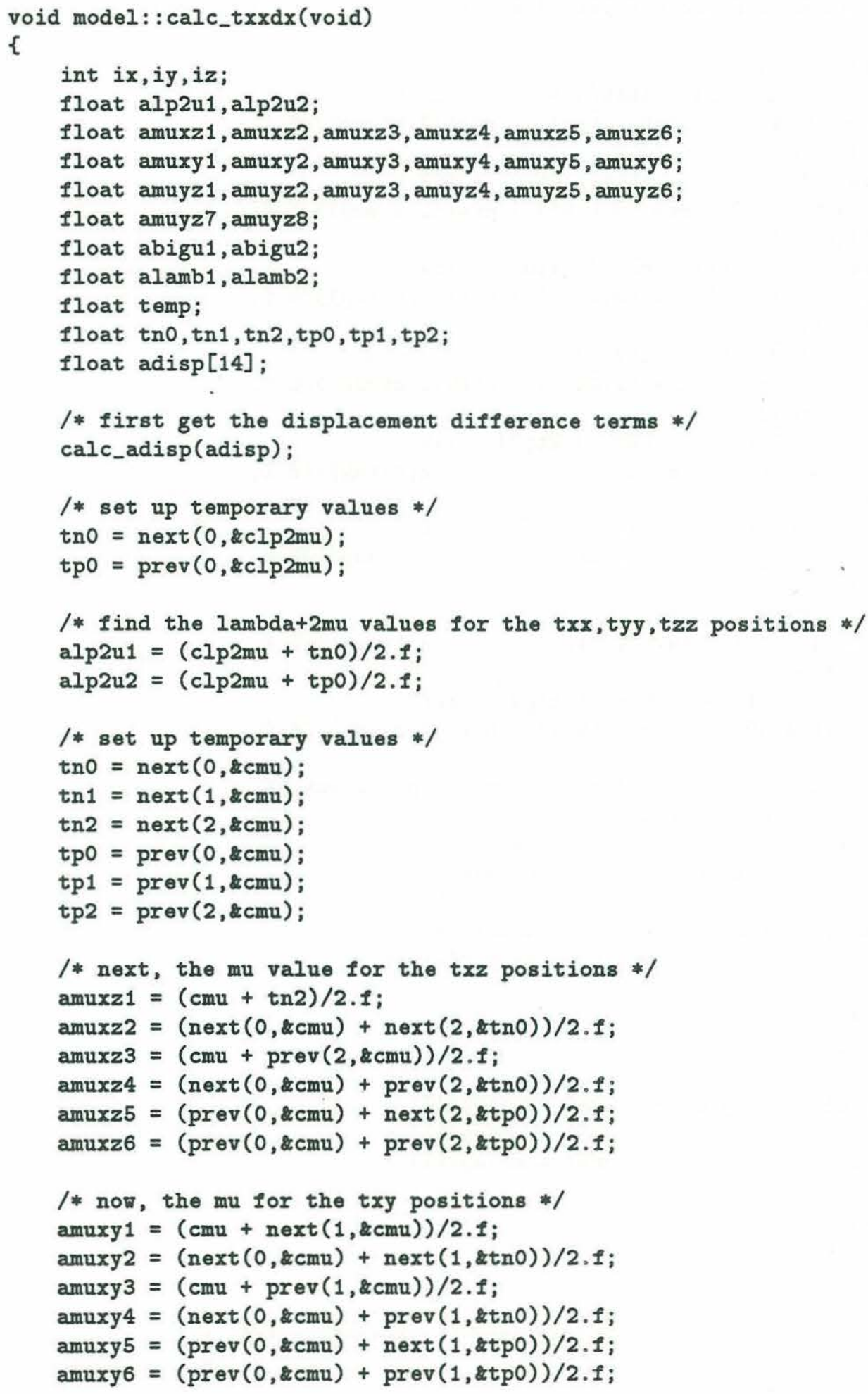




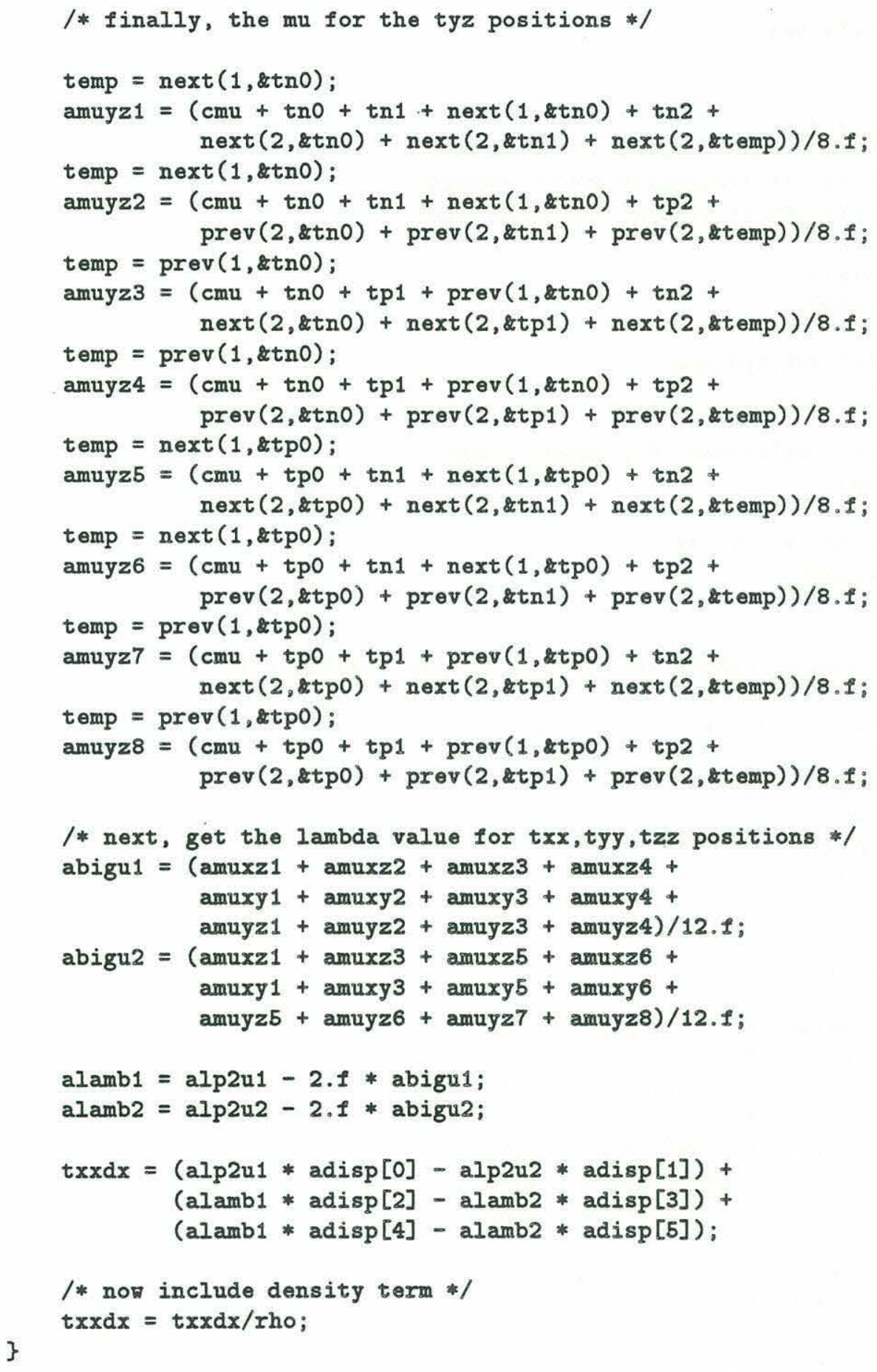




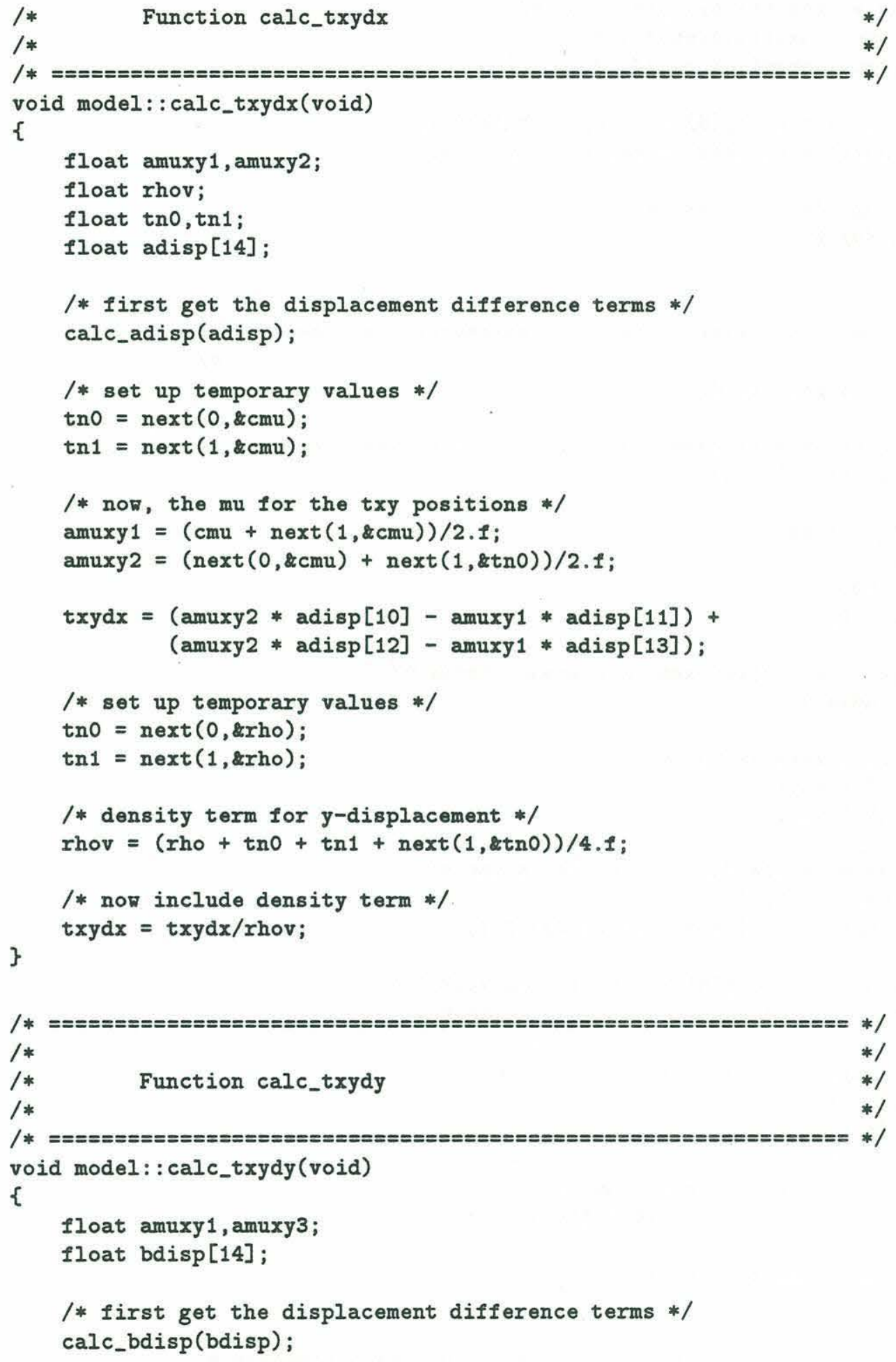




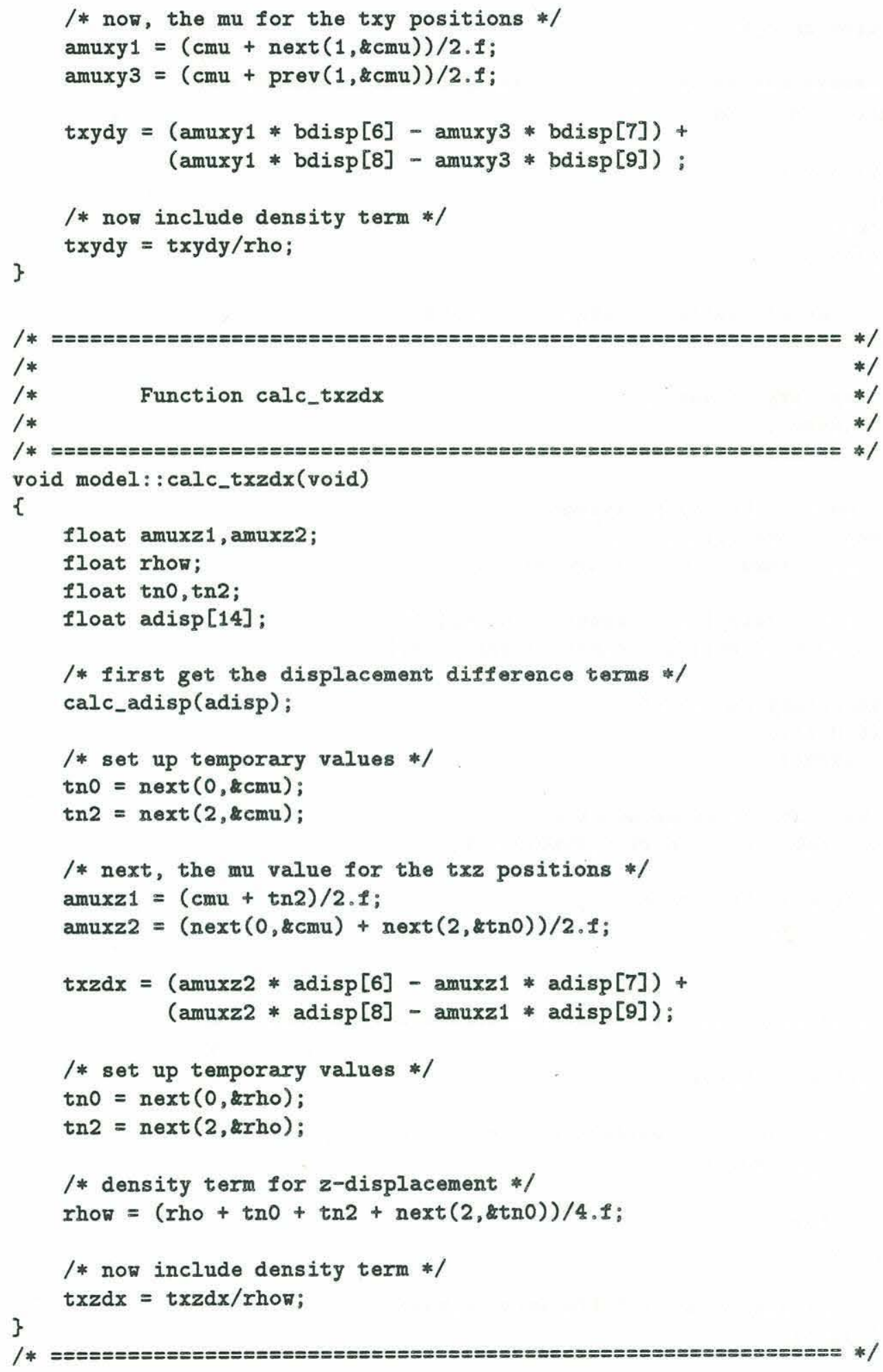




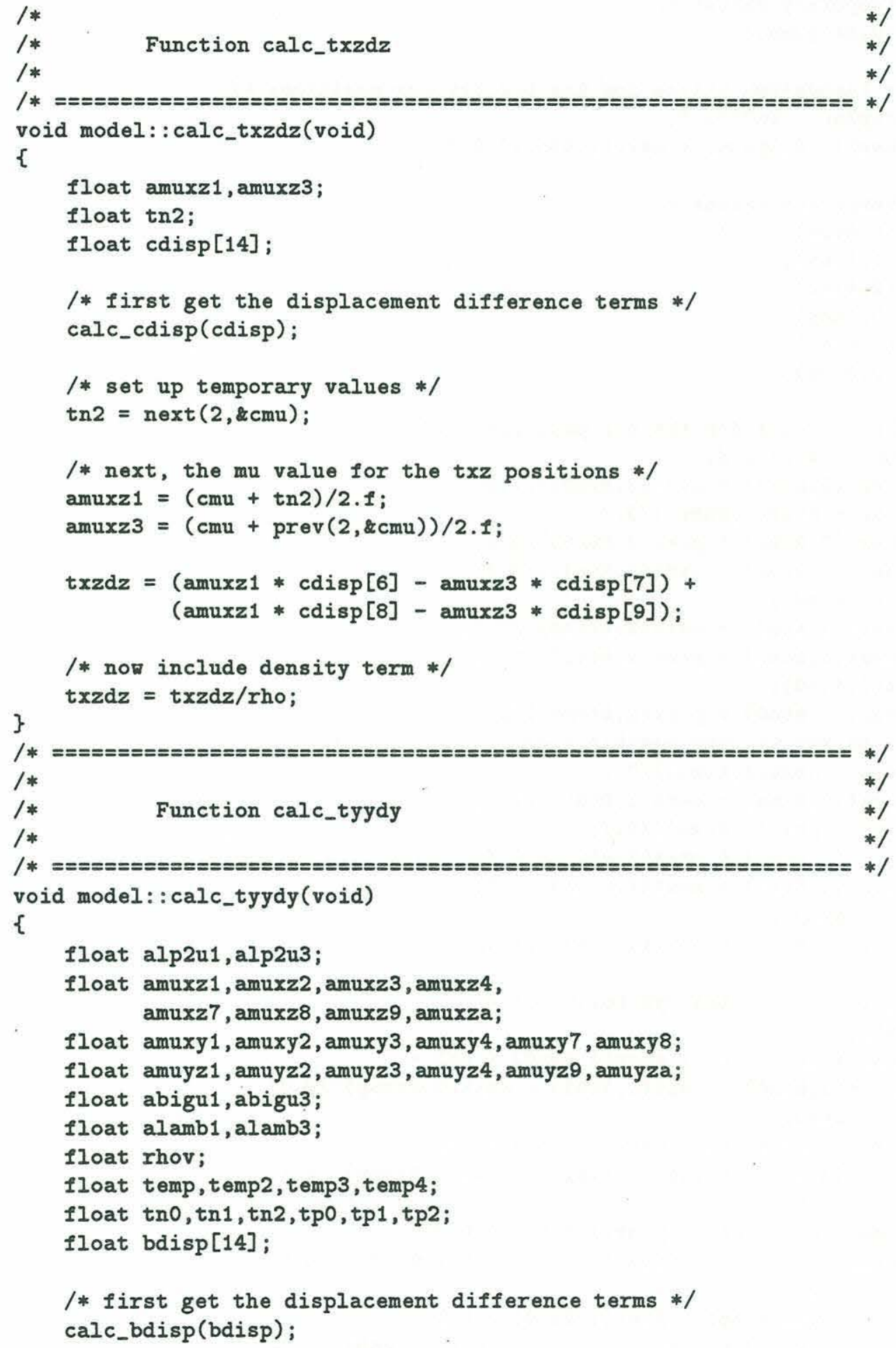




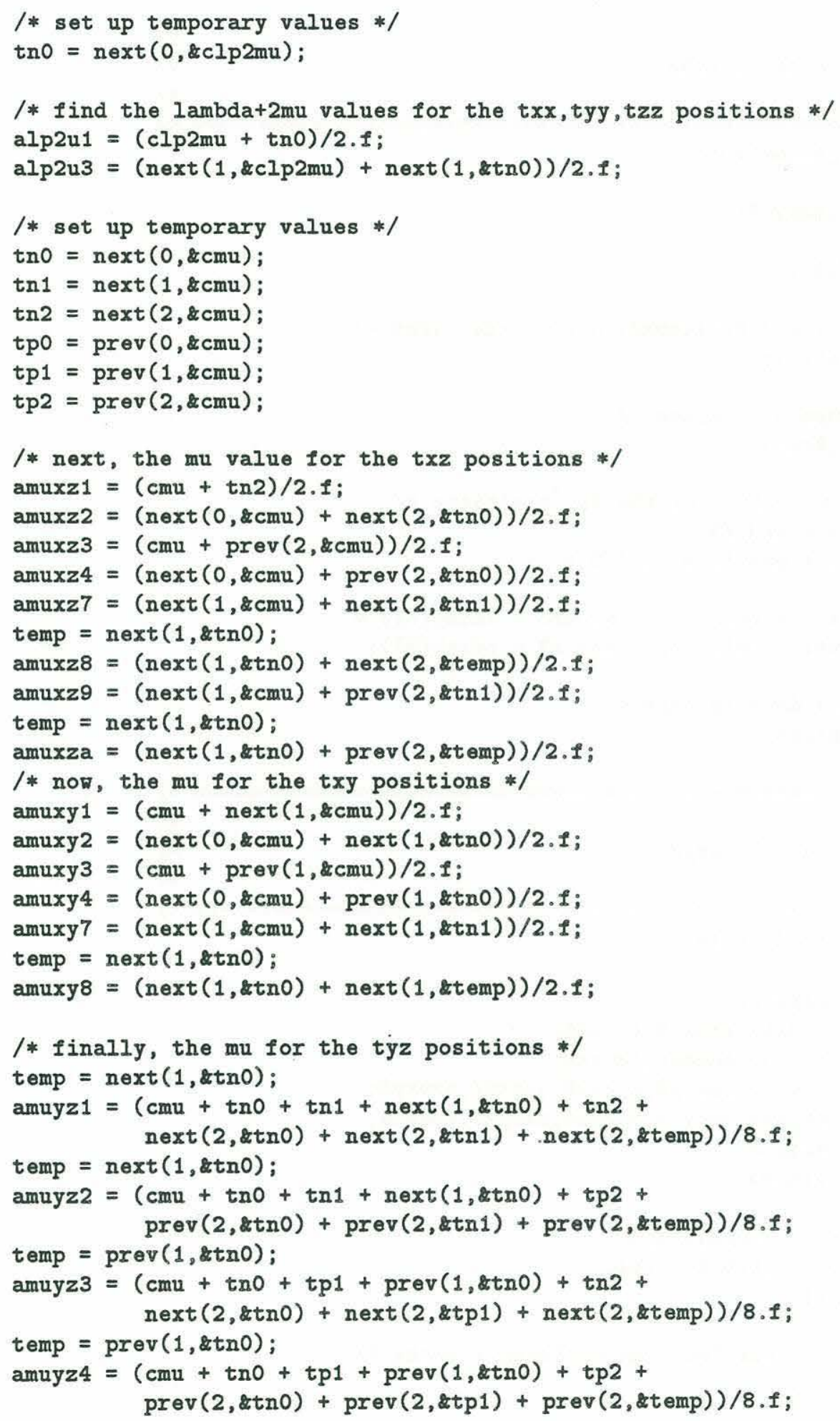




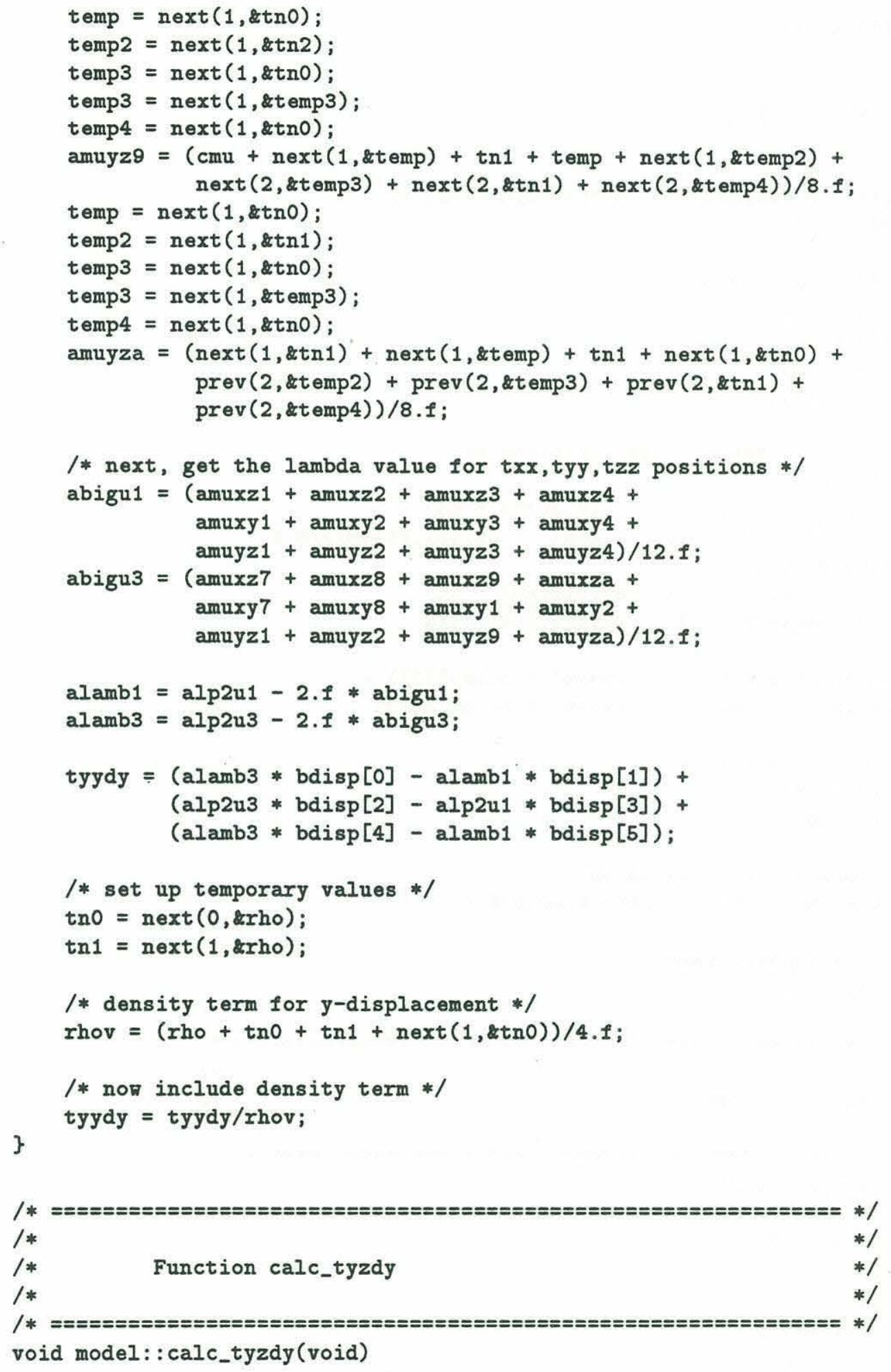




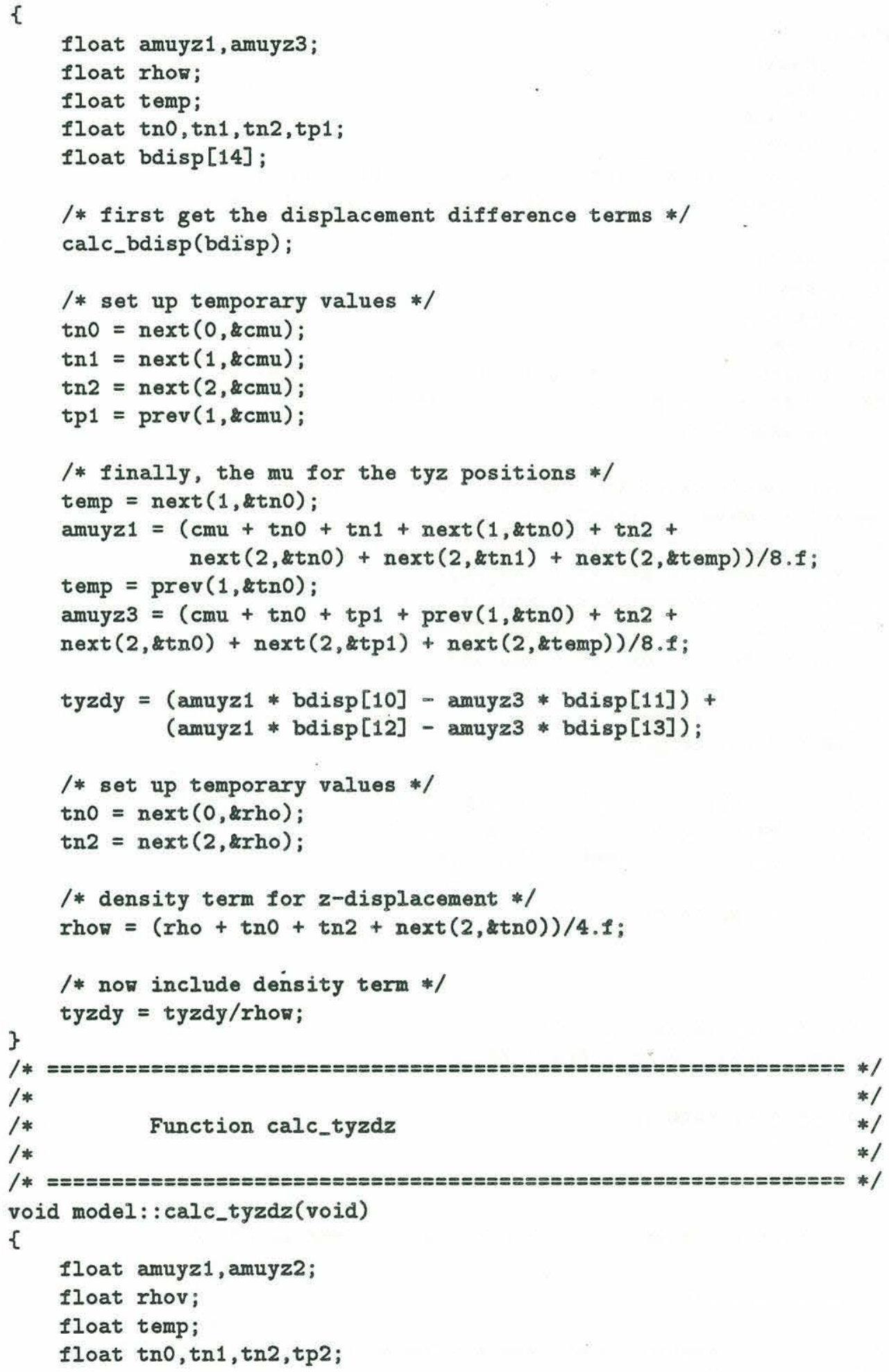




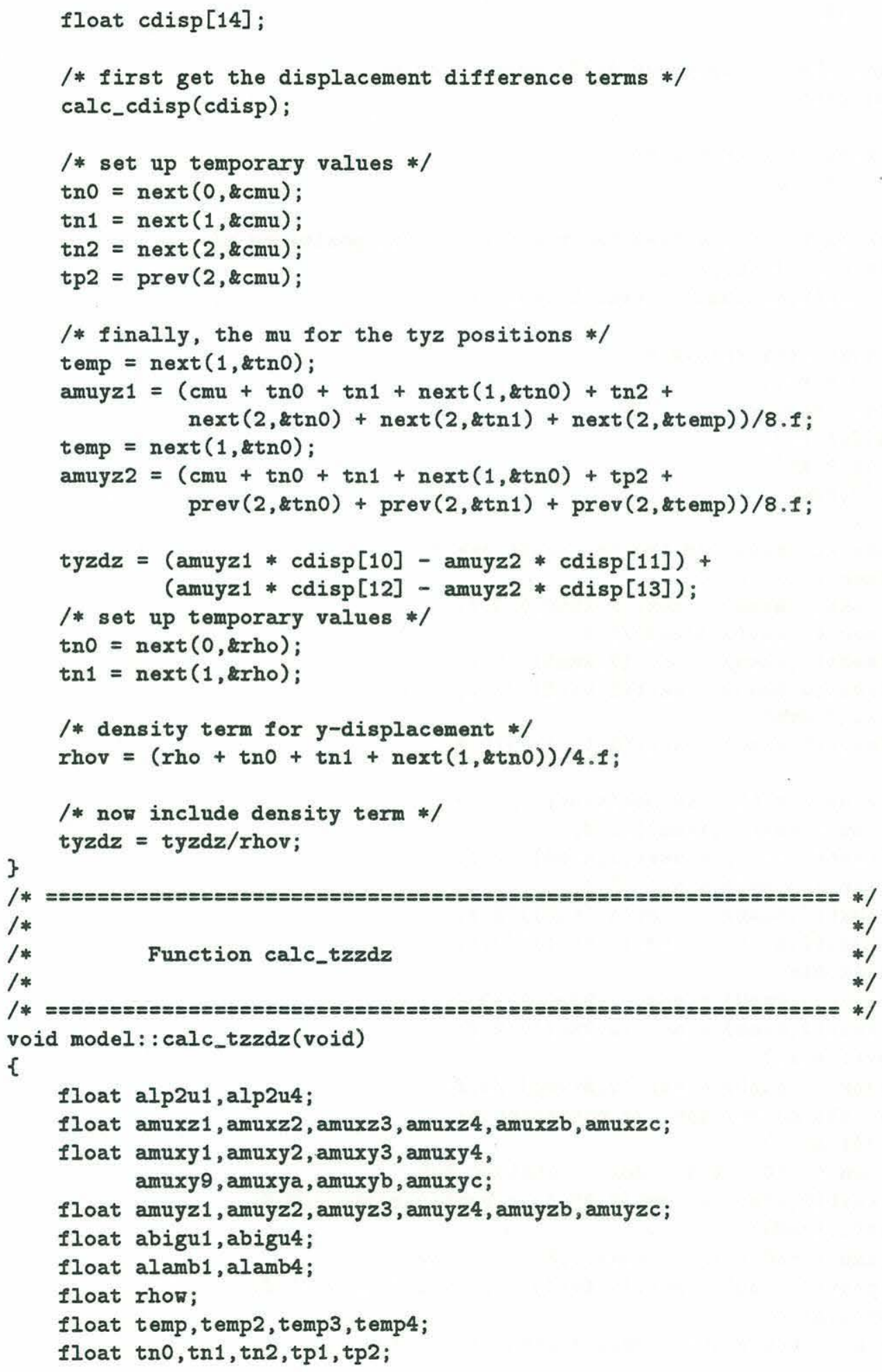




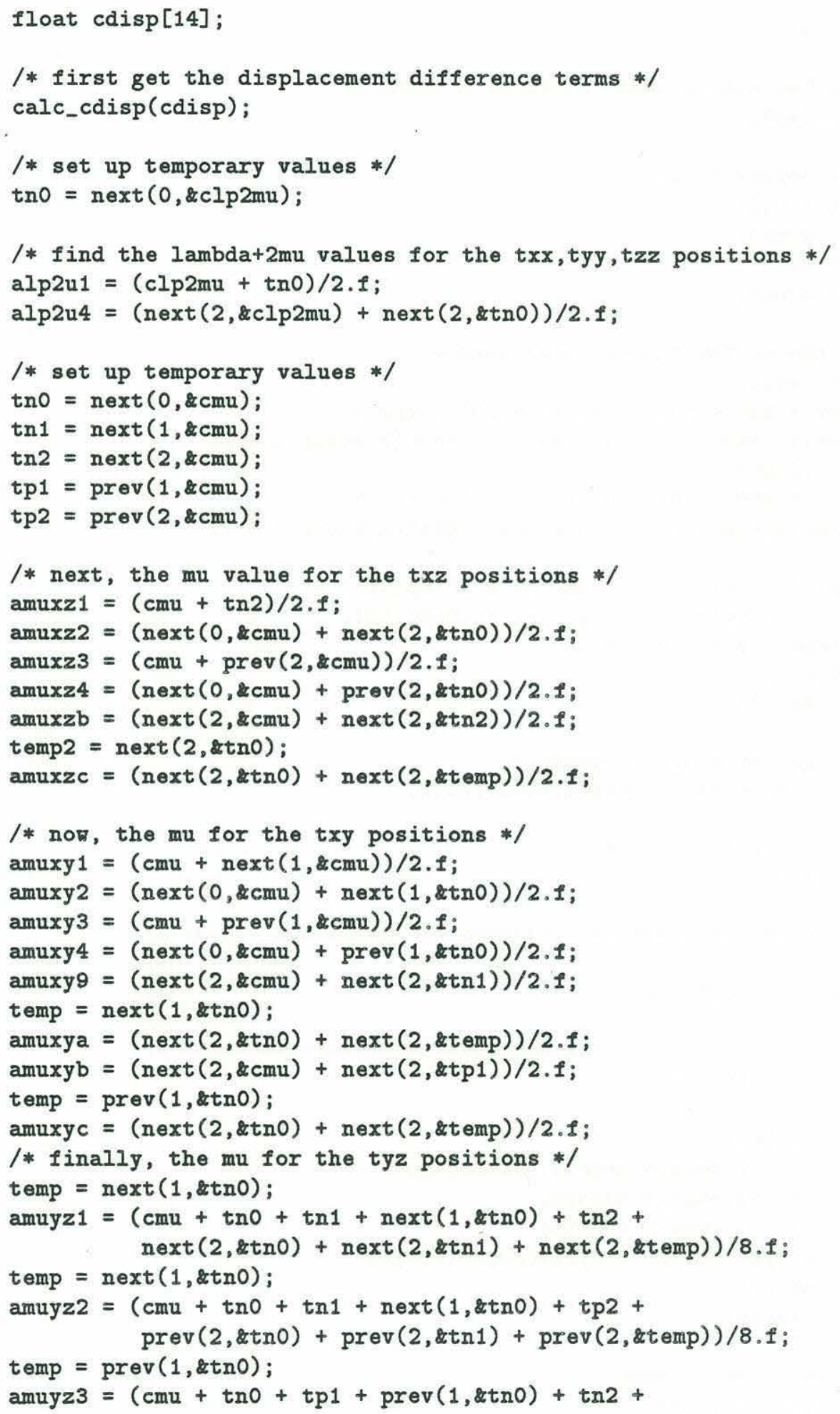




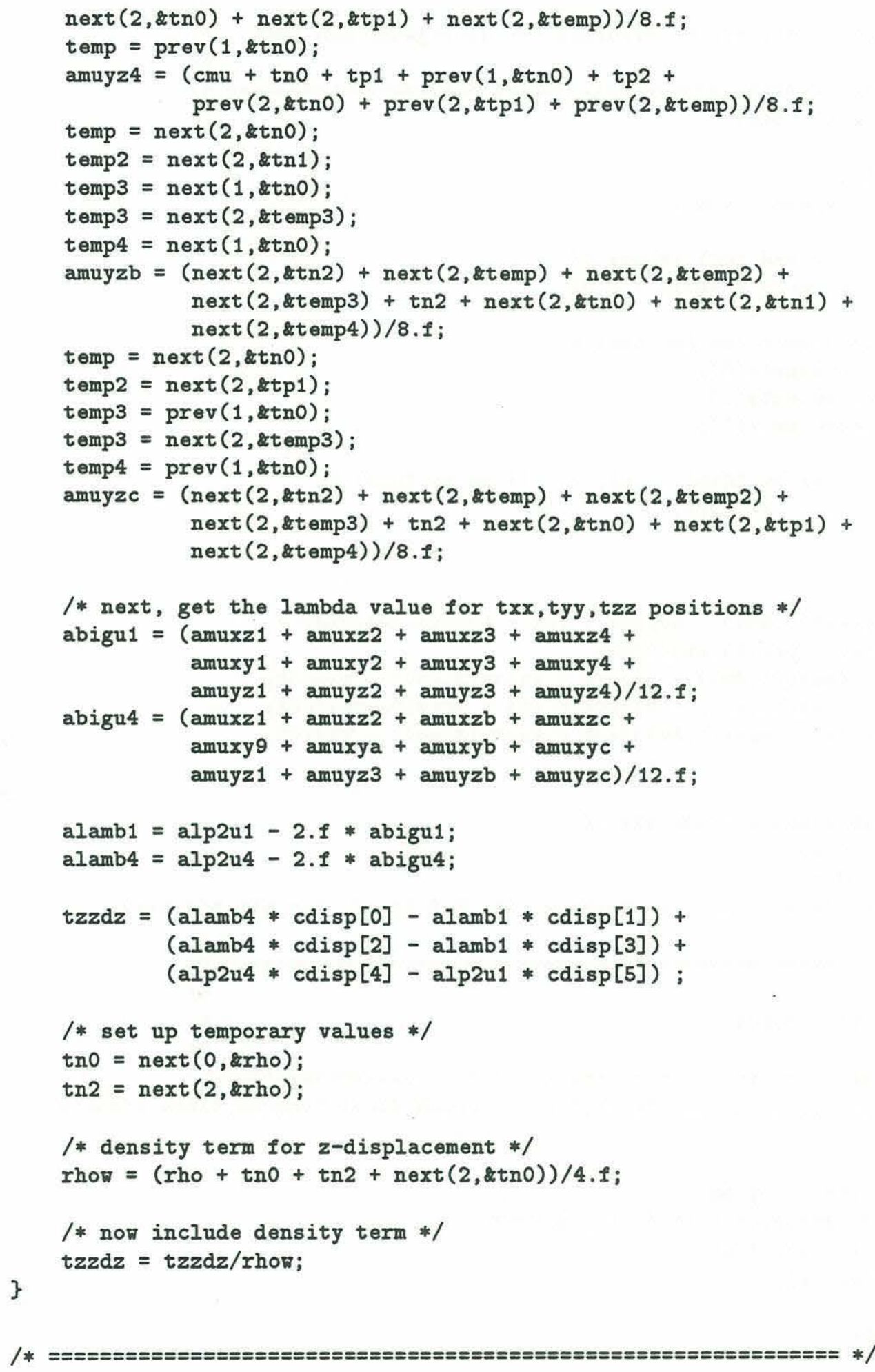




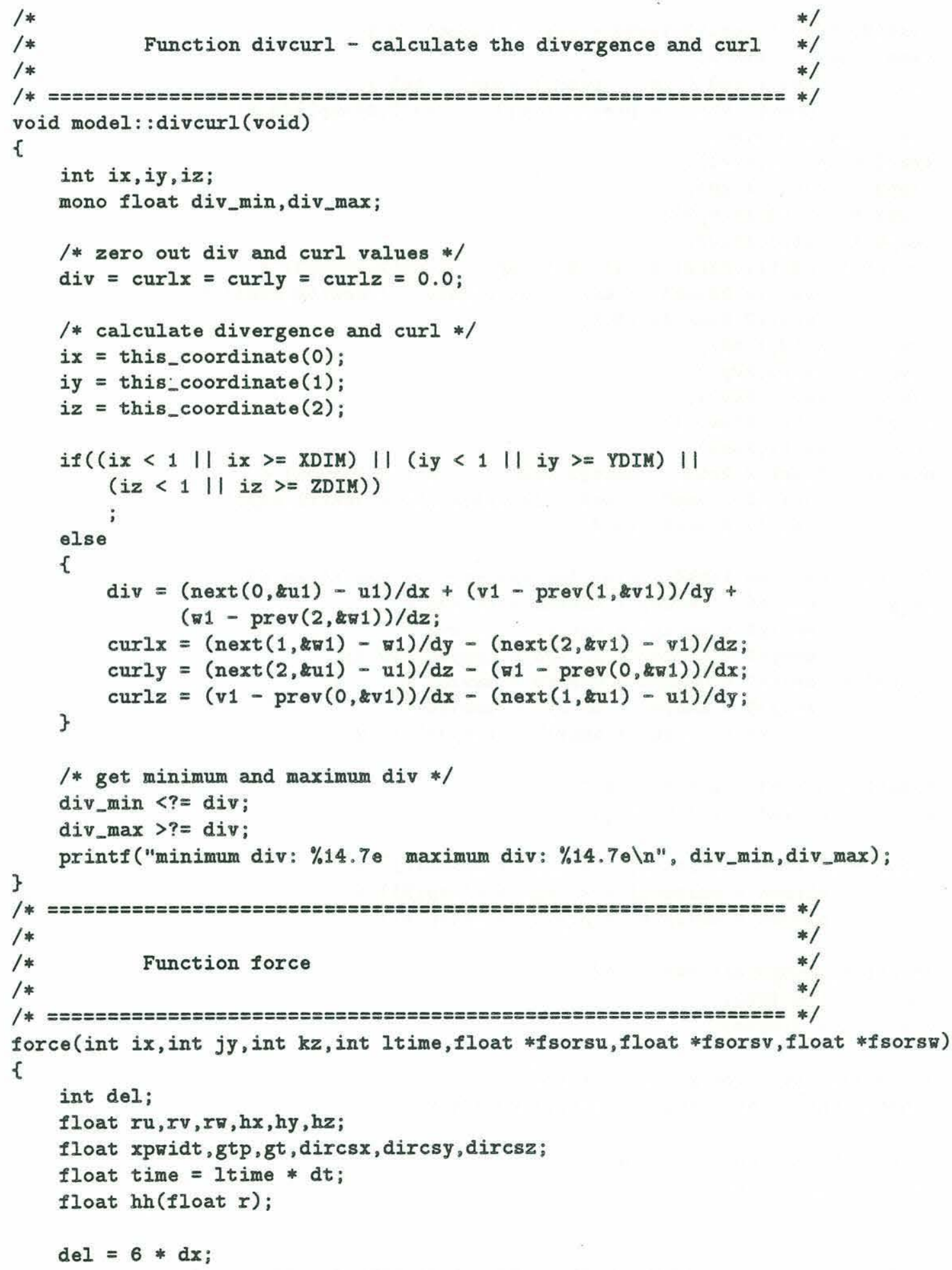




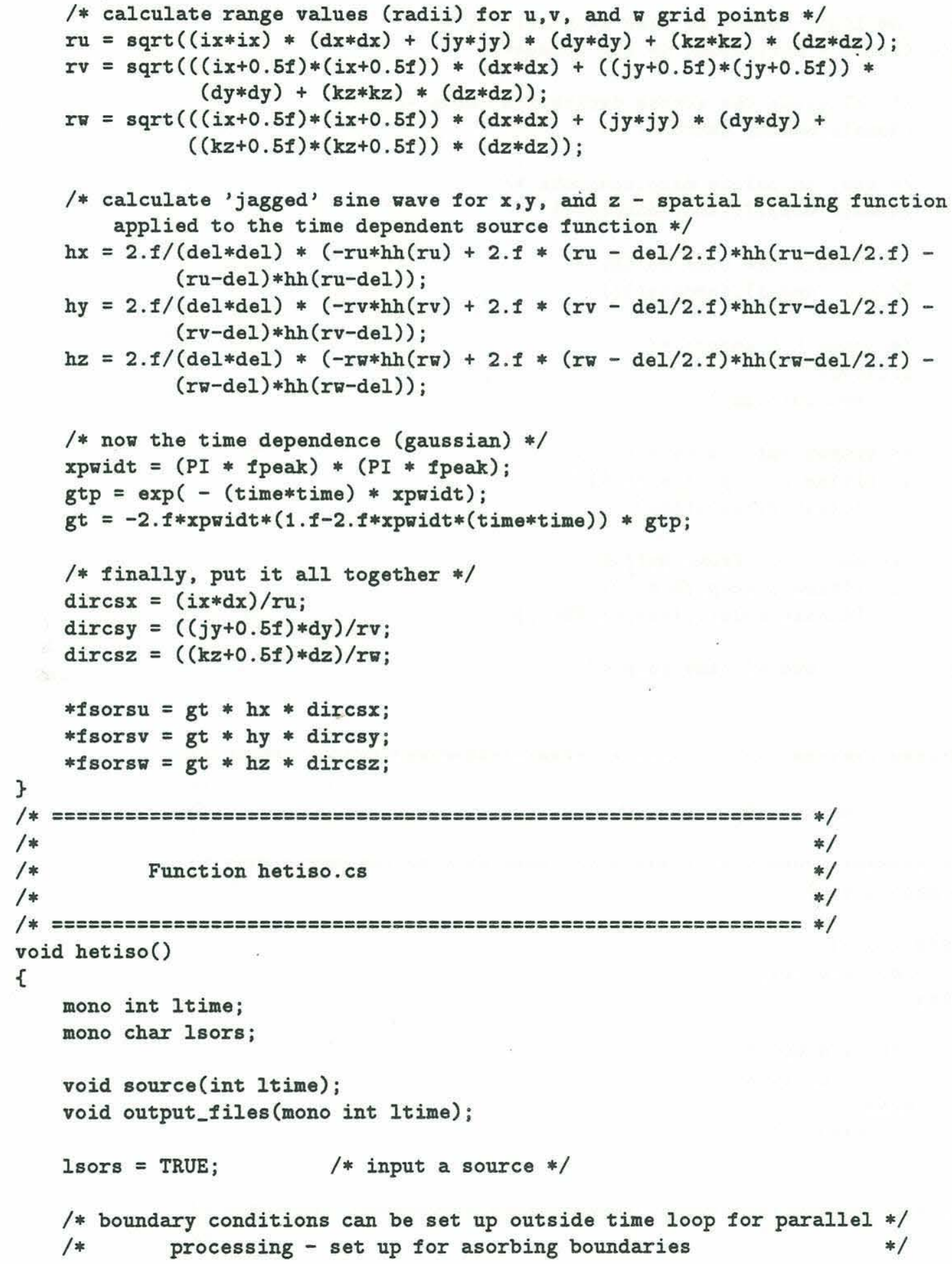




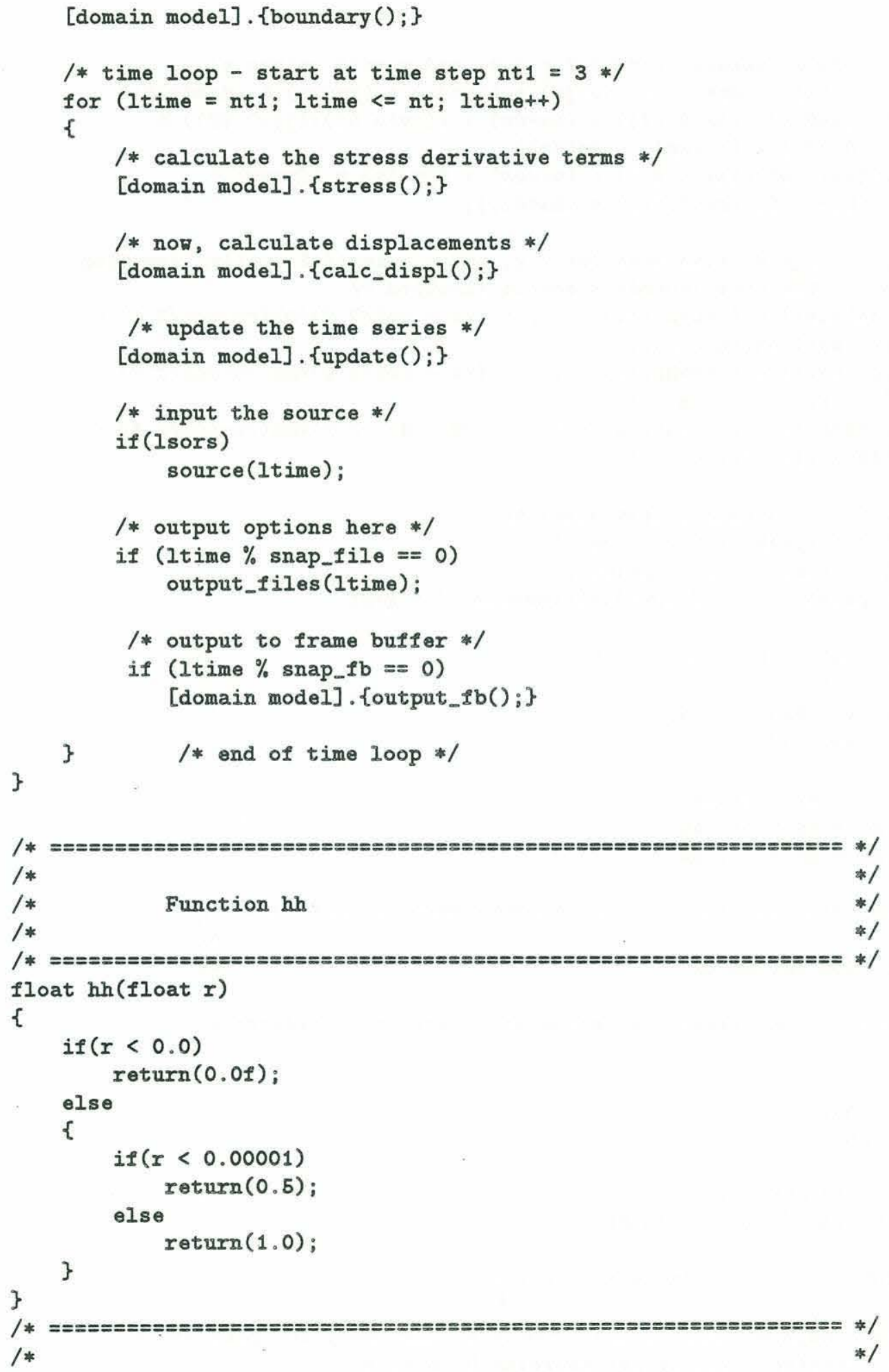




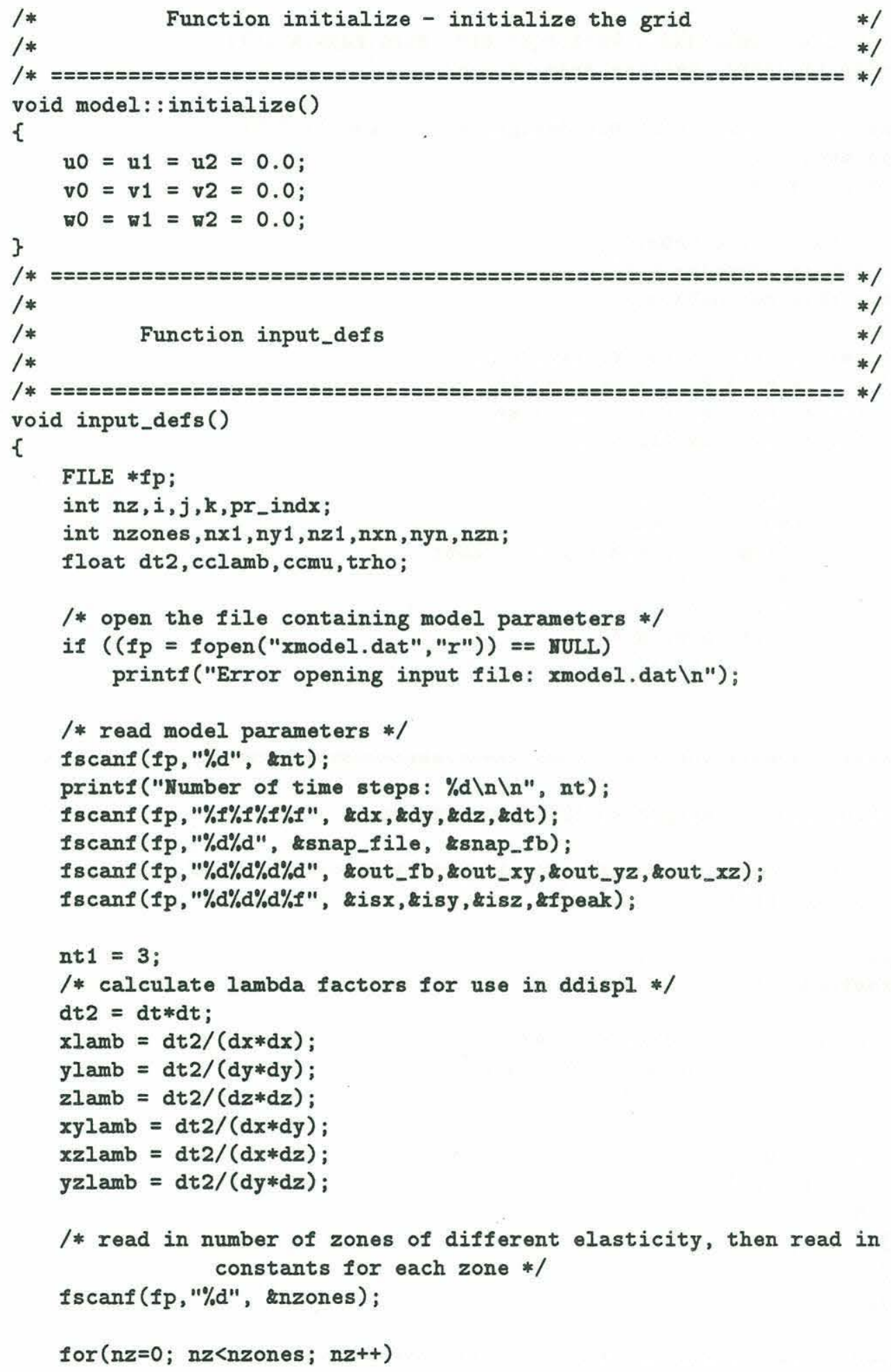




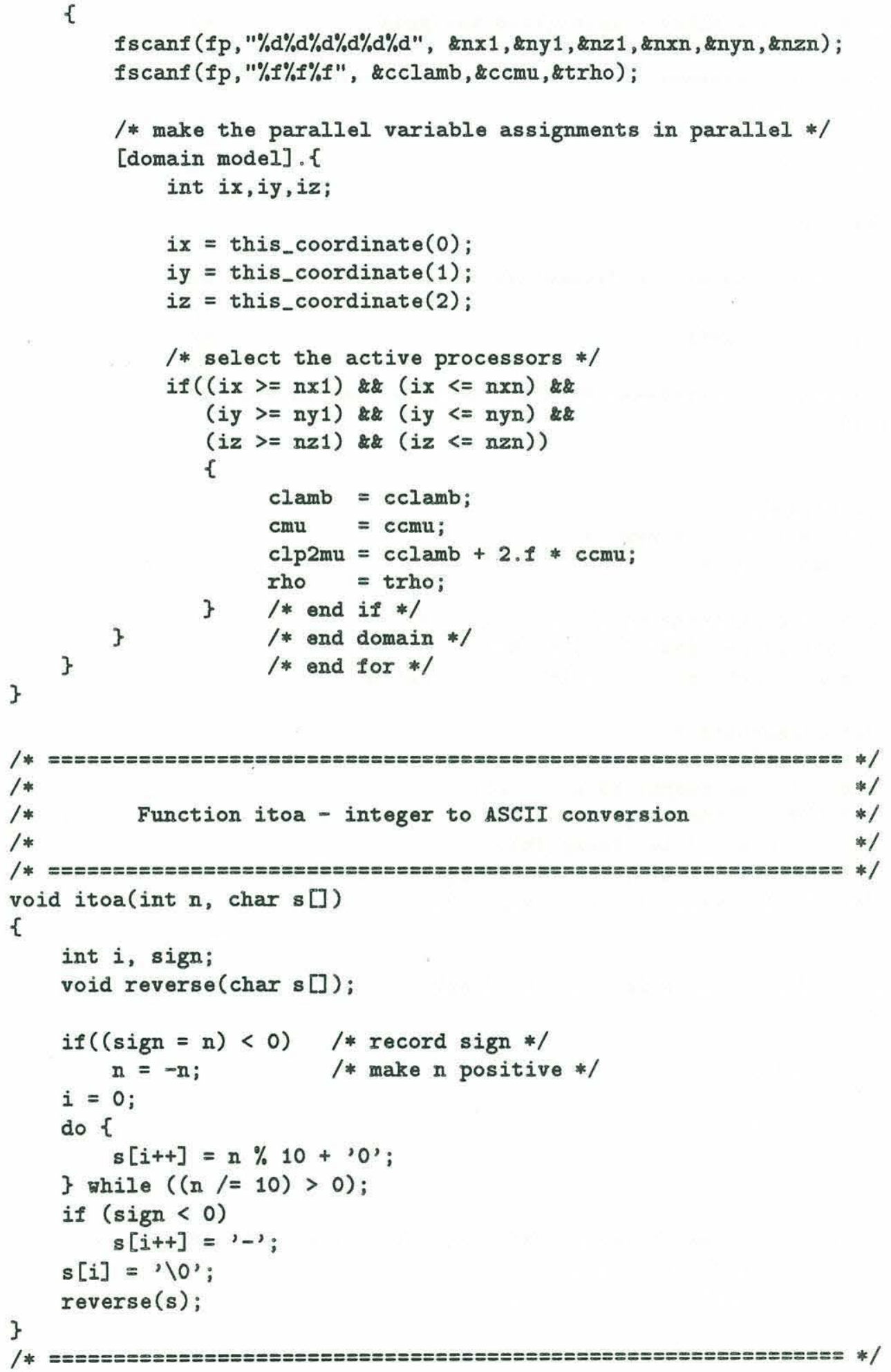




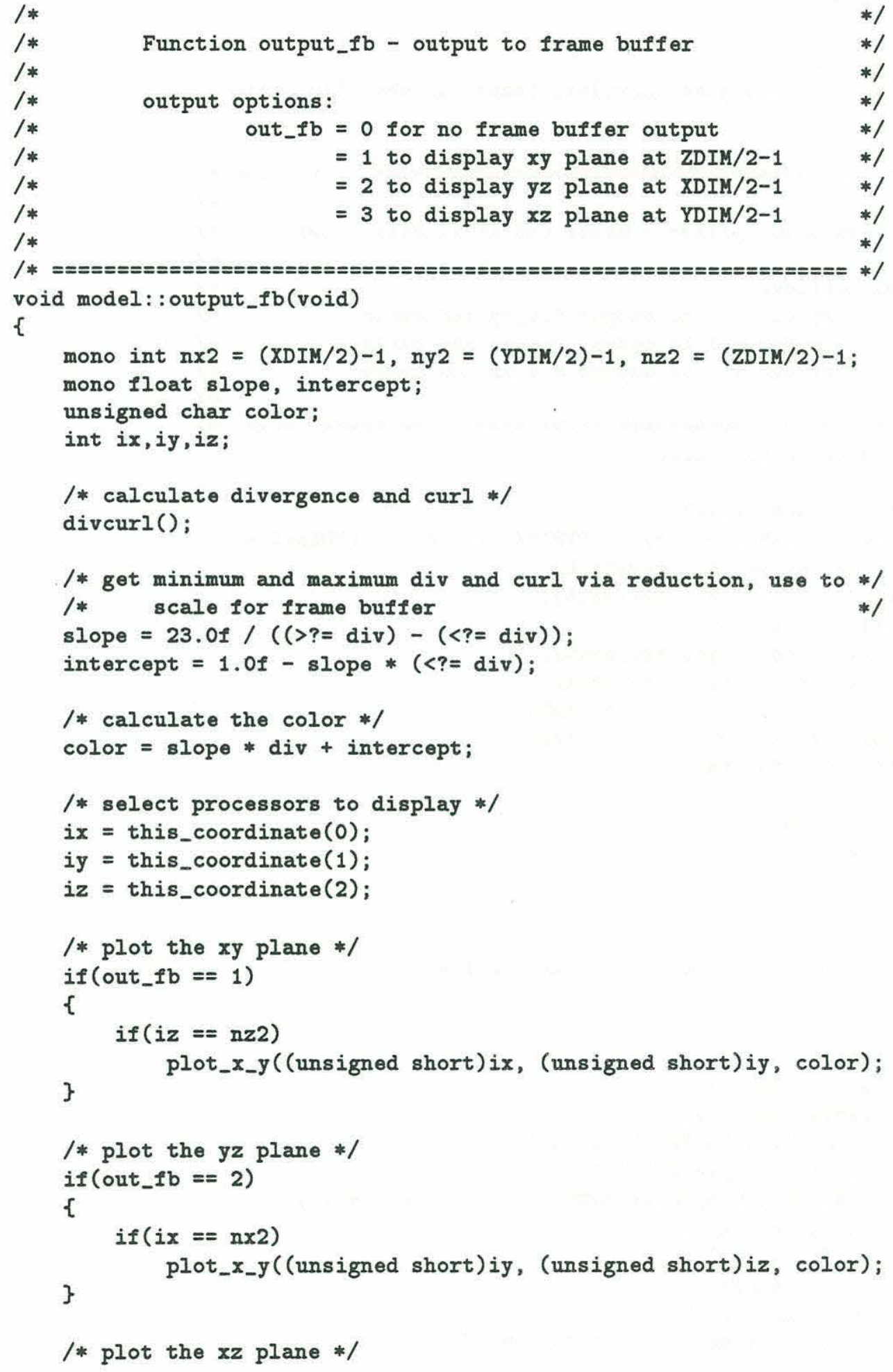




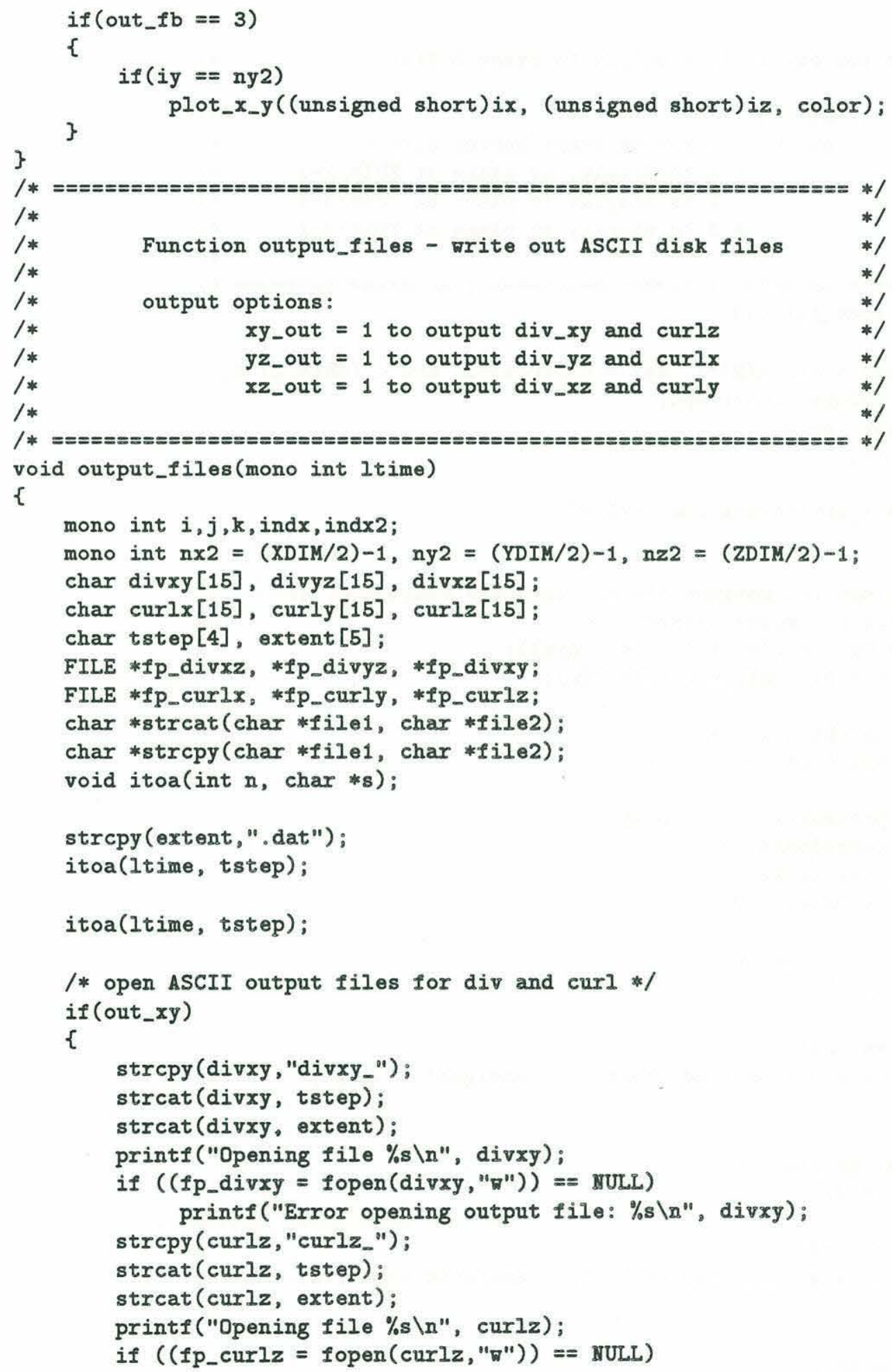




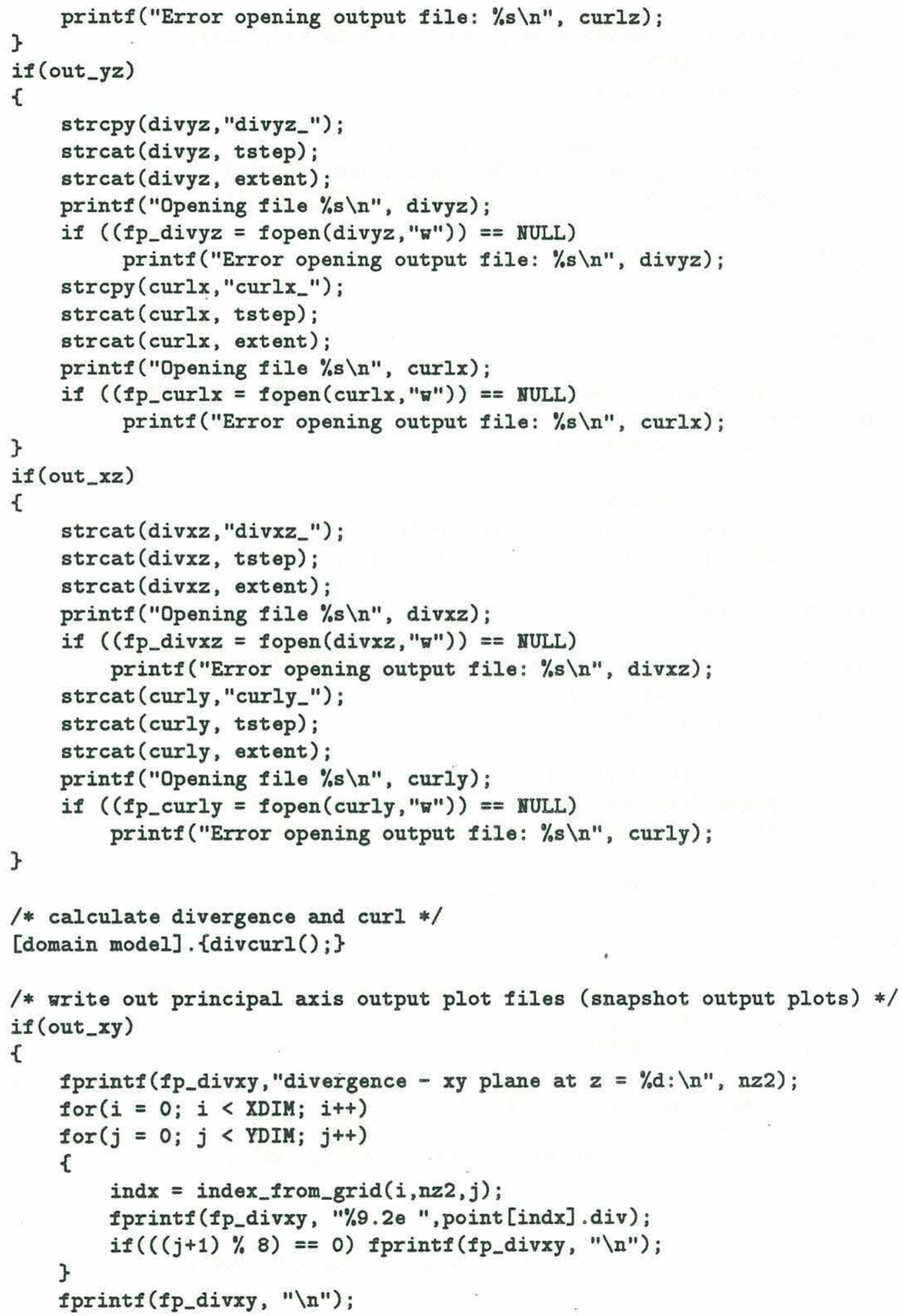




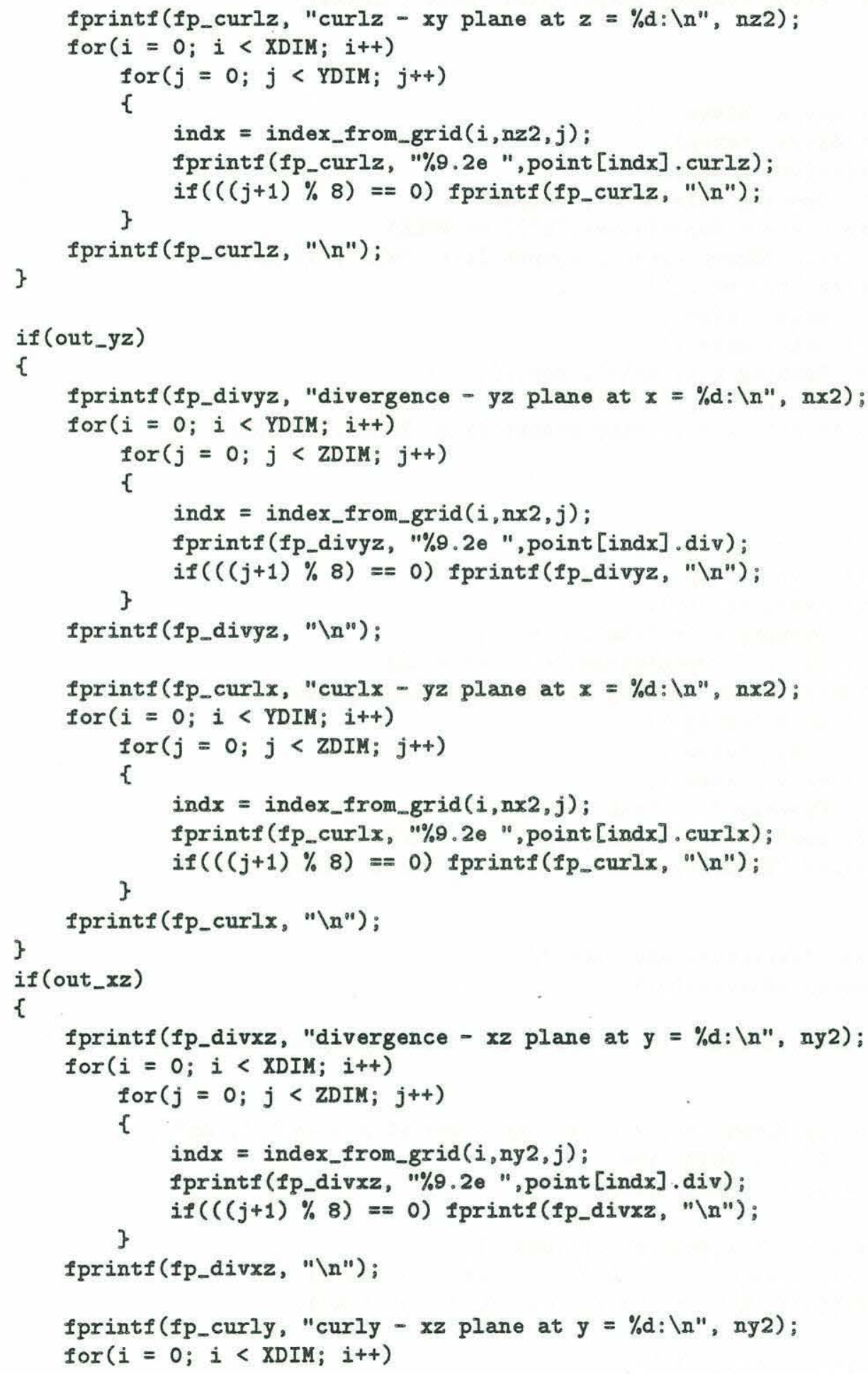




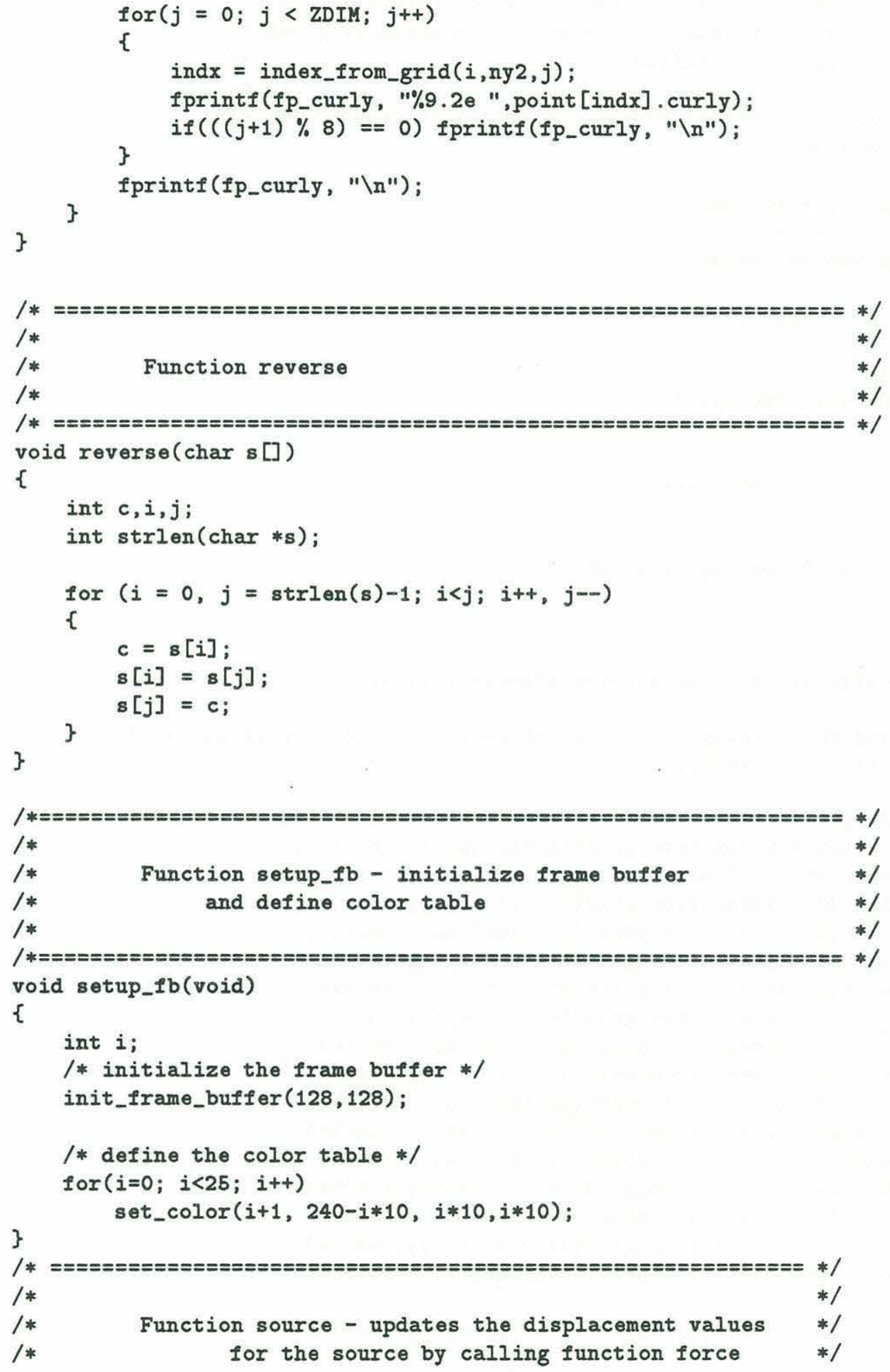




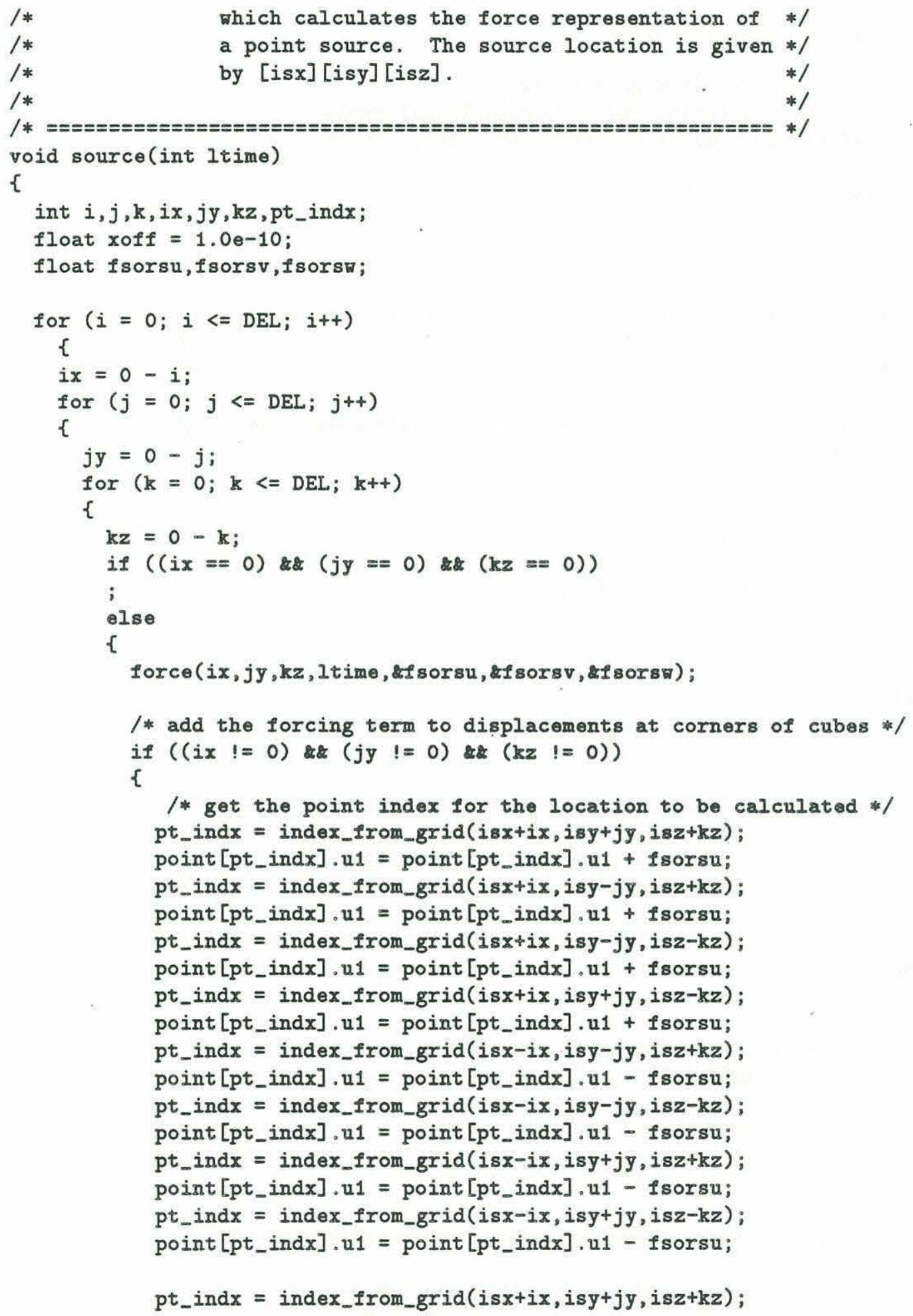




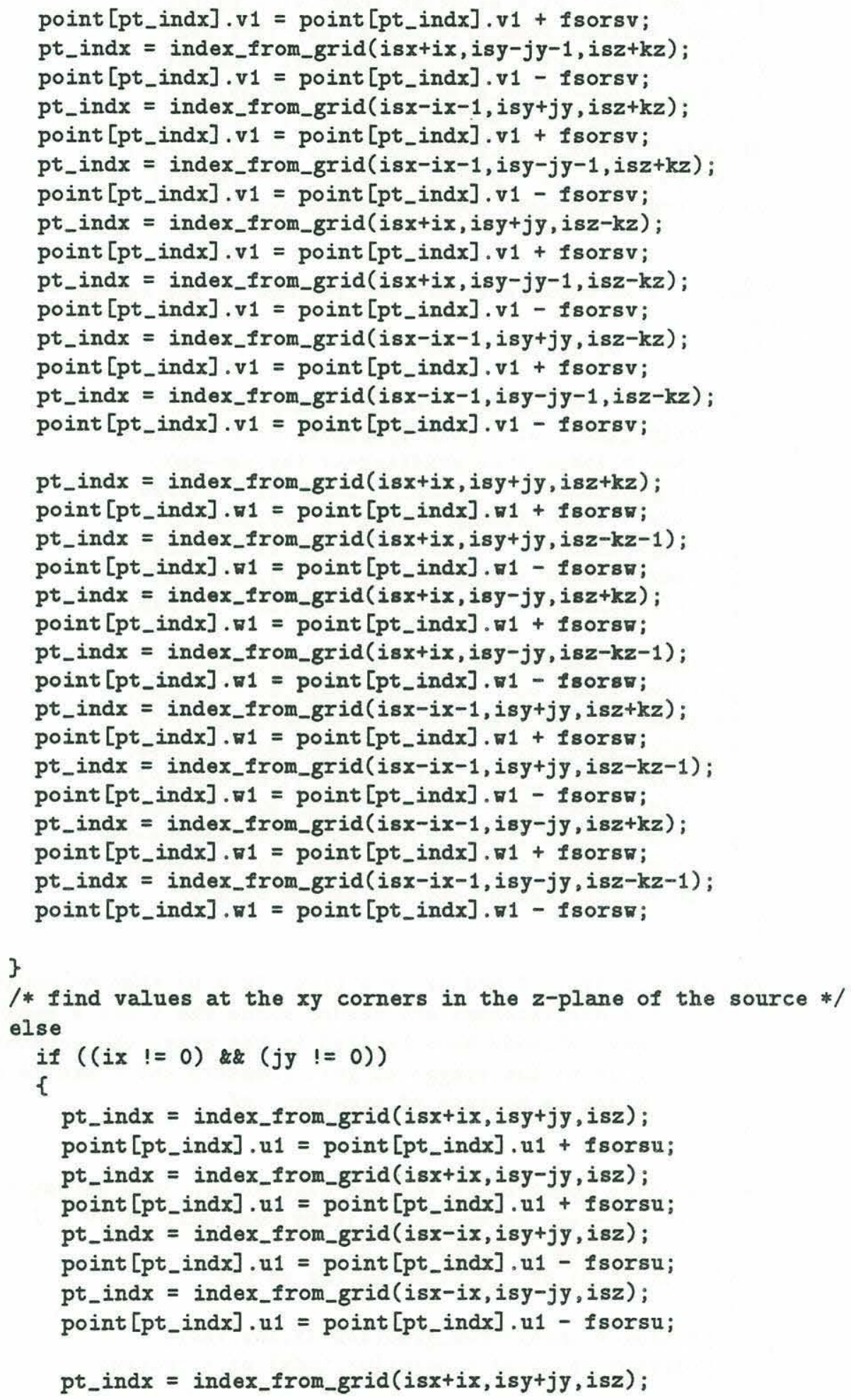




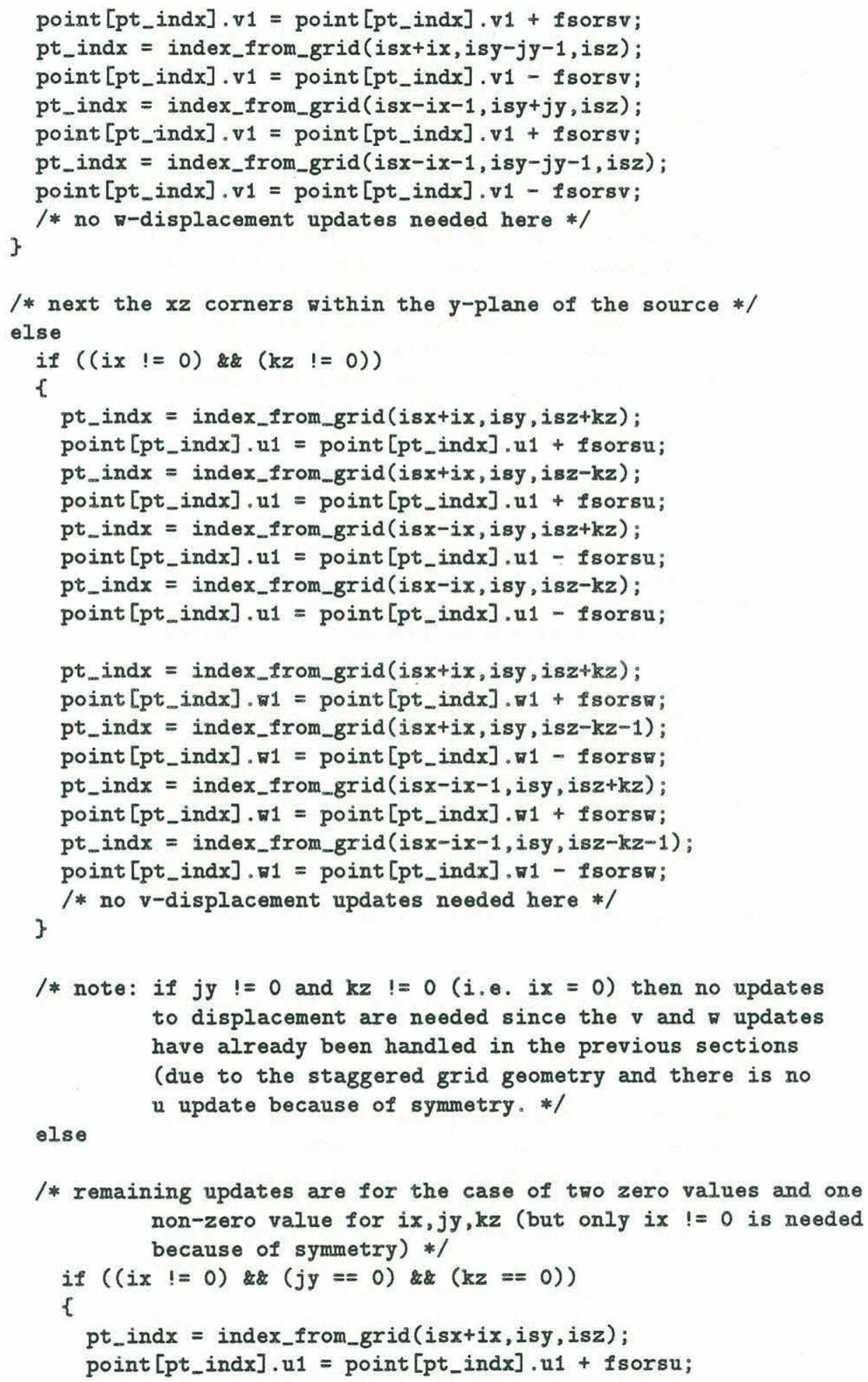




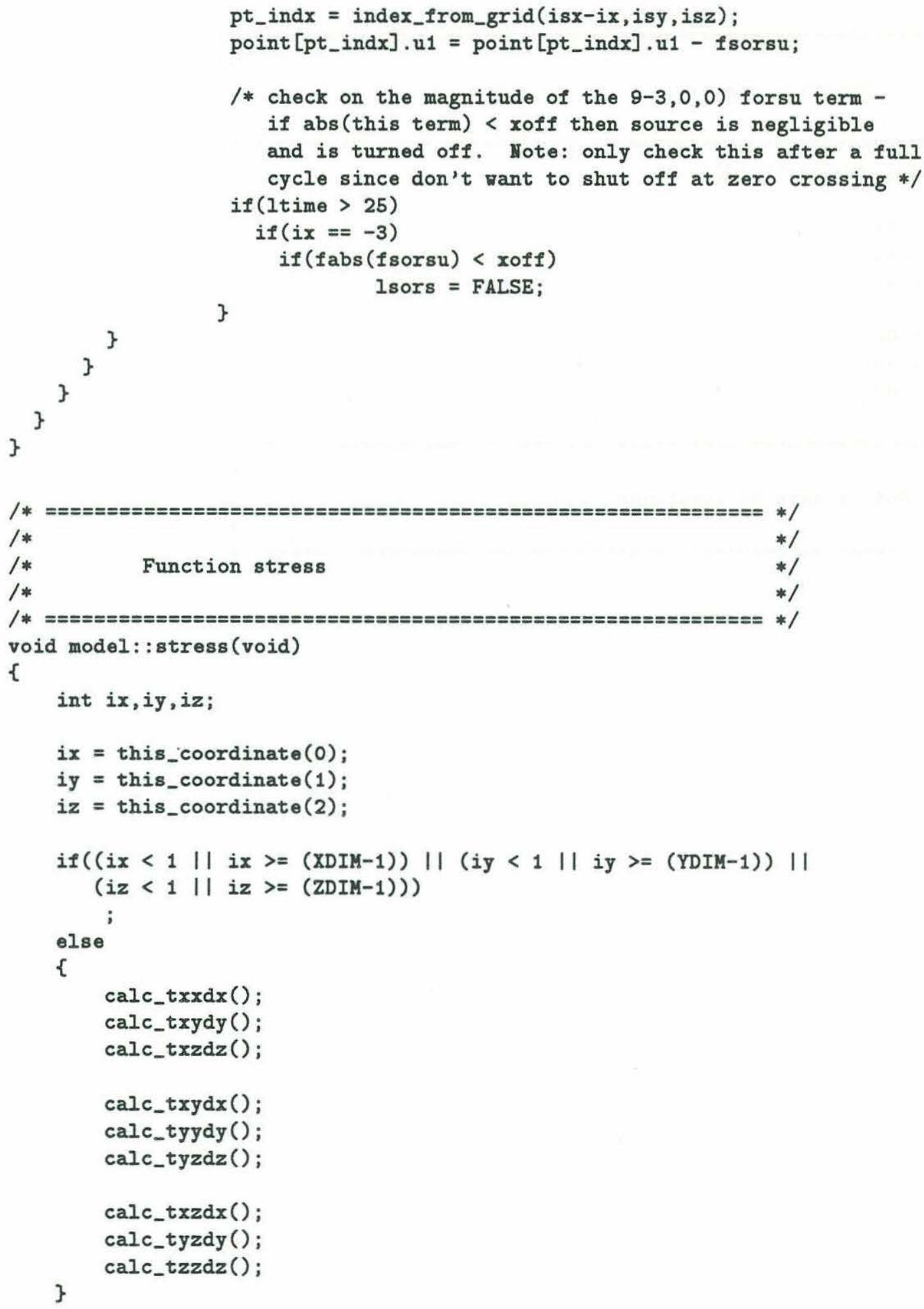




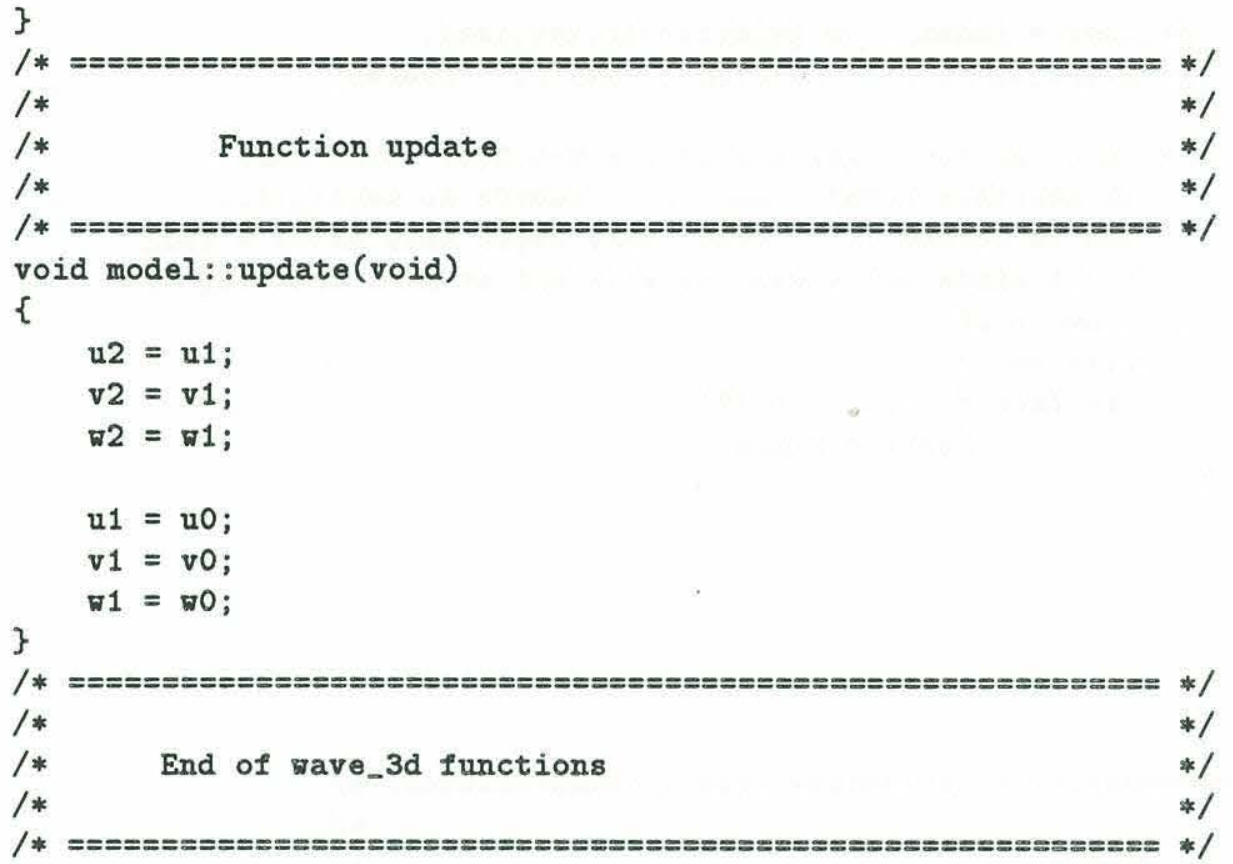




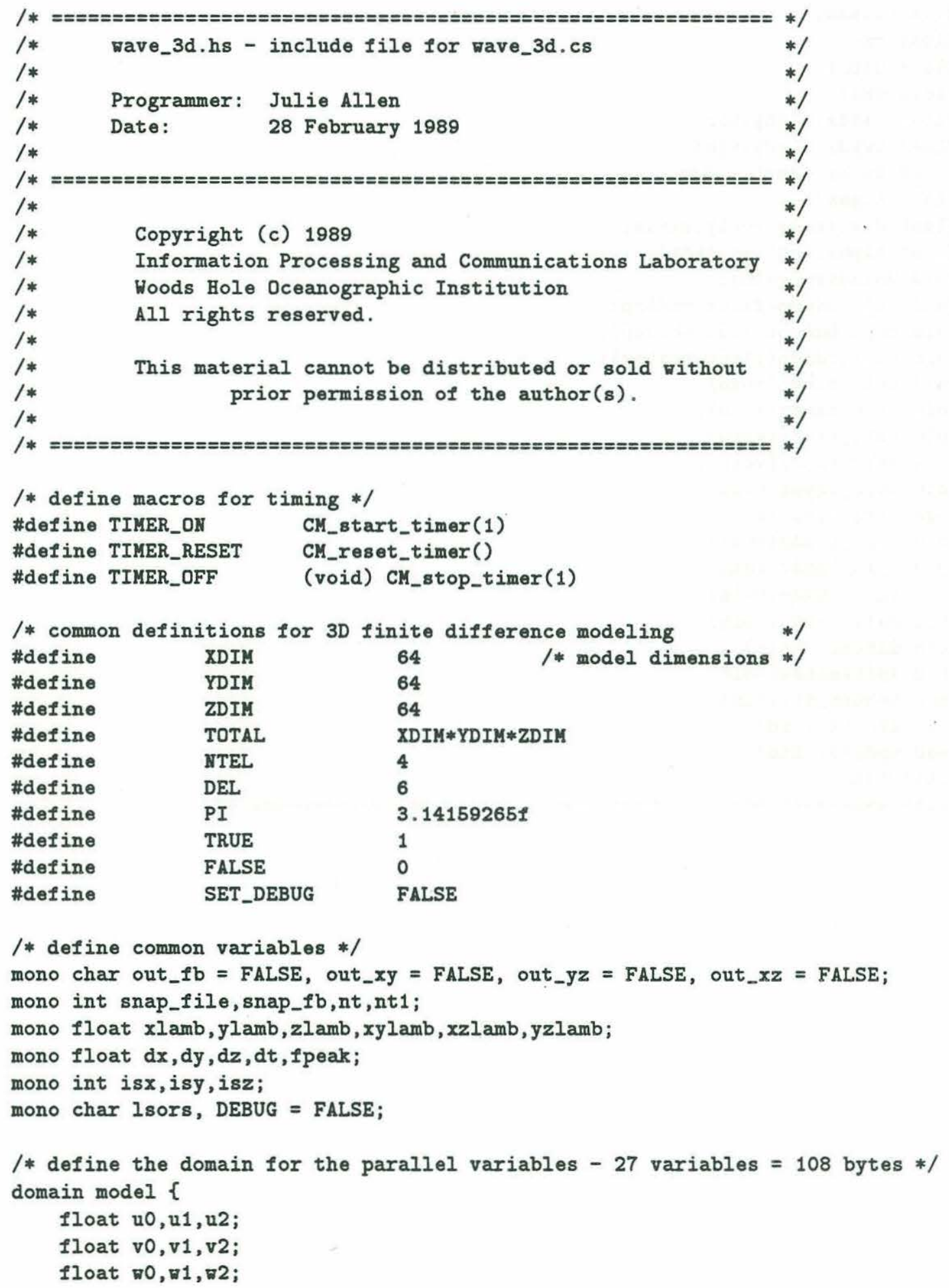




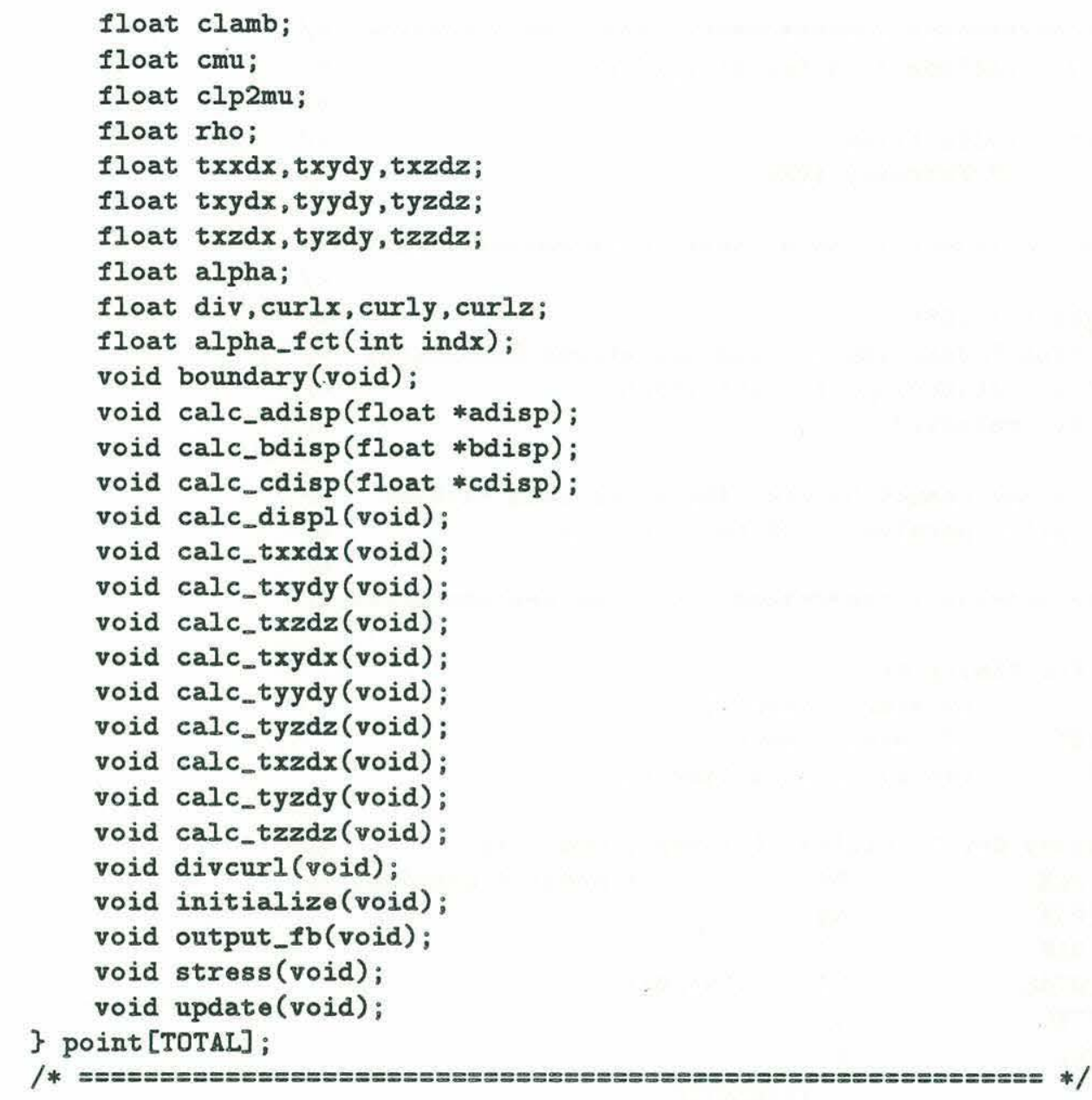




\section{D.2 convert program}

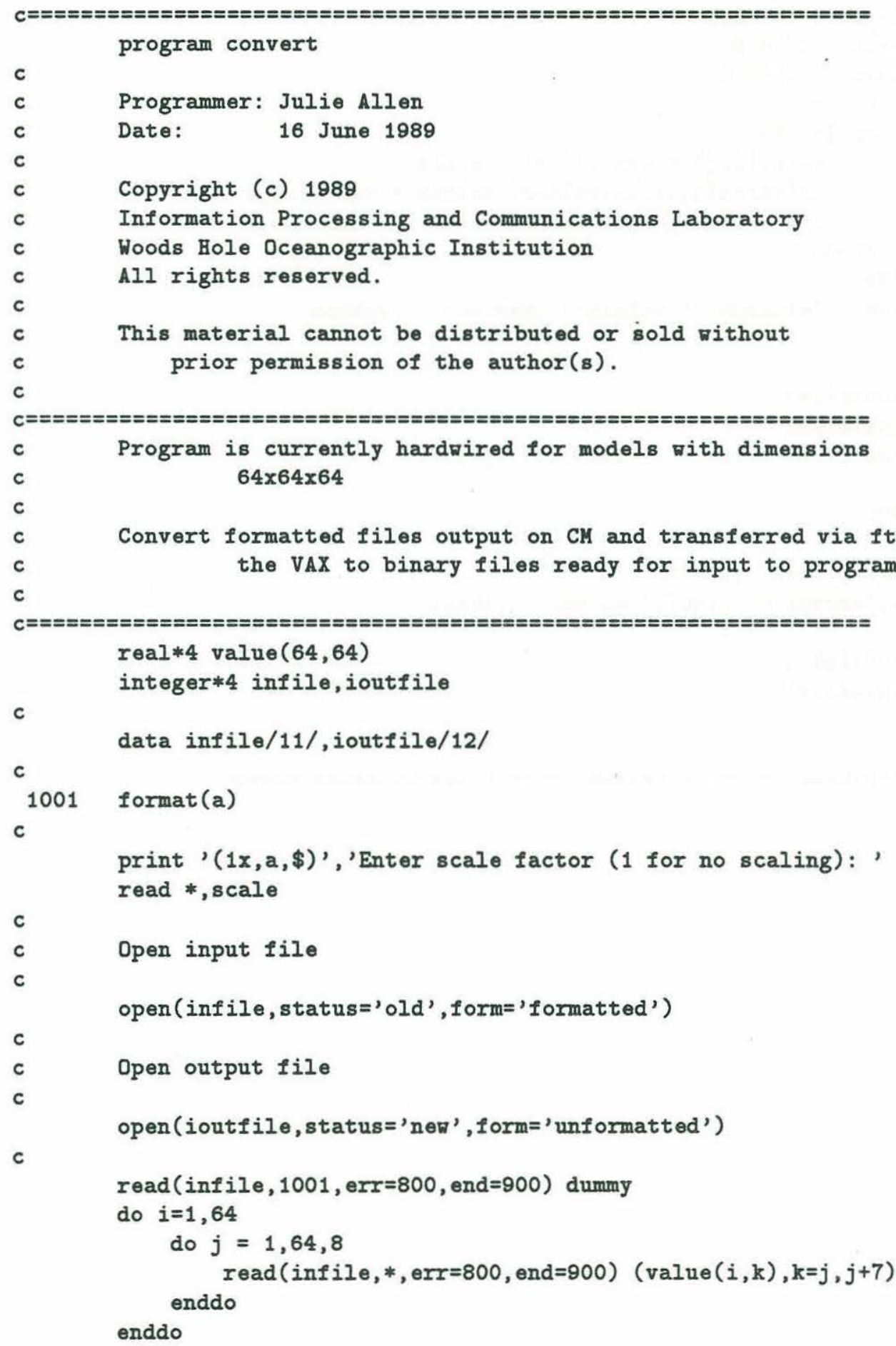




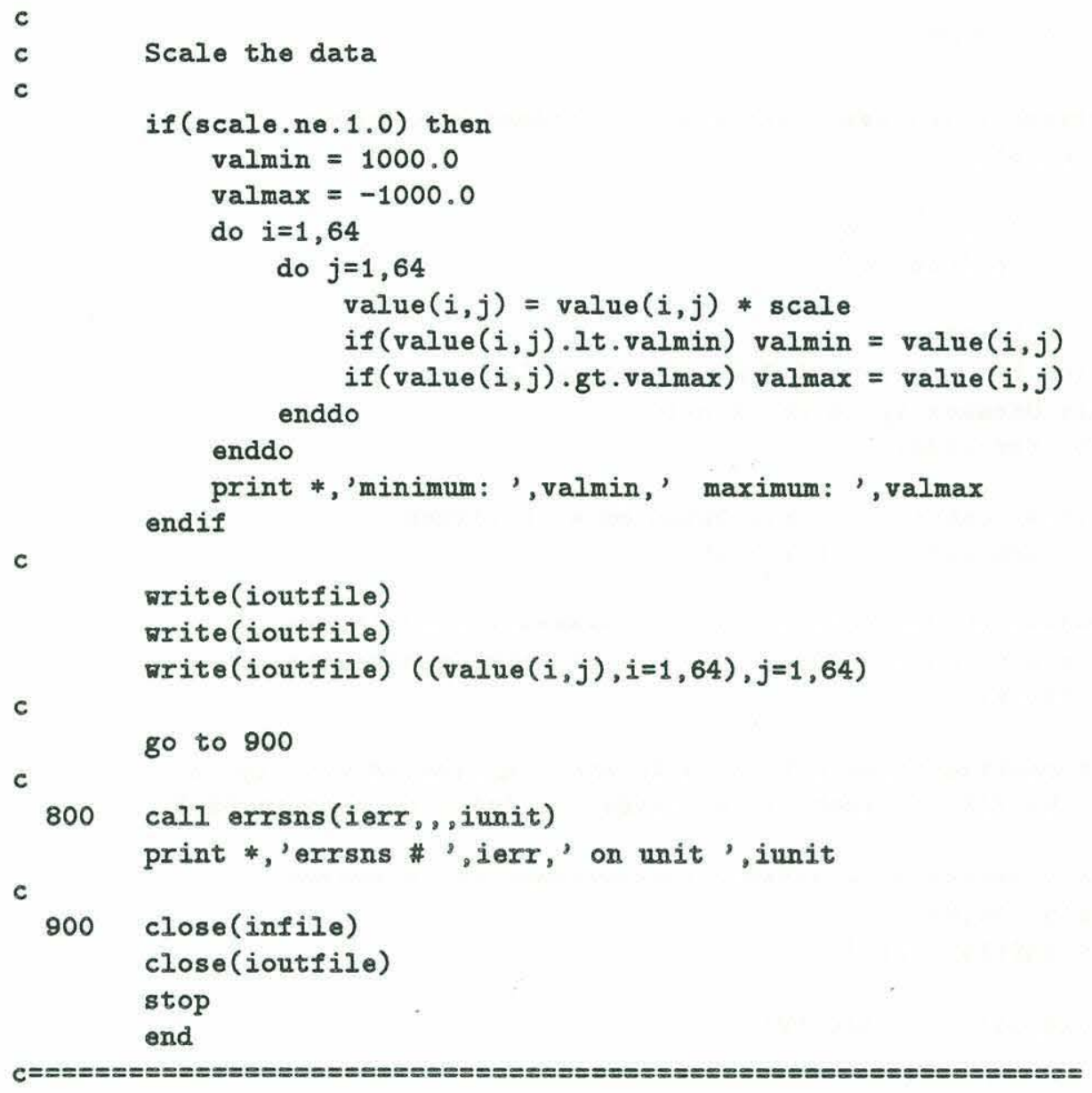




\section{D.3 n-dimensional grid library}

The following source code, used in the wave-3d program, was written by Robert Whaley, Site Representative from TMC at the NRL Connection Machine Facility.
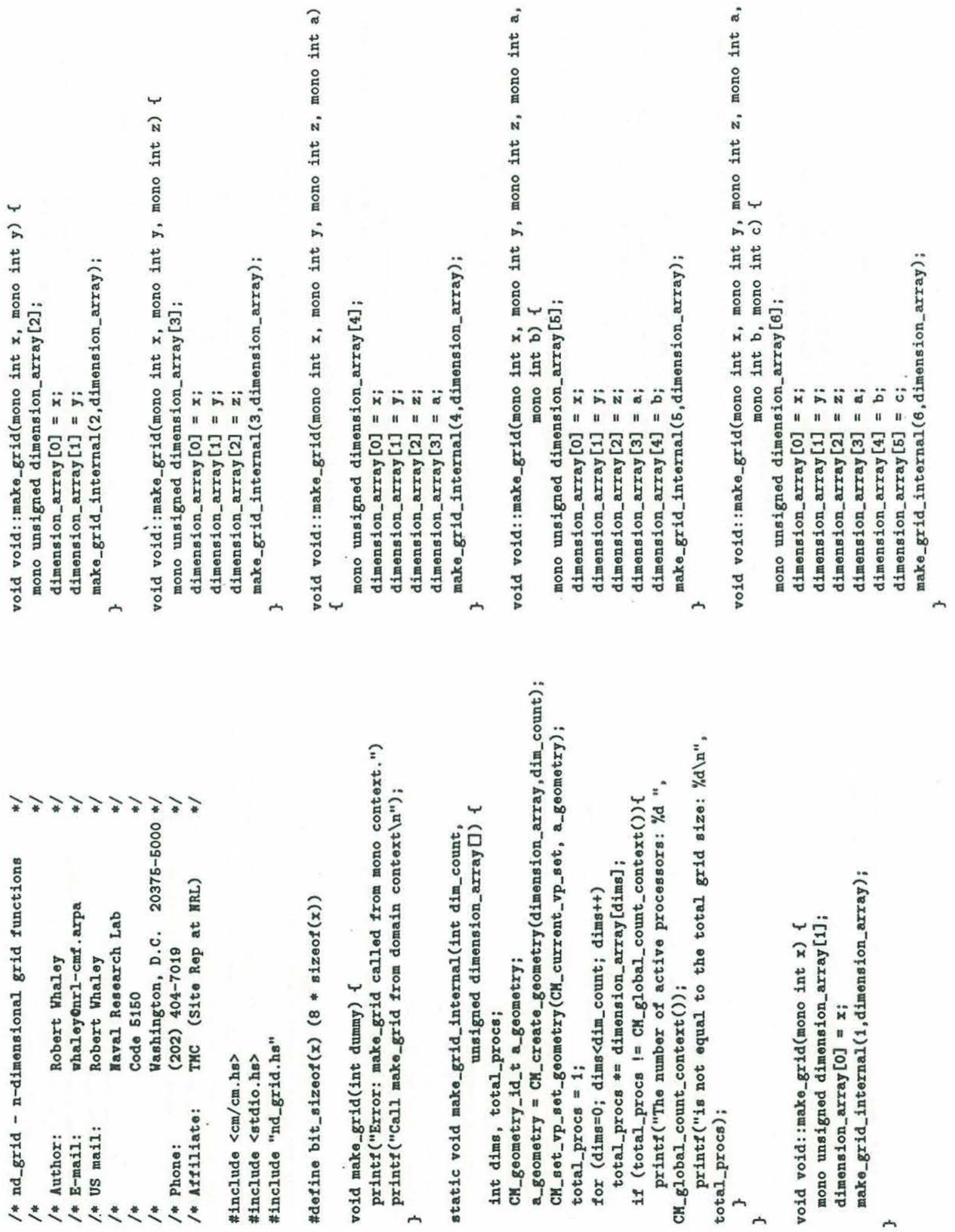

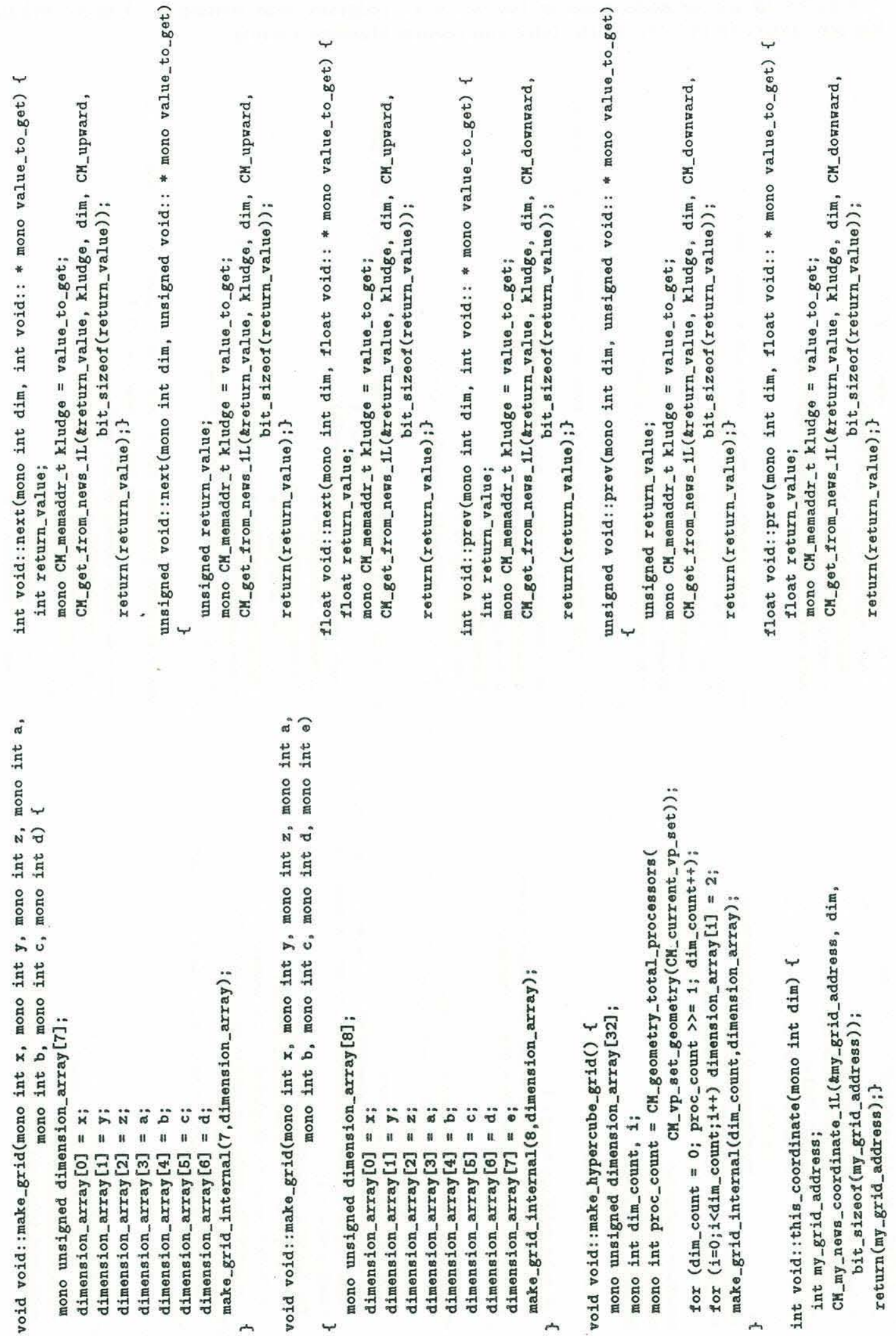

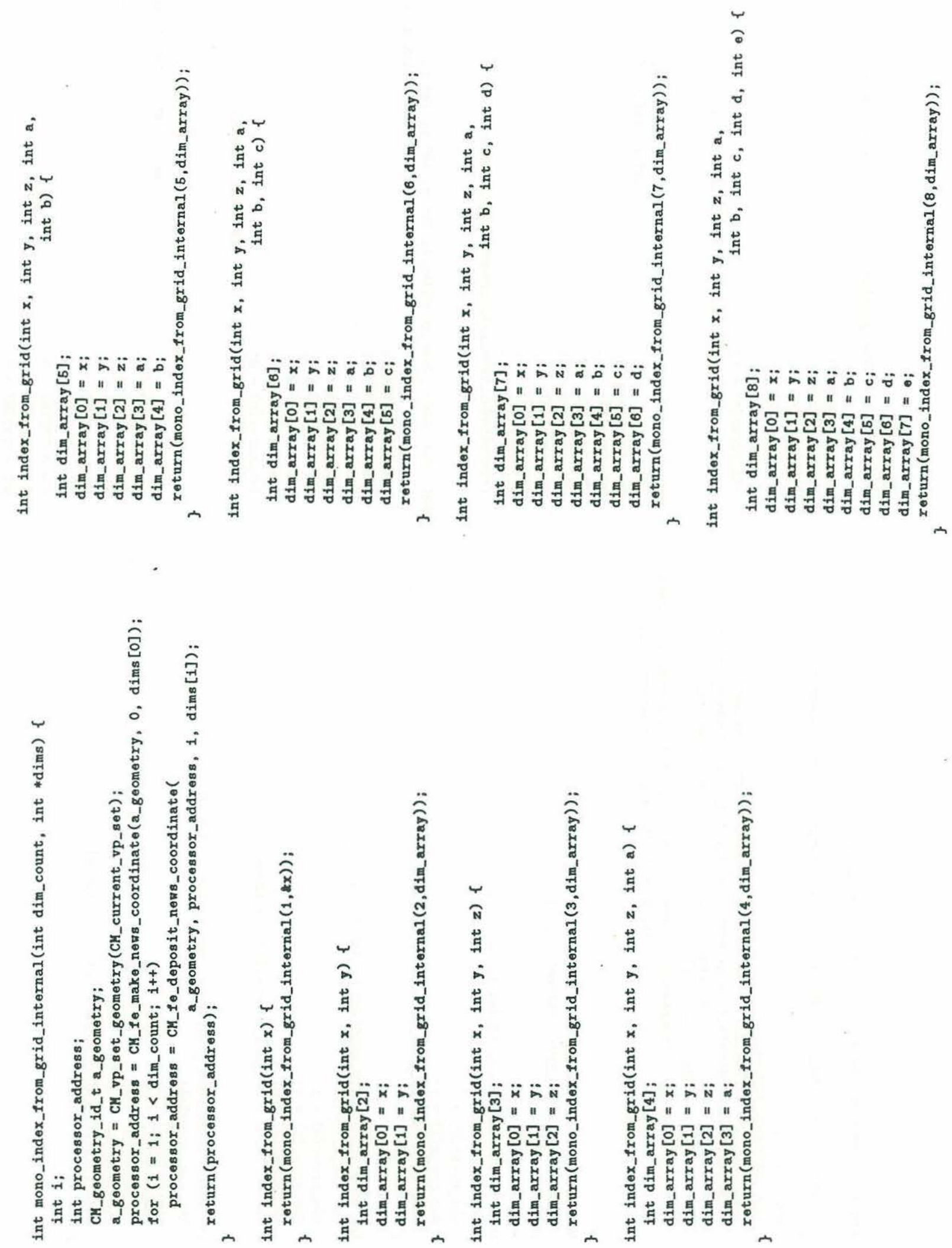

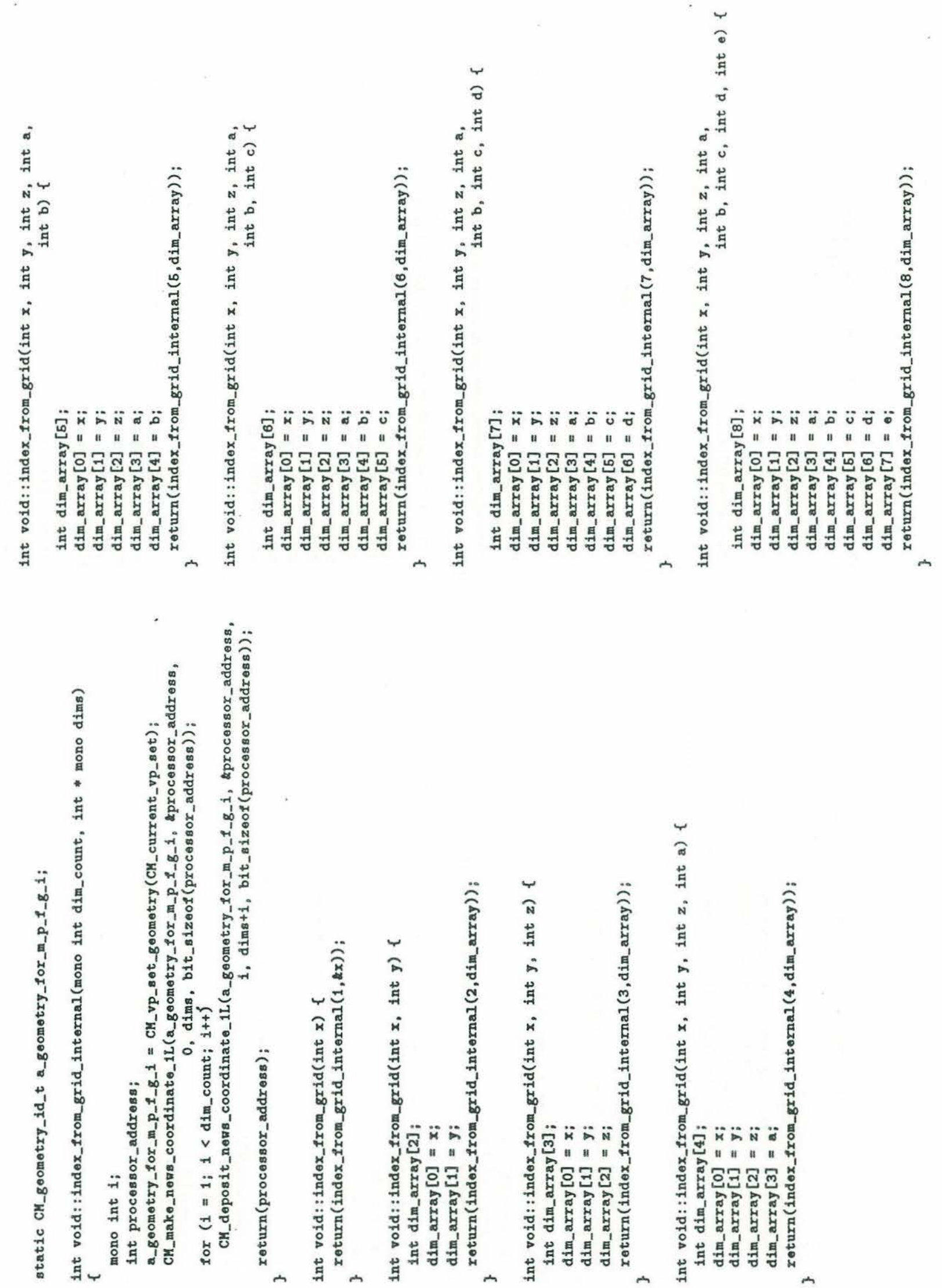

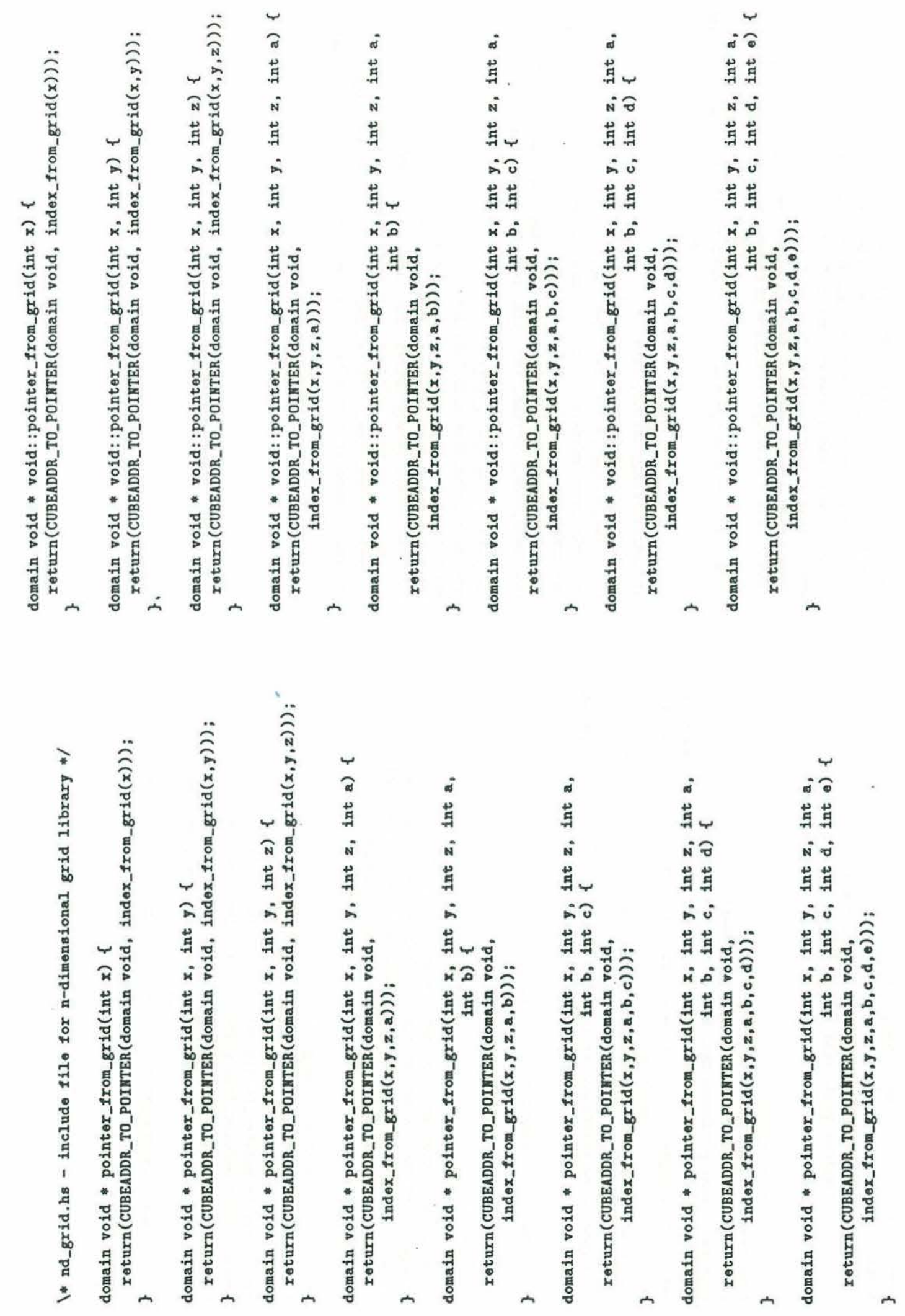

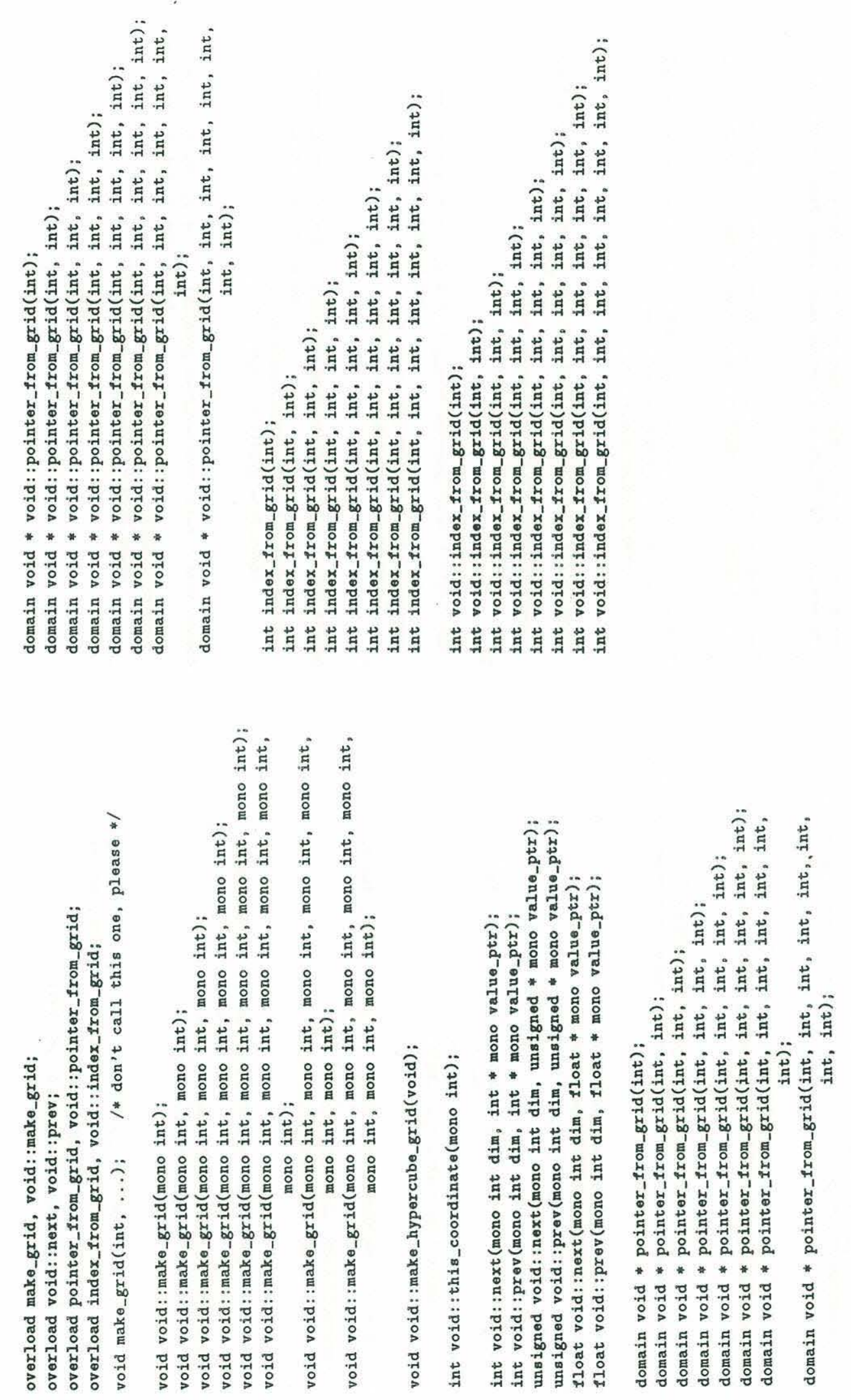


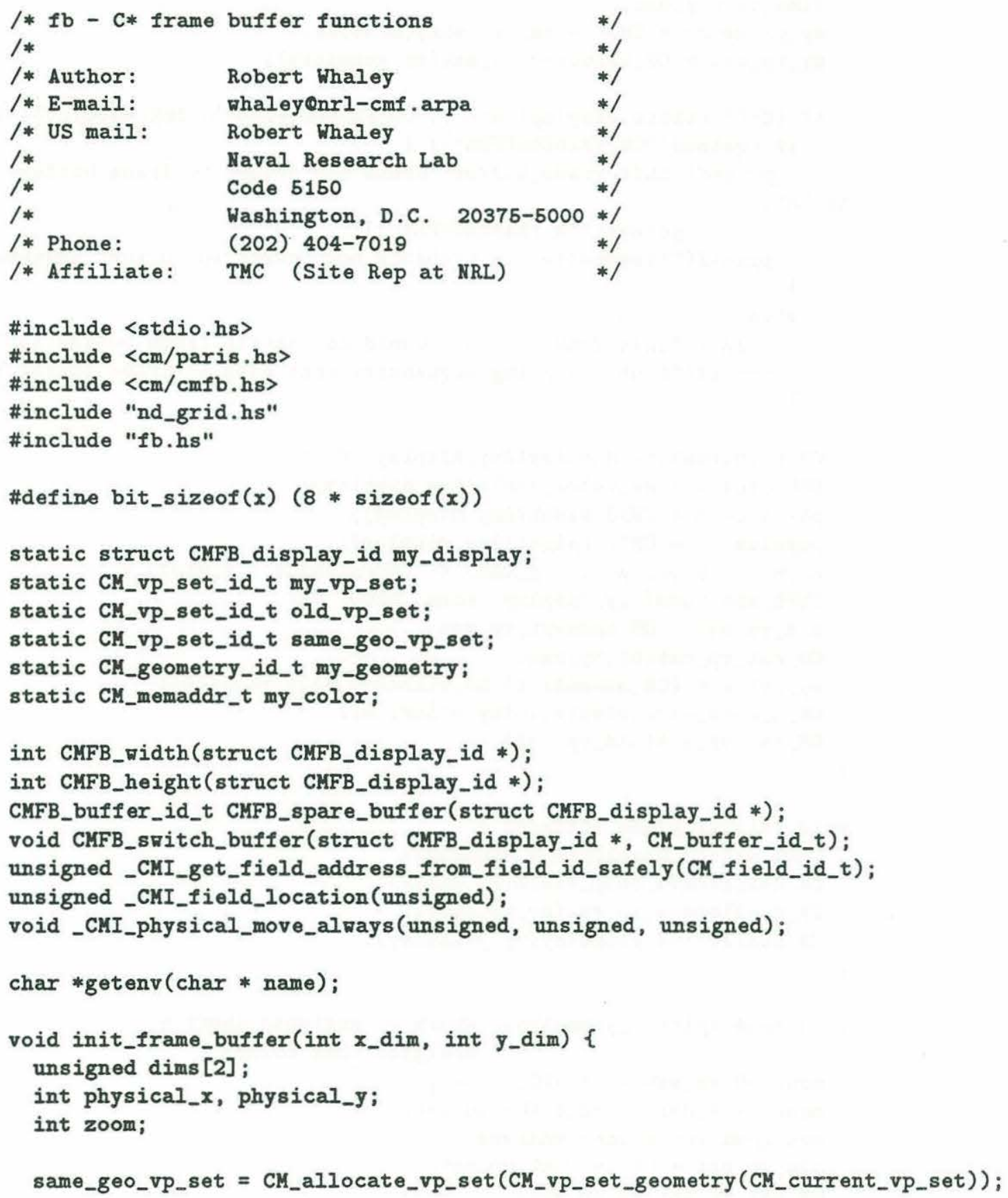




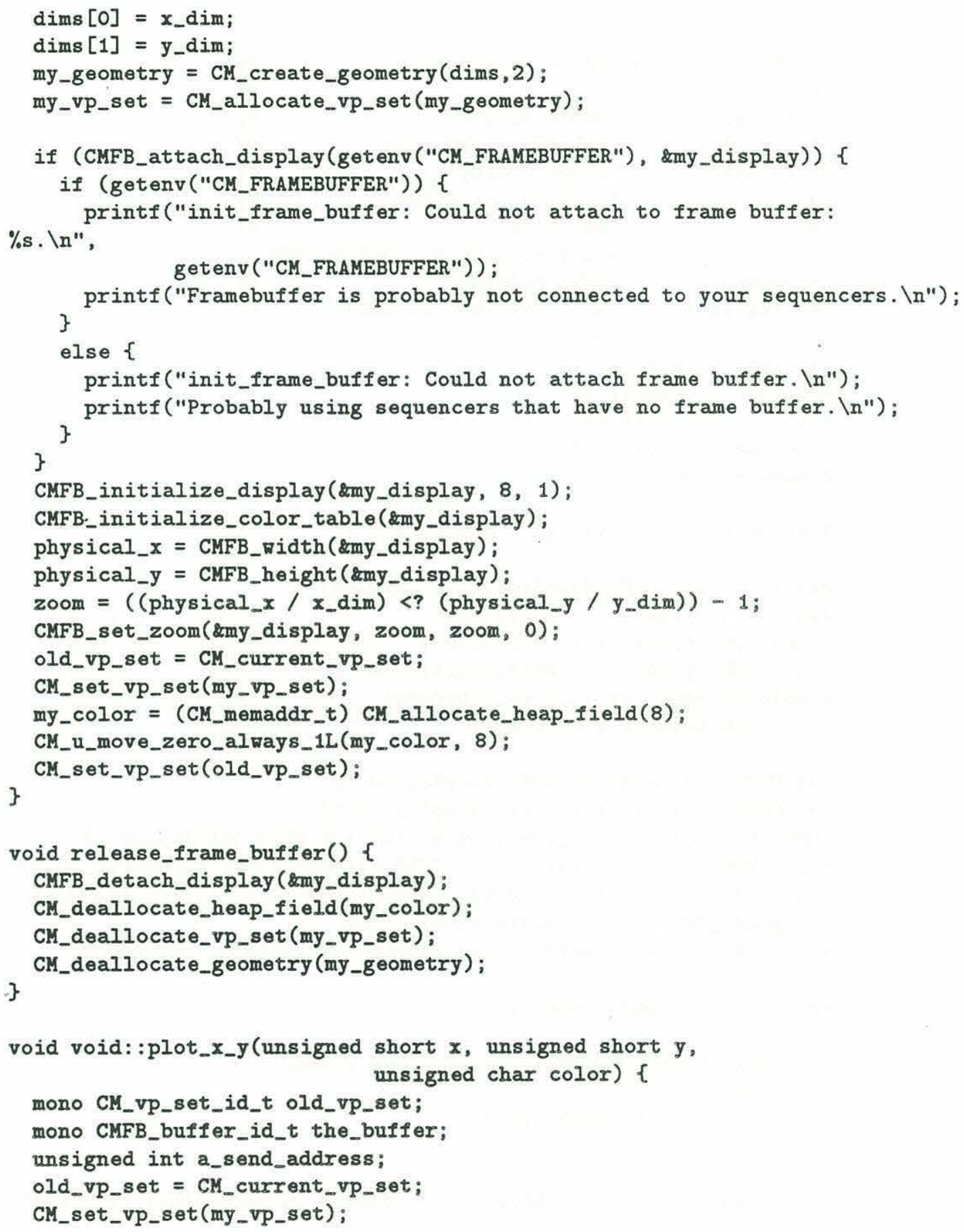




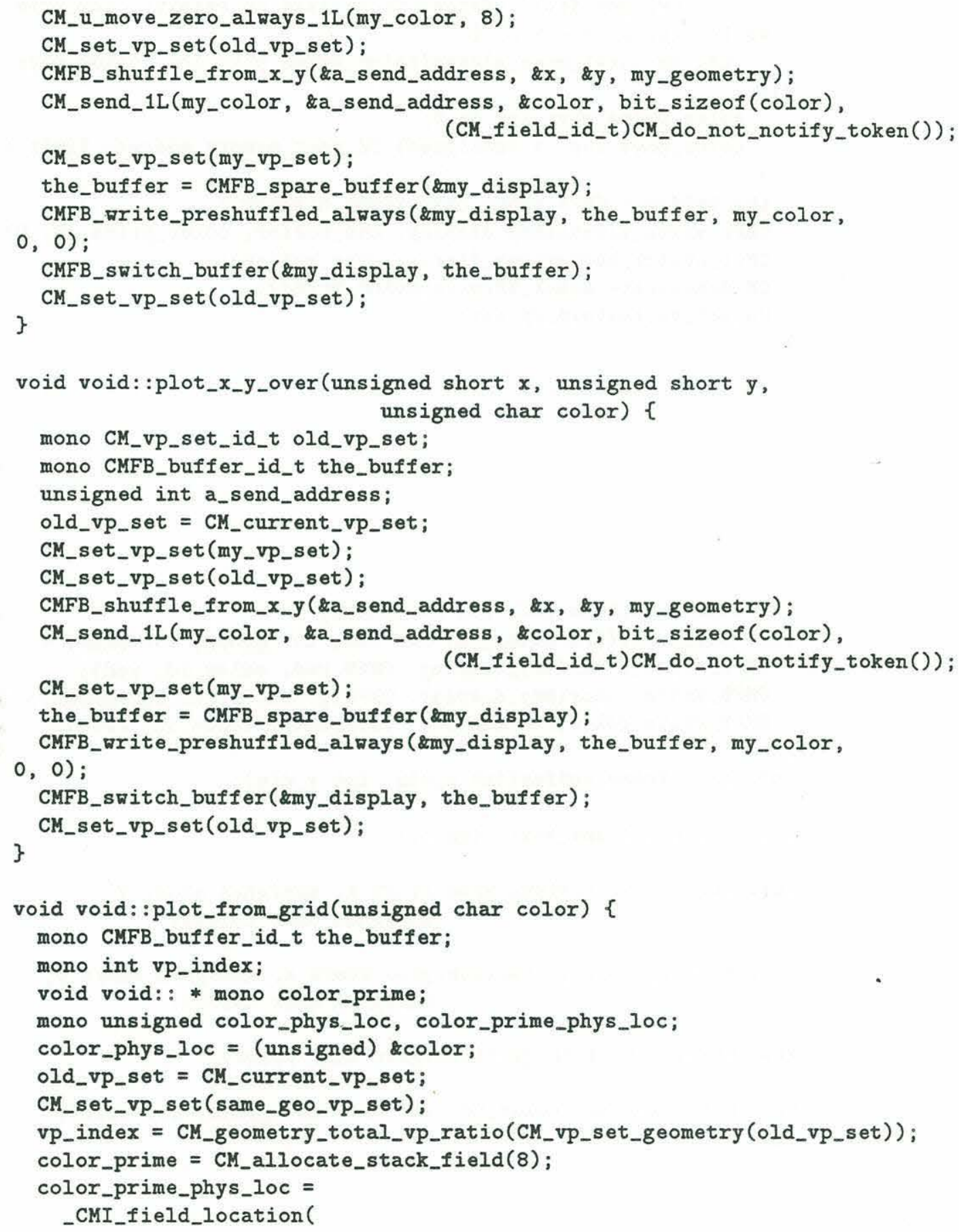




\section{References}

Alterman, Z. and F. C. Karal, 1968, Propagation of elastic waves in layered media by finite difference methods, BSSA, v. 58, 367-398.

Charrette, E. E., 1987, Three dimensional finite difference modeling on a very fine-grian parallel computer, in Annual Report of MIT Earth Resources Laboratore Reservoir Delineation Consortium.

Dougherty, Martin E. and Ralph A. Stephen, 1988, Seismic Energy Partitioning and Scattering in Laterally Heterogeneous Ocean Crust, PAGEOPH, Vol 128, 195-229.

Etgen, J. and K. Yomagida, 1988, Three dimensional wave propagation in the Los Angeles Basin, abstract, EOS, v. 69, no. 44, p 1325.

Fornberg, B., 1987, The pseudospectral method: comparisons with finite differences for the elastic wave equation, Geophysics, v. 41, p. 2-27.

Frankel, A. and R. W. Clayton, 1986, Finite difference simulations of seismic scattering: Implications for the propagation of short-period seismic waves in the crust and models of crustal heterogeneity, J. Geophys. Res. 91, 6465-6489.

Hillis, W. Daniel, 1987, The Connection Machine, Scientific American (256)6, 108-115.

Hunt, Mary M. and Ralph H. Stephen, 1986, A user's manual for finite difference synthetic seismogram codes on the CYBER 205 and CRAY XMP-12, W.H.O.I. Technical Memorandum No. 4-86.

Kelly, K. R., R. W. Ward, S. Treitel, and R. M. Alford, 1976, Synthetic seismograms: A finite difference approach, Geophysics 41, 2-27.

Levander, A. R., 1985, Use of the telegraphy equation to improve absorbing boundary efficiency for fourth-order acoustic wave finite difference schemes, Bull. Seism. Soc. Am. 75, 1847-1852.

Nicoletis, L., 1981, Simulation numerique del la propagation d'ondes sismiques dans les milieux stratifies a deux et trois dimensions: contributions a la construction et a l'interpretation des sismogrammes synthetiques, Ph.D. thesis, Universite Pierre et Marie Curie, Paris, France.

Stephen, R. A., 1983, A comparison of finite difference and reflectivity seismograms for marine models, Geophys. J. R. astr. Soc. 72, 39-58.

Stephen, R.A., 1984, Finite difference seismograms for laterally varying marine models, Geophys. J. R. astr. Soc., 79, 185-198.

Stephen, R. A., F. Pardo-Casas, and C. H. Cheng, 1985, Finite difference synthetic acoustic logs, Geophysics 50, 1588-1609.

Toksoz, M. N., A. M. Dainty and E. E. Charrette, 1988, Spatial variation of ground motion due to lateral heterogeneity, Proc. Internat. Workshop on Spatial variation of earthquake ground motion, in press.

Virieux, J., 1986, P-SV wave propagation in heterogeneous media: Velocity-stress finite difference method, Geophysics 51, 889-901. 


\section{DOCUMENTT LIBRARY}

July 5, 1989

Distribution List for Technical Report Exchange

Attn: Stella Sanchez-Wade

Documents Section

Scripps Institution of Oceanography

Library, Mail Code C-075C

La Jolla, CA 92093

Hancock Library of Biology \& Oceanography

Alan Hancock Laboratory

University of Southern California

University Park

Los Angeles, CA 90089-0371

Gifts \& Exchanges

Library

Bedford Institute of Oceanography

P.O. Box 1006

Dartmouth, NS, B2Y 4A2, CANADA

Office of the International

Ice Patrol

c/o Coast Guard R \& D Center

Avery Point

Groton, CT 06340

Library

Physical Oceanographic Laboratory

Nova University

8000 N. Ocean Drive

Dania, FL 33304

NOAA/EDIS Miami Library Center

4301 Rickenbacker Causeway

Miami, FL 33149

Library

Skidaway Institute of Oceanography

P.O. Box 13687

Savannah, GA 31416

Institute of Geophysics

University of Hawaii

Library Room 252

2525 Correa Road

Honolulu, HI 96822

Library

Chesapeake Bay Institute

4800 Atwell Road

Shady Side, MD 20876

MIT Libraries

Serial Journal Room 14E-210

Cambridge, MA 02139
Director, Ralph M. Parsons Laboratory Room 48-311

MIT

Cambridge, MA 02139

Marine Resources Information Center

Building E38-320

MIT

Cambridge, MA 02139

Library

Lamont-Doherty Geological Observatory

Colombia University

Palisades, NY 10964

Library

Serials Department

Oregon State University

Corvallis, OR 97331

Pell Marine Science Library

University of Rhode Island

Narragansett Bay Campus

Narragansett, RI 02882

Working Collection

Texas A\&M University

Dept. of Oceanography

College Station, TX 77843

Library

Virginia Institute of Marine Science

Gloucester Point, VA 23062

Fisheries-Oceanography Library

151 Oceanography Teaching Bldg.

University of Washington

Seattle, WA 98195

Library

R.S.M.A.S.

University of Miami

4600 Rickenbacker Causeway

Miami, FL 33149

Maury Oceanographic Library

Naval Oceanographic Office

Bay St. Louis

NSTL, MS 39522-5001

Marine Sciences Collection

Mayaguez Campus Library

University of Puerto Rico

Mayagues, Puerto Rico 00708 


\begin{tabular}{|c|c|c|c|}
\hline $\begin{array}{l}\text { REPORT DOCUMENTATION } \\
\text { PAGE }\end{array}$ & $\begin{array}{l}\text { 1. REPORT NO. } \\
\text { WHOI-89-48 }\end{array}$ & 2. & 3. Recipient's Accession No. \\
\hline \multirow{2}{*}{\multicolumn{3}{|c|}{$\begin{array}{l}\text { 4. Title and Subtitle } \\
\text { Calculation of 3-Dimensional Synthetic Seismograms on the Connection Machine }\end{array}$}} & $\begin{array}{l}\text { 5. Report Date } \\
\text { October, } 1989\end{array}$ \\
\hline & & & 6. \\
\hline \multicolumn{3}{|l|}{$\begin{array}{l}\text { 7. Author(s) } \\
\text { J.M. Allen and D.R. Burns }\end{array}$} & $\begin{array}{l}\text { 8. Performing Organization Rept. No. } \\
\text { WHOI-89-48 }\end{array}$ \\
\hline \multicolumn{3}{|c|}{ 9. Performing Organization Name and Address } & 10. Project/TaskWWork Unit No. \\
\hline \multicolumn{3}{|c|}{$\begin{array}{l}\text { The Woods Hole Oceanographic Institution } \\
\text { Woods Hole, Massachusetts } 02543\end{array}$} & $\begin{array}{l}\text { 11. Contract(C) or Grant(G) No. } \\
\text { (C) N00014-87-K-0007 } \\
\text { (G) N00014-89-J-1012 }\end{array}$ \\
\hline \multicolumn{3}{|c|}{ 12. Sponsoring Organization Name and Address } & $\begin{array}{l}\text { 13. Type of Report \& Period Covered } \\
\text { Technical Report }\end{array}$ \\
\hline \multicolumn{3}{|c|}{ Funding was provided by the Office of Naval Research } & 14. \\
\hline
\end{tabular}

\section{Supplementary Notes}

This report should be cited as: Woods Hole Oceanog. Inst. Tech. Rept., WHOI-89-48

\section{Abstract (Limit: 200 words)}

A three dimensional, second order finite difference method was used to create synthetic seismograms for elastic wave propagation in heterogeneous media. These synthetic seismograms are used to model rough seafloor, the shallow crust, or complex structural and stratigraphic settings with strong lateral heterogeneities. The finite difference method is preferred because it allows models of any complexity to be generated and includes all multiple scattering, wave conversion and diffraction effects. The method uses a fully staggered grid as developed by Virieux (1986). Wavefront snapshots and time series output allow the scattering and focussing of different wave modes with direction to be visualized.

The extensive calculations required for realistic size models stretches the resources of serial computers like the VAX 8800 . On the Connection Machine, a massively parallel computer, the finite difference grid can be directly mapped onto the virtual processors, reducing the nested time and space loops in the serial code to a single time loop. As a result, the computation time is reduced dramatically.

\section{Document Analysis a. Descriptors}

1. 3-D synthetic seismograms

2. parallel computing

3. acoustic modeling

b. Identifiers/Open-Ended Terms

c. COSATI Field/Group

18. Availability Statement

Approved for publication; distribution unlimited.

\begin{tabular}{|l|c|}
$\begin{array}{c}\text { 19. Security Class (This Report) } \\
\text { UNCLASSIFIED }\end{array}$ & $\begin{array}{c}\text { 21. No. of Pages } \\
79\end{array}$ \\
\hline 20. Security Class (This Page) & 22. Price \\
\hline
\end{tabular}

